\title{
ANALYTICAL FOUNDATIONS OF THE THEORY OF QUASICONFORMAL MAPPINGS IN $R^{n}$
}

\author{
B. BOJARSKI and T. IWANIEC
}

This paper gives an exposition of basic analytical properties of quasiconformal (and quasiregular) mappings of $n$-dimensional domains. It grew out as a byproduct and a proper part of our work on the monograph [10] on analytic methods in the quasiconformal theory. This comes out of a general programme of studying the problems of $n$-dimensional quasiconformal mappings exposing and exploiting as much as possible the interconnections between the quasiconformal mappings and various problems of partial differential equations, differential geometry and classical analysis. This programme outlined in [6], [7], and essentially extended in our plenary lecture [8] at the Conference on "Global Analysis and Differential Geometry" in Garwitz, DDR, October 1981, was based on the opinion that the natural interrelations between the quasiconformal theory in $n \geqq 3$ dimensions and partial differential equations, in contrast to the case $n=2$, have not been sufficiently explored. The development of the two-dimensional theory indicated rather clearly that the methods of p.d.e. supplied the most flexible and universal tools for the study of quasiconformal problems [5]. However, the study of these relations in the $n \geqq 3$ dimensional case requires a much broader line of research than the case $n=2$. This can be seen in relatively recent papers of L. Ahlfors [1], in the work of Ju. Rešetnjak during the last 15 years, e.g. [29], [30], [31],, in the recent papers of the Finnish School, especially of Martio, Granlund and Lindqvist [16], as well as in our papers [7], [9], [20].

It is our belief that the exposition of analytical methods, especially those connected with partial differential equations, helps to see in the proper perspective the deep analogies and links as well as the differences between the two-dimensional and $n$-dimensional quasiconformal theory.

In the recent literature on quasiconformal and quasiregular theory a variety of methods has been applied. Of these the most effective proved to be the methods connected with the notion of the modulus of a path family, which were essential in obtaining the spectacular results on extending the Ahlfors-Nevanlinna theory to $n$-dimensions [32], In our presentation of the study of foundations of the quasiregular theory this method is deliberately avoided. This should be by no means understood as a sign of our tending to underestimate the value of the method. 
However, it seems that the prevalence of the modulus method in the current research papers on quasiregular and quasiconformal mappings problems is probably responsible, in a sense, for some kind of isolation of the quasiconformal theory in $R^{n}$ from other branches of analysis, whereas we think that the true value of the theory lies in its interdisciplinary role. The two-dimensional case can be illustrated by [3], [4], [5], [23], [24] and many other papers; the view that this is the case also in dimensions greater than two was underlying the main ideas in [6].

In the process of the work on various aspects of quasiconformal theory in several variables an urgent need for a methodologically homogeneous and self-contained presentation of the "well-known" fundamental facts of the theory was coming up again and again. We included it in the first chapters of [10]. However, when these were essentially ready and the work on the whole of [10] was naturally delayed, for various reasons, we thought it might be useful to prepare this part of [10] in the form of a completely self-contained, introductory publication. Therefore we restrict our discussion here to the study of basic local analytic properties of quasiregular mappings, including their behaviour in the simplest formulas of differential and integral calculus. Only in the last few pages we touch some geometric problems, just to show to a non-specialist that the analytical tools developed are sufficient for the full proof of some important geometric properties of quasiregular mappings.

As already said, we tried to make the presentation direct and self-contained to the extent needed for [10]. Naturally, at several essential points, the ideas used have their roots in the existing literature on quasiconformal mappings, especially in the papers of Ju. Rešetnjak and the Finnish School. However, except the Sobolev local imbedding inequality and some standard analysis, all other facts are proved explicitly. In our consideration the essential step in the basic fact that the conditions (2.2a) and (2.2b) imply $f \in W_{n+\varepsilon}^{1}\left(\Omega^{\prime}\right)$ for every open subdomain $\Omega^{\prime} \subset \subset \Omega$ and some positive $\varepsilon$. This first step improving the a priori assumed regularity of q.r. mappings stated in 2a), as was shown in [5] and [40] for the case of two dimensions, opens the most direct way for the study of general analytical properties of q.r. mappings. Theorem 5.1 for locally quasiconformal mappings, $n \geqq 3$, was proved by F. Gehring in [14] (see also [25] for q.r. maps), relying on the modulus method of families of curves, which, traditionally, is applied to continuous mappings. The general case for q.r. maps follows also from the result for variational inequalities by Elcrat and Meyers [12]. The proof of Theorem 5.1 as presented here follows some ideas of T. Iwaniec first stated in [19] and developed later in [21], [22].

Since this paper is understood as a proper part of [10] or as a supplement to the existing literature on q.r.m. [16], [26-32], we do not give here any examples. Also, we do not try to discuss all consequences of our approach to quasiconformal theory. For this the reader is referred to [10]. Neither do we try to show the relevance of this presentation to the related parts of the theory of variational inequalities or problems in pure partial differential equations. This should be evident to the specialist. Quite a few examples will be given in [10]. 
Finally let us make some remarks referring to a programme of further research: We tried here to collect all relevant statements about general basic analytic properties of q.r. and q.c. maps in their most general formulation available at present. However, it is our opinion that most of these statements have not, as yet, got their final form; thus we think that such questions as the differentiability of q.r. maps, the conditions $\mathcal{N}$ and $\mathscr{N}^{-1}$, and the description of the set of zeros of the Jacobian $J_{f}$, have not been discussed in their natural set-up. As a matter of fact this natural set-up has in most cases not been found yet. And although the present general form of many facts about analytical properties of q.c. maps is sufficient for the basic constructions and analytical manipulations involved in the application of the theory, there is much left to be done in the study of the fundamental local problems of the theory. An interesting area of research in this connection lies, in our opinion, in the applications of the theory of "higher" variations of real valued functions as developed by Vituškin [41] and others [18]. However, we think that the study of the foundations of quasiconformal theory along the ideas of the theory of functions of real variables should proceed in balance with the investigations of the interaction of general quasiconformal theory with p.d.e's, variational inequalities, differential geometry and analysis in general. At this moment the latter directions of research are perhaps much more important than the former ones.

Let us finally mention that our references to the existing literature are far from being complete. A more exhaustive list will be given in [10].

\section{PRELIMINARIES AND NOTATION}

In this section we collect some basic facts related to the Lebesgue integration, Sobolev spaces and algebraic properties of the Jacobians. The material is essentially well-known though at some point we present the results in a novel way involving some modifications rather important for the applications in what follows.

1.1. Cube coverings and decompositions in $\boldsymbol{R}^{n} \cdot \boldsymbol{R}^{\boldsymbol{n}}$ denotes a Euclidean $\boldsymbol{n}$-space, with points $x=\left(x^{1}, \ldots, x^{n}\right), x^{i} \in \boldsymbol{R}$ (real numbers); $|x|=\left(\sum\left|x^{i}\right|^{2}\right)^{1 / 2} ;$ if $y=\left(y^{1}, \ldots, y^{n}\right)$, then the inner product $\langle x, y\rangle=\sum x^{i} y^{i} . B(x, r)$ is the open ball of radius $r$ centered at $x$ and $Q(x, r)$ the cube centered at $x$, parallel to the axes of $\boldsymbol{R}^{n}$ with edge $2 r$ :

$$
Q(x, r)=\left\{y \in \boldsymbol{R}^{n} ;\left|y^{i}-x^{i}\right|<r, i=1,2, \ldots, n\right\} .
$$

The diameter, $\operatorname{diam} Q(x, r)$, of the cube is then equal to $2 r \sqrt{n}$. Occasionally it will be convenient to use "half-closed" cubes, defined by the inequalities

$$
x^{i}-r<y^{i} \leqq x^{i}+r, \quad i=1,2, \ldots, n .
$$

For half-closed cubes we shall use the notation $\hat{Q}(x, r)$. Let $\sigma$ be a positive number. Then $\sigma B$ or $\sigma Q$ stands for the ball or a cube, respectively, with the same centre 
as $B$ or $Q$ but contracted (if $\sigma \leqq 1$ ) or expanded (if $\sigma \geqq 1$ ) by the factor of $\sigma$. $\Omega$ will be an open subset of $\boldsymbol{R}^{n}$. $\Omega$ is a domain if it is also connected. The distance function $d(x)$ is defined by $d(x)=\operatorname{dist}(x, \partial \Omega)$, where $\partial \Omega$ is the boundary of $\Omega$. This function is uniformly Lipschitz continuous, i.e.,

$$
|d(x)-d(y)| \leqq|x-y| \text { for all } x, y \in \boldsymbol{R}^{n} .
$$

For a set $F \subset \boldsymbol{R}^{n}$ we write $F \subset \subset \Omega$ if the closure $\bar{F}$ of $F$ is a compact subset of $\Omega$ and we say that $F$ is strictly contained in $\Omega$. This fact is characterized by the inequality

$$
\operatorname{dist}(F, \partial \Omega)=\inf _{x \in F} d(x)>0 \text { for } F \subset \Omega .
$$

We use the notation Int $F$ for the interior set of $F \subset \boldsymbol{R}^{n}$; this is the union of all open balls contained in $F$.

In what follows we shall use the dyadic division of a cube $Q_{0} \subset \boldsymbol{R}^{n}$. We define by induction the families $M_{k}, k=0,1,2, \ldots$ of open subcubes of the cube $Q_{0}$; $M_{0}=\left\{Q_{0}\right\}$. Suppose that the family $M_{k}$ is given. Then we divide dyadically every cube of $M_{k}$ into $2^{n}$ equal cubes. They form together the family $M_{k+1}$, the next generation after $M_{k}$ of the dyadic subdivision. The cubes of $M_{k}$ are disjoint. In general every two cubes from the union $M=\cup_{k} M_{k}$ are either disjoint or one includes the other. The family $\left\{M_{k}\right\}, k=0,1,2, \ldots$ we call the dyadic decomposition of the cube $Q_{0}$. If the dyadic division process is started from a "halfclosed" cube $\hat{Q}_{0}$, we will obtain only half-closed cubes in the process. They have the important property that all cubes of the $k$-th generation $\hat{M}_{k}$ are disjoint in the set theoretic sense and $\hat{Q}_{0}=\bigcup_{\hat{Q} \in \hat{M}_{k}} \hat{Q}$ for each $k$.

It follows that for every $x \in \hat{Q}_{0}$ there exists a unique sequence of half-closed subcubes $\left\{\hat{Q}_{k}(x)\right\}, \hat{Q}_{k+1}(x) \subset \hat{Q}_{k}(x)$ and $\hat{Q}_{k}(x)$ is a cube of the $k$-th generation $\hat{M}_{k}$ containing $x$. These cubes shrink into $x$.

Lemma 1.1. Let $\mathscr{F}$ be a family of open cubes in $\boldsymbol{R}^{n}$ such that the union $\cup_{Q \in \mathscr{F}} Q$ is a bounded set in $\boldsymbol{R}^{n}$. Then there exists an at most countable subfamily $\mathscr{F}^{\prime}$ consisting of disjoint cubes such that

$$
\bigcup_{Q \in \mathscr{F}} Q \subset \bigcup_{Q \in \mathscr{F}^{\prime}} 5 Q .
$$

Moreover, if $\mathscr{F}$ is a subfamily of the dyadic decomposition of a cube $Q_{0}$, then the subfamily $\mathscr{F}^{\prime}$ can be chosen in such a way that

$$
\bigcup_{Q \in \mathscr{F}} Q \subset \bigcup_{Q \in \mathscr{F}^{\prime}} Q .
$$

Proof. Let $\varrho$ be strictly greater than the upper bound for the diameters of the cubes of $\mathscr{F}$. Let $\mathscr{F}, i=0,1,2, \ldots$ be the subfamily of those cubes $Q \in \mathscr{F}$ which satisfy the inequality $2^{-i-1} \varrho<\operatorname{diam} Q \leqq 2^{-i} \varrho$. For $i \neq j$ the subfamilies $\mathscr{F}_{i}$ and $\mathscr{F}_{j}$ are disjoint. Obviously each subfamily of disjoint cubes in $\mathscr{F}_{i}$ is finite. After these remarks we can start the process of constructing the family $\mathscr{F}^{\prime}$. We construct 
$\mathscr{F}^{\prime}$ as $\bigcup_{i} \mathscr{F}_{i}^{\prime}$ with each $\mathscr{F}_{i}^{\prime}$ consisting only of a finite number of cubes. $\mathscr{F}_{0}^{\prime}$ will be a maximal subfamily of disjoint cubes in $\mathscr{F}_{0}$. We exclude now from $\mathscr{F}_{1}$ all cubes which have a non-empty intersection with a cube in $\mathscr{F}_{0}^{\prime}$. From those which remained we consider a maximal subfamily $\mathscr{F}_{1}^{\prime}$ of disjoint cubes. Continuing this process we obviously exhaust all cubes from $\mathscr{F}$. By an elementary geometric observation we have the inclusion

$$
\bigcup_{Q \in \mathscr{F}_{i+1}} Q \subset \bigcup_{Q \in \mathscr{F}_{i}^{\prime} \cup \mathscr{F}_{i+1}^{\prime}} 5 Q, \quad i=0,1,2, \ldots
$$

Hence the family $\mathscr{F}^{\prime}=\bigcup_{i} \mathscr{F}_{i}^{\prime}$ satisfies all requirements of the lemma. In the additional assumption of the lemma we have instead of (1.4) the inclusion

$$
\bigcup_{Q \in \mathscr{F}_{i}^{\prime}} Q \subset \bigcup_{Q \in \mathscr{F}_{i}} Q
$$

This completes the proof of Lemma 1.1.

1.2. Measure and integration. For Lebesgue measure in $\mathbf{R}^{n}$ we use the symbol $d x$. If $E$ is a measurable subset in $\boldsymbol{R}^{n}$, then its measure will be denoted by $|E|$ or mes $E$. For a ball $B$ or a cube $Q$ we have

$$
|\sigma B|=\sigma^{n}|B| \quad \text { or } \quad|\sigma Q|=\sigma^{n}|Q|, \quad \sigma>0 .
$$

If the family $\mathscr{F}$ from Lemma 1.1 is indexed by points of a measurable set $E$ with the only condition that $x \in Q_{x} \in \mathscr{F}$ for each point $x$ of $E$, then Lemma 1.1 reduces to a simple version of Vitali's covering theorem [36].

Lemma 1.2. Let $E$ be a measurable subset of $\boldsymbol{R}^{n}$ which is covered by the union of a family of cubes $\left\{Q_{j}\right\}$, of bounded diameter. Then one can select a subsequence, $Q_{1}, Q_{2}, \ldots$, of disjoint cubes such that

$$
\text { mes } E \leqq 5^{n} \sum_{k}\left|Q_{k}\right| \text {. }
$$

We say that $x \in \boldsymbol{R}^{n}$ is a point of density of $E$ if

$$
\lim _{r \rightarrow 0} \frac{\operatorname{mes} B(x, r) \cap E}{\operatorname{mes} B(x, r)}=1 .
$$

More generally, if $f$ is an integrable function defined on an open set $\Omega \subset \boldsymbol{R}^{\boldsymbol{n}}$, then the point $x \in \Omega$ is said to be the Lebesgue point of $f$ if

$$
\lim _{r \rightarrow 0} \frac{1}{|B(x, r)|} \int_{B(x, r)}|f(y)-f(x)| d y=0 .
$$

The well-known Lebesgue's differentiation theorem says that almost every point of $\Omega$ is the Lebesgue point of $f$.

For $f=\chi_{E}$, the characteristic function of the Lebesgue measurable set $E \subset \Omega$, we get that almost every point $x \in E$ is the point of density of $E$. 
The above definitions remain valid if we replace the balls $B(x, r)$ by cubes $Q(x, r)$. For the average value $(1 /|E|) \int_{E} f(y) d y$ of an integrable function $f$ on a set $E \subset \boldsymbol{R}^{n}$ of positive measure we use the notation

$$
f_{E} f(y) d y \text { or } f_{E} \text {. }
$$

Let $f \in L^{1}(\Omega)$. Then the Lebesgue theorem implies that for almost every $x \in \Omega$

$$
f(x)=\lim _{j \rightarrow \infty} f_{B_{j}} f(y) d y=\lim _{j \rightarrow \infty} f_{Q_{j}} f(y) d y,
$$

where $\left\{B_{j}\right\}\left(\left\{Q_{j}\right\}\right)$ is an arbitrary sequence of balls (cubes) containing $x$ and such that $\lim _{j \rightarrow \infty}\left|B_{j}\right|=0\left(\lim _{j \rightarrow \infty}\left|Q_{j}\right|=0\right)$. A more specialized choice of cubes from the dyadic decomposition $\left\{M_{k}\right\}$ leads to the important Calderon-Zygmund decomposition lemma:

Lemma 1.3. Let $f \in L^{1}\left(Q_{0}\right), f \geqq 0$ and let $t$ be a real number such that $f_{Q_{0}} f(y) d y \leqq t$. Then there exists a countable (or finite) family $\mathscr{F}$ of disjoint subcubes of $Q_{0}$ such that

$$
t<f_{Q} f(y) d y \leqq 2^{n} t \text { for each } Q \in \mathscr{F}
$$

and

$$
f(x) \leqq t \quad \text { for almost every } \quad x \in Q_{0}-\bigcup_{Q \in \mathscr{F}} Q .
$$

Proof. We shall consider the dyadic decomposition $\left\{M_{k}\right\}, k=0,1,2, \ldots$ of the cube $Q_{0}$. For almost every $x \in \Omega_{0}$, i.e., for $x \in \bigcap_{k=0}^{\infty} \cup_{Q \in M_{k}} Q$, we examine the sequence $\left\{Q_{k}(x)\right\}_{k=0,1, \ldots}$ of cubes such that $Q_{k}(x)$ is the cube from the generation $M_{k}$ which contains the point $x$. By the Lebesgue differentiation theorem

$$
\lim _{k \rightarrow \infty} f_{Q_{k}(x)} f(y) d y=f(x)
$$

for almost every point $x \in Q_{0}$. Therefore on a subset $A \subset\left\{x \in Q_{0} ; f(x)>t\right\}$ of full measure (with respect to the set $\left\{x \in Q_{0} ; f(x)>t\right\}$ ) for some sufficiently big natural $k, k \geqq k(x)$

$$
f_{Q_{k}(x)} f(y) d y>t
$$

Since $f_{Q_{0}} f(y) d y \leqq t$, it means that for every point $x \in A$ there exists the first index $k=k^{\prime}(x) \geqq 1$ such that

$$
\underset{Q_{k}(x)}{f} f(y) d y>t \text { and } \underset{Q_{k-1}(x)}{f} f(y) d y \leqq t .
$$


Since $\left|Q_{k-1}(x)\right|=2^{n}\left|Q_{k}(x)\right|$, we have

$$
t<\underset{Q_{k}(x)}{f} f(y) d y \leqq 2^{n} \underset{Q_{k-1}(x)}{f} f(y) d y \leqq 2^{n} t
$$

and get the property (1.10). In this way we obtain a family $\left\{Q_{k}(x)\right\}_{x \in A}$ of cubes indexed by the points of the set $A$. By Lemma 1.1 (see the formula (1.3)) we can select a countable or finite subfamily $\mathscr{F}$ of disjoint cubes covering $A$. If $x \in Q_{0}-$ $\cup_{Q \in \mathscr{F}} Q$, then $x \notin A$; hence for each $k=0,1,2, \ldots f_{Q_{k}(x)} f(y) d y \leqq t$ and the Lebesgue theorem gives (1.11).

1.3. $L^{p}$ spaces. Let $E$ be a measurable subset of $\boldsymbol{R}^{n}, 1 \leqq p<\infty$. We denote by $L^{p}(E)$ the space of functions defined on $E$, such that $|f(x)|^{p}$ is integrable with respect to the measure $d x$ with the norm

$$
\|f\|_{p}=\|f\|_{L^{p}(E)}=\left(\int_{E}|f(x)|^{p} d x\right)^{1 / p},
$$

where the values of $f$ may be complex numbers, vectors or matrices. The Hölder inequality

$$
\|f\|_{p} \leqq\|f\|_{p_{1}}^{\alpha_{1}}\|f\|_{p_{2}}^{\alpha_{2}} \quad \text { for } \quad \frac{1}{p}=\frac{\alpha_{1}}{p_{1}}+\frac{\alpha_{2}}{p_{2}}, \alpha_{1}, \alpha_{2} \geqq 0, \alpha_{1}+\alpha_{2}=1
$$

will be frequently used in its various special cases. For example, it implies that the function $p \rightarrow\left(f_{E}|f|^{p}\right)^{1 / p}$ is increasing, i.e.,

$$
\left(f|f|^{p}\right)^{1 / p} \leqq\left(f_{E}|f|^{q}\right)^{1 / q} \text { whenever } \quad 1 \leqq p \leqq q \leqq \infty .
$$

In the case $q=\infty$ we mean $\left(f_{E}|f|^{q}\right)^{1 / q}=\operatorname{ess~} \sup _{x \in E}|f(x)|$. If $\Phi$ is a positive increasing and convcx function defined on the interval $(-\infty, \infty)$, we have Jensen's inequality

$$
\Phi\left(f_{E} f\right) \leqq f_{E} \Phi(f)
$$

For an open set $\Omega$ the space $L_{\mathrm{loc}}^{p}(\Omega)$ consists of functions which belong to $L^{p}(F)$ for every compact $F \subset \Omega$.

Let $f$ be a measurable function defined on a measurable set $\Omega \subset \boldsymbol{R}^{n}$. We will work with the sets

$$
E_{t}=\{x \in \Omega ;|f(x)|>t\} .
$$

The real-valued function $\lambda(t)=$ mes $E_{t}$ is called the distribution function of $|f|$. Obviously $\lambda(t)$ is a non-increasing function on the interval $[0, \infty])$.

The $L^{p}$ norm of $f$ can be expressed by means of the distribution function $\lambda(t)$ as follows:

$$
\int_{\Omega}|f(x)|^{p} d x=p \int_{0}^{\infty} t^{p-1} \lambda(t) d t
$$


We also have

$$
\int_{|f|(x)>t}|f(x)|^{p} d x=-\int_{t}^{\infty} \tau^{p-1}\left(\int_{|f|>\tau}|f(x)| d x\right)^{\prime} d \tau .
$$

A good source for the material of this and the following subsection is the book of E. Stein [36].

1.4. Sobolev spaces. The basic tool in the study of quasiregular mappings is the theory of functions having weak derivatives. We collect here some results on Sobolev spaces which are necessary for our investigations.

Let $\Omega$ be a domain in $\boldsymbol{R}^{n}$. The symbols $C(\Omega), C_{0}(\Omega), C^{k}(\Omega), C_{0}^{k}(\Omega)$ denote the usual function spaces. If $u$ is a real function of the class $C^{1}(\Omega)$, we denote by $u_{x^{i}}=\partial u / \partial x^{i}$ its partial derivative with respect to $x^{i}$ and by $\nabla u=\left(u_{x^{1}}, \ldots, u_{x^{n}}\right)$ the gradient of $u$. For a mapping $f: \Omega \rightarrow \boldsymbol{R}^{n}$ of the class $C^{1}(\Omega), f=\left(f^{1}, f^{2}, \ldots, f^{n}\right)$ its Jacobi matrix is denoted by

$$
D f(x)=\left(\begin{array}{l}
f_{x^{1}}^{1}, f_{x^{2}}^{1}, \ldots, f_{x^{n}}^{1} \\
f_{x^{1}}^{2}, f_{x^{2}}^{2}, \ldots, f_{x^{n}}^{2} \\
\ldots \ldots \ldots \ldots \ldots \ldots \\
f_{x^{1}}^{n}, f_{x^{2}}^{n}, \ldots, f_{x^{n}}^{n}
\end{array}\right)
$$

and the transposed matrix is $D^{*} f(x)=(D f(x))^{*}$. The Jacobian $J_{f}(x)$ is the determinant of $D f(x)$. Geometrically $D f(x)$ may be interpreted as the linear map between tangent spaces $T_{x} \Omega$ into $T_{f(x)} R^{n}$ which we identify with the Euclidean space $\boldsymbol{R}^{n}$ with the standard inner product $\langle$,$\rangle . The norm of D f(x)$ is then equal to

$$
|D f(x)|=\sup _{|h|=1}|D f(x) h|,
$$

where the supremum is taken over all unit vectors $h$ in $\boldsymbol{R}^{n}$. Sometimes it is convenient to use the norm $\|D f\|^{2}=\operatorname{Tr}\left(D^{*} f D f\right)=\sum_{i, j}\left(f_{x^{i}}^{j}\right)^{2}$.

Suppose we are given a locally integrable function $u$ on $\Omega$. We say that a locally integrable vector function $g$ is the weak gradient of $u$ if for all vector valued test functions $\varphi$ of the class $C_{0}^{1}(\Omega)$ the integral equality

$$
\int_{\Omega} u(x) \operatorname{div} \varphi(x) d x=-\int_{\Omega}\langle g(x), \varphi(x)\rangle d x
$$

holds; here $\operatorname{div} \varphi(x)$ denotes the divergence of $\varphi$, i.e.,

$$
\operatorname{div} \varphi(x)=\sum_{i=1}^{n} \frac{\partial \varphi^{i}}{\partial x^{i}}
$$

The components of $g(x)$ are called the weak or generalized partial derivatives of $u$ and are denoted by the same symbols as in the classical case. 
If $f: \Omega \rightarrow \boldsymbol{R}^{n}$ is a locally integrable mapping, then its generalized Jacobi matrix $D f(x)$ is defined by the conditions $D f \in L_{\mathrm{loc}}^{1}(\Omega)$ and

$$
\int_{\Omega} D^{*} f(x) \varphi(x) d x=-\int_{\Omega} D^{*} \varphi(x) f(x) d x
$$

for every $C_{0}^{\mathbf{1}}(\Omega)$ mapping $\varphi: \Omega \rightarrow \boldsymbol{R}^{n}$.

The symbol $W_{p}^{1}(\Omega)\left(W_{p, \text { loc }}^{1}(\Omega)\right), 1 \leqq p<\infty$ stands for the class of functions or mappings which belong to $L^{p}(\Omega)\left(L_{\mathrm{loc}}^{p}(\Omega)\right)$ and whose weak partial derivatives exist and also belong to $L^{p}(\Omega)\left(L_{\mathrm{loc}}^{p}(\Omega)\right)$. A norm in $W_{p}^{1}(\Omega)$ may be introduced by

$$
\|u\|_{W_{p}^{1}(\Omega)}=\|u\|_{L^{p}(\Omega)}+\|\nabla u\|_{L^{p}(\Omega)} .
$$

This makes $W_{p}^{1}(\Omega)$ into a Banach space. Smooth functions are dense in $W_{p}^{1}(\Omega)$ but the completion of $C_{0}^{\infty}(\Omega)$ in $W_{p}^{1}(\Omega)$ leads to an important closed subspace of $W_{p}^{1}(\Omega)$, which we denote by $\stackrel{\circ}{W}_{p}^{1}(\Omega)$. Returning to the identities (1.20) and (1.22), we remark that using the Hölder inequality one gets

$$
\left\{\begin{array}{l}
\left|\int_{\Omega} u(x) \operatorname{div} \varphi(x) d x\right| \leqq C_{1}\left(\int_{\Omega}|\varphi(x)|^{q} d x\right)^{1 / q} \\
|| \int_{\Omega} D^{*} \varphi(x) f(x) d x \mid \leqq C_{2}\left(\int_{\Omega}|\varphi(x)|^{q} d x\right)^{1 / q},
\end{array}\right.
$$

where $1 / p+1 / q=1$ and $C_{1}=\|\nabla u\|_{p}, C_{2}=\|D f\|_{p}$ whenever $u$ and $f$ belong to $W_{p}^{1}(\Omega)$.

Conversely, if (1.23) holds for every test function $\varphi \in C_{0}^{1}(\Omega)$, then $u$ and $f$ belong to $W_{p}^{1}(\Omega)$ and $\|\nabla u\|_{p} \leqq C_{1},\|D f\|_{p} \leqq C_{2}$. This fact is rather important for practical use; it is a simple consequence of the Hahn-Banach theorem. It is important to note that Lipschitz continuous functions $u \in \operatorname{Lip}\left(\Omega^{\prime}\right)$ are weakly differentiable, that is, $\operatorname{Lip}\left(\Omega^{\prime}\right) \subset W_{\infty}^{1}\left(\Omega^{\prime}\right)$ and the following chain rule holds: if $f: \Omega \rightarrow \Omega^{\prime}$ belongs to the space $W_{p}^{1}(\Omega)$, then the composite function $v(x)=u(f(x))$ also does and $\nabla v(x)=D^{*} f(x) \nabla u(f(x))$ for almost every $x \in \Omega$. For every $f \in W_{p, \text { loc }}^{1}(\Omega), D f(x)=0$ a.e. on any set where $f$ is constant.

In particular, if $u$ and $v$ are real functions of the class $W_{p, \text { loc }}^{1}(\Omega)$, then the function

$$
g(x)=\max \{u(x), v(x)\}=\frac{1}{2}(u(x)+v(x)+|u(x)-v(x)|)
$$

belongs to $W_{p, \text { loc }}^{1}(\Omega)$ and for almost all $x \in \Omega$

$$
\nabla g(x)=\left\{\begin{array}{lll}
\nabla u(x) & \text { if } & u(x) \geqq v(x) \\
\nabla v(x) & \text { if } & u(x) \leqq v(x)
\end{array}\right.
$$

The same holds for $h(x)=\min \{u(x), v(x)\}=(1 / 2)(u(x)+v(x)-|u(x)-v(x)|)$.

Now we pass to the deeper results concerning Sobolev spaces, that is, to the imbedding theorems and $L^{p}$-estimates. Our starting point is the local SobolevPoincare inequality in the standard form. We state without proof the following 
Lemma 1.4. Let $Q$ be a cube or a ball in $\boldsymbol{R}^{n}$. Then for every $u \in W_{p}^{\mathbf{1}}(Q)$, $1 \leqq p<n$ the inequality

$$
\left(\int_{\Omega}\left|u(x)-u_{Q}\right|^{\bar{p}} d x\right)^{1 / \bar{p}} \leqq C(n, p)\left(\int_{\Omega}|\nabla u(x)|^{p} d x\right)^{1 / p}
$$

holds with the universal constant $C(n, p), \bar{p}=n p /(n-p)$. (The best value of $C(n, p)$ in (1.24) of course depends on whether $Q$ is a cube or a ball.)

The proof of this lemma may be easily derived from the classical versions of Sobolev's inequality; see [35].

The local Poincaré's inequality is stated in the following modified form:

Lemma 1.5. If $u \in W_{p}^{1}(Q), 1 \leqq p<\infty$, then for any $0 \leqq \sigma \leqq 1$

$$
\left(f_{Q}\left|u(x)-u_{\sigma Q}\right|^{p} d x\right)^{1 / p} \leqq\left(\frac{2}{\sigma}\right)^{n / p} \operatorname{diam} Q\left(f_{Q}|\nabla u(x)|^{p} d x\right)^{1 / p},
$$

where $Q$ may be a cube or a ball.

Proof. We prove (1.25) under the restriction that $u \in C^{1}(\bar{Q})$. The general case needs only a standard approximation argument. We begin with the formula

$$
u(x)-u(y)=\int_{0}^{1} \frac{d}{d t} u(t x+(1-t) y) d t=\int_{0}^{1}\langle x-y, \nabla u(t x+(1-t) y)\rangle d t
$$

for $x, y \in Q$. Integrating with respect to $y \in \sigma Q$ and applying Hölder's inequality and Fubini's theorem we get

Hence

$$
\begin{gathered}
u(x)-u_{\sigma Q}=\int_{\sigma Q} \int_{0}^{1}\langle x-y, \nabla u(t x+(1-t) y)\rangle d t d y, \\
\left|u(x)-u_{\sigma Q}\right|^{p} \leqq(\operatorname{diam} Q)^{p}\left(f_{\sigma Q} \int_{0}^{1}|\nabla u(t x+(1-t) y)| d t d y\right)^{p} \leqq \\
\leqq(\operatorname{diam} Q)^{p} \int_{\sigma Q} \int_{0}^{1}|\nabla u(t x+(1-t) y)|^{p} d t d y .
\end{gathered}
$$

$$
f_{Q}\left|u(x)-u_{\sigma Q}\right|^{p} d x \leqq(\operatorname{diam} Q)^{p} \int_{0}^{1}\left(f_{Q} f_{\sigma Q}|\nabla u(t x+(1-t) y)|^{p} d x d y\right) d t .
$$

Now for $t$ fixed, change the variables $(x, y) \rightarrow(\xi, \zeta) \in Q \times 2 Q \quad \xi=t x+(1-t) y$, $\zeta=x-y$. Clearly $d x d y=d \xi d \zeta$ and we get

$$
f_{Q}\left|u(x)-u_{\sigma Q}\right|^{p} d x \leqq(\operatorname{diam} Q)^{p}\left(\frac{2}{\sigma}\right)^{n} f_{Q}|\nabla u(\xi)|^{p} d \xi .
$$

This proves (1.25).

The Sobolev and Poincaré inequalities imply the following estimation in the borderline case: 
Lemma 1.6. There exists a constant $v=v(n)>0$ such that for every $v \in W_{n}^{1}(Q)$ and any $0<\sigma \leqq 1$ the inequality

$$
\int_{Q} e^{v \sigma\left|v(x)-v_{\sigma Q}\right| /\|v v\|_{n}} d x \leqq 2
$$

is true; here $Q$ is a cube or a ball and $\|v\|_{n}=\left(\int_{Q}|\nabla v(y)|^{n} d y\right)^{1 / n}$.

Proof. Inequality (1.24) with $p=n / 2$ reads as follows:

Hence

$$
\left(f_{Q}\left|u(x)-u_{Q}\right|^{n} d x\right)^{1 / n} \leqq C(n) \operatorname{diam} Q\left(f_{Q}|\nabla u(x)|^{n / 2} d x\right)^{2 / n} .
$$

$$
\left(f_{Q}|u(x)|^{n} d x\right)^{1 / n} \leqq C(n) \operatorname{diam} Q\left(f_{Q}|\nabla u(x)|^{n / 2} d x\right)^{2 / n}+f_{Q}|u(x)| d x .
$$

Let $\varepsilon>0$. We substitute $e^{\varepsilon\left|v-v_{\sigma Q}\right|}$ for $u$, getting $|\nabla u|=\varepsilon|\nabla v| e^{\varepsilon|v-v|_{\sigma Q}}$ and

$$
\begin{aligned}
& \left(f_{Q} e^{n \varepsilon\left|v-v_{\sigma Q}\right|}\right)^{1 / n} \leqq \varepsilon \cdot C(n) \operatorname{diam} Q\left(f_{Q}|\nabla v|^{n / 2} e^{n \varepsilon\left|v-v_{\sigma Q}\right| / 2}\right)^{2 / n}+f_{Q} e^{\varepsilon\left|v-v_{\sigma Q}\right|} \leqq \\
& \leqq \varepsilon \cdot C(n) \operatorname{diam} Q\left(f_{Q}|\nabla v|^{n}\right)^{1 / n}\left(f_{Q} e^{n \varepsilon\left|v-v_{\sigma Q}\right|}\right)^{1 / n}+1+\varepsilon \int_{Q}\left|v-v_{\sigma Q}\right| e^{\varepsilon\left|v-v_{\sigma Q}\right|} .
\end{aligned}
$$

Here we have used the Hölder inequality and the inequality $e^{t} \leqq 1+t e^{t}$, valid for $t \geqq 0$. Again by the Hölder inequality and by (1.25)

$$
\begin{gathered}
f_{Q}\left|v-v_{\sigma Q}\right| e^{\varepsilon\left|v-v_{\sigma Q}\right|} \leqq\left(f_{Q} e^{n \varepsilon\left|v-v_{\sigma Q}\right|}\right)^{1 / n}\left(f_{Q}\left|v-v_{\sigma Q}\right|^{n}\right)^{1 / n} \\
\leqq \frac{C(n) \operatorname{diam} Q}{\sigma}\left(f_{Q} e^{n \varepsilon\left|v-v_{\sigma Q}\right|}\right)^{1 / n}\left(f_{Q}|\nabla v|^{n}\right)^{1 / n} .
\end{gathered}
$$

Therefore

$$
\left(f_{Q} e^{n \varepsilon\left|v-v_{\sigma Q}\right|}\right)^{1 / n} \leqq 1+\frac{\varepsilon C(n)}{\sigma}\left(f_{Q} e^{n \varepsilon\left|v-v_{\sigma Q}\right|}\right)^{1 / n}\|\nabla v\|_{n} .
$$

Here and later the same letter $C(n)$ is used to denote various constants depending on the dimension only. The lemma immediately follows from the last estimation if we put $\varepsilon=\sigma /\left(2 C(n)\|\nabla v\|_{n}\right)$.

Let now $p$ be strictly greater than $n=\operatorname{dim} \Omega$. Identity (1.26) (with $\sigma=1$ ) implies

$$
\left|u(x)-u_{Q}\right| \leqq(\operatorname{diam} Q) \int_{0}^{1}\left(f_{Q}|\nabla u(t y+(1-t) x)|^{p} d y\right)^{1 / p} d t .
$$

For $t$ fixed by changing the variable $y \rightarrow \xi=t y+(1-t) x \in Q$ we get the estimation

$$
f_{Q}|\nabla u(t y+(1-t) x)|^{p} d y \leqq t^{-n} f_{Q}|\nabla u(\xi)|^{p} d \xi .
$$


Hence we conclude

$$
\left|u(x)-u_{Q}\right| \leqq(\operatorname{diam} Q)\left(\int_{Q}|\nabla u|^{p}\right)^{1 / 2} \int_{0}^{1} t^{-n / p} d t=\frac{p(\sqrt{n})^{n / p}(\operatorname{diam} Q)^{1-n / p}}{p-n}\|\nabla u\|_{p} .
$$

Therefore if $x, y \in Q$, then

$$
|u(x)-u(y)| \leqq\left|u(x)-u_{Q}\right|+\left|u(y)-u_{Q}\right| \leqq \frac{2 p(\bar{r} n)^{n / p}}{p-n}(\operatorname{diam} Q)^{1-n / p}\left(\int_{Q}|\nabla u|^{p}\right)^{1 / p} .
$$

As a simple consequence of (1.28) the following local form of Sobolev's imbedding lemma is obtained:

Lemma 1.7. Let $Q$ be a cube or a ball in $\boldsymbol{R}^{n}$ and let $u \in W_{p}^{1}(Q)$ with $p>n$. Then for a constant $C(n)$ the following estimate holds:

$$
|u(x)-u(y)| \leqq \frac{p C(n)}{p-n}|x-y|^{1-n / p}\left(\int_{Q}|\nabla u|^{p}\right)^{1 / p} .
$$

In other words, $u$ is Hölder continuous on $Q$ and the Hölder exponent $\alpha=1-n / p>0$.

For another approach to Sobolev's inequality see Lemma 7.1 from Section 7.

1.5. Elementary calculus of differential forms. The calculus of differential forms will be useful and, in a sense, even essential in some global geometric problems of the theory of quasiregular mappings. A general $p$-form $\omega$ in a domain $\Omega$ of $\boldsymbol{R}^{n}$, or a differential form of rank $p$, denoted $p=\operatorname{rk~} \omega, 1 \leqq p \leqq n$, will be written as

$$
\omega=\sum_{1 \leqq i_{1}<\ldots<i_{p} \leqq n} \omega_{i_{1} \ldots i_{p}} d x^{i_{1}} \ldots d x^{i_{p}}
$$

with $\omega_{i_{1} \ldots i_{p}}(x)$, called the coefficients of the $p$-form $\omega$, taken from some function space defined in $\Omega$; usually that will be $C^{k}(\Omega), k=1, \ldots$. However, the case of some subspaces of $W_{p}^{m}(\Omega)$ will also appear. We shall then say that the form $\omega$ belongs to the respective function space. The product $d x^{i_{1}} \wedge \ldots \wedge d x^{i_{p}}$ in $(1.31)$ is the exterior product of differentials $d x^{i}$, obeying the anticommutation rules $d x^{i} \wedge d x^{j}=-d x^{j} \wedge d x^{i}$. The general anticommutation relation for the exterior product is $\omega \wedge \varrho=(-1)^{p q} \varrho \wedge \omega$ if $\operatorname{rk} \omega=p$ and $\operatorname{rk} \varrho=q$. For smooth or $C^{k}(\Omega)$ forms the operator of exterior differentiation $d$ is defined by the formula $d \omega=$ $\sum d \omega_{i_{1} \ldots i_{p}} \wedge d x^{i} \wedge \ldots \wedge d x^{i_{p}}$ with $d \omega_{i_{1} \ldots i_{p}}$ being the differential of the function $\omega_{i_{1} \ldots i_{p}}$,

$$
d \omega_{i_{1} \ldots i_{p}}=\sum_{j=1}^{n} \frac{\partial \omega_{i_{1} \ldots i_{p}}}{\partial x^{j}} d x^{j}
$$

The operator $d$ is connected with the exterior multiplication as follows:

$$
d\left(\omega_{1} \wedge \omega_{2}\right)=d \omega_{1} \wedge \omega_{2}+(-1)^{p} \omega_{1} \wedge d \omega_{2},
$$

where $p=\mathrm{rk} \omega_{1}$. Moreover $d^{2}=d \circ d=0$. 
The form $\omega$ such that $d \omega=0$ is called closed while the form $\omega=d \Psi$ for some $(p-1)$-form $\Psi$ is said to be exact. The pull-back $f^{*} \omega$ is defined for any $p$-form $\omega$ defined in the range of $f\left(\omega(y)=\sum \omega(y)_{i_{1} \ldots i_{p}} d y^{i_{1}} \wedge \ldots \wedge d y^{i_{p}}\right)$ as the $p$-form in $\Omega$

$$
f^{*} \omega(x)=\sum \omega(f(x))_{i_{1} \ldots i_{p}} d f^{i_{1}} \wedge \ldots \wedge d f^{i_{p}}, \quad y=f(x), x \in \Omega .
$$

It preserves the exterior product $f^{*}(\omega \wedge \varrho)=f^{*} \omega \wedge f^{*} \varrho$ and commutes with differentiation $d f^{*} \omega=f^{*} d \omega$.

If we speak about the form $\omega$ defined on a closed domain $\bar{\Omega} \subset \boldsymbol{R}^{n}$, we understand that $\omega$ is defined on some open neighbourhood of $\bar{\Omega}$ in $\boldsymbol{R}^{n}$. The restriction $\left.\omega\right|_{\partial \Omega}$ of the form $\omega$ in $\bar{\Omega}$ to the boundary submanifold $\partial \Omega$ of a regular domain $\Omega$ is defined as $\left.\omega\right|_{\partial \Omega}=i^{*} \omega$, where $i: \partial \Omega \rightarrow \bar{\Omega}$ is the natural inclusion.

Integration of a smooth $(n-1)$-form $\omega$ defined on $\Omega$ obeys the fundamental Stokes's formula

$$
\int_{\Omega} d \omega=\int_{\partial} \omega
$$

which we shall use only in most simple cases.

The norm $\|\omega(x)\|$ at the point $x \in \Omega$ of the 1 -form $\omega=\sum_{i=1}^{n} \omega_{i}(x) d x^{i}$ is defined as coming from the Euclidean scalar product $\langle\omega, \varrho\rangle(x)=\sum \omega_{i}(x) \varrho_{i}(x)$, $\|\omega\|^{2}=\langle\omega, \omega\rangle$. The scalar product (at the point $x \in \Omega$ ) of two $p$-forms $\omega=$ $\Theta_{1} \wedge \ldots \wedge \Theta_{p}, \varrho=\tau_{1} \wedge \ldots \wedge \tau_{p}\left(\Theta_{i}\right.$ and $\tau_{i}$ are 1-forms $)$ is defined as

$$
\langle\omega, \varrho\rangle=\operatorname{det}\left\langle\Theta_{i}, \tau_{j}\right\rangle
$$

and extended to general $p$-forms by linearity. Then the elementary Hadamard inequality

$$
\left\|\Theta_{1} \wedge \ldots \wedge \Theta_{n}\right\| \leqq\left\|\Theta_{1}\right\| \ldots\left\|\Theta_{n}\right\|
$$

holds and expresses the fact that the volume of the $n$-parallelepiped spanned by the forms (covectors) $\Theta_{1}, \ldots, \Theta_{n}$ does not exceed the volume of the coordinate $n$-rectangle in $\boldsymbol{R}^{n}$ with edges equal to the length of the edges of the parallelepiped.

1.6. Divergence free vectors and adjugate Jacobian. A vector function $v=$ $\left(v^{1}, \ldots, v^{n}\right), v: \Omega \rightarrow \boldsymbol{R}^{n}$ is said to be divergence free in the domain $\Omega$ if $v \in L_{\text {loc }}^{1}(\Omega)$ and for each test function $\varphi \in C_{0}^{\infty}(\Omega)$

$$
\int_{\Omega}\langle v(x), \nabla \varphi(x)\rangle d x=0
$$

If $v \in W_{1, \text { loc }}^{1}(\Omega)$, then the integration by parts reduces (1.33) to the first order partial differential equation

$$
\operatorname{div} v=\sum_{\alpha} \frac{\partial v^{\alpha}}{\partial x^{\alpha}}=0
$$

An important example of divergence free vectors will be the columns of the so-called adjugate Jacobian. If $A$ is a square $n \times n$ matrix, we define the adjugate 
matrix $\operatorname{adj} A$ by the algebraic identity

$$
A \text { adj } A=\operatorname{det} A \cdot I,
$$

where $I$ is the unit matrix. So, if $A$ is invertible, $\operatorname{adj} A=\operatorname{det} A A^{-1}$. The entries of $\operatorname{adj} A$ are $(n-1)$ homogeneous polynomials with respect to the entries of $A$. The components of $\operatorname{adj} A$ are called cofactors of the components of $A$.

The explicit formula for the entries of $\operatorname{adj} A$ may by written

$$
(\operatorname{adj} A)_{p}^{q}=\sum_{k_{1} \ldots k_{n}} \varepsilon_{k_{1} \ldots k_{n}} A_{1}^{k_{1}} A_{2}^{k_{2}} \ldots A_{p-1}^{k_{p-1}} \delta_{q}^{k_{p}} A_{p+1}^{k_{p+1}} \ldots A_{n}^{k_{n}}
$$

where $\varepsilon_{k_{1} \ldots k_{n}}$ is the Kronecker's fundamental covariant tensor, $\varepsilon_{1,2, \ldots, n}=1$, $\varepsilon_{k_{1}, \ldots, k_{n}}=0$ unless all $k_{i}$ are distinct; $\varepsilon_{k_{1} \ldots, k_{n}}$ changes its sign if two $k_{i}$ are interchanged and $\delta_{q}^{p}$ is defined likewise.

If $f: \Omega \rightarrow \boldsymbol{R}^{n}$ is a mapping, then we denote by $\operatorname{adj} f=\operatorname{adj}(D f)$ the adjugate Jacobian of $f$.

Lemma 1.9. Let $f: \Omega \rightarrow \boldsymbol{R}^{n}$ be a mapping of the class $W_{n-1,10 c}^{1}(\Omega)$. Then each column of the adjugate Jacobian adj $f$ of $f$ is a divergence free vector.

Proof. If $f$ is of the class $C^{2}(\Omega)$, the entries of the adjugate Jacobian adj $f$ are continuously differentiable and the equality $\operatorname{div} \operatorname{adj} f=0$ follows by direct differentiation of columns of $\operatorname{adj} f$. Indeed, by (1.36) we have

$$
\begin{aligned}
& \sum_{p=1}^{n} \frac{\partial(\operatorname{adj} f)_{q}^{p}}{\partial x^{p}}=\sum_{p=1}^{n} \sum_{\substack{k_{1} \ldots k_{n} \\
k_{p} \neq q}} \varepsilon_{k_{1} \ldots k_{n}} \frac{\partial}{\partial x^{p}}\left(\frac{\partial f^{k}}{\partial x^{1}} \ldots \frac{\partial f^{k_{p-1}}}{\partial x^{p-1}} \frac{\partial f^{k_{p+1}}}{\partial x^{p+1}} \ldots \frac{\partial f^{k_{n}}}{\partial x^{n}}\right) \\
& \sum_{s=1}^{n} \sum_{\substack { i=p \\
\begin{subarray}{c}{k_{1} \ldots k_{n} \\
k_{p}=q \\
k_{i}=s{ i = p \\
\begin{subarray} { c } { k _ { 1 } \ldots k _ { n } \\
k _ { p } = q \\
k _ { i } = s } }\end{subarray}} \varepsilon_{k_{1} \ldots k_{n}}\left(\frac{\partial^{2} f^{s}}{\partial x^{i} \partial x^{p}} \prod_{\substack{v \neq i \\
v \neq p}} \frac{\partial f^{k_{v}}}{\partial x^{v}}\right)=\sum_{s=1}^{n} 0=0
\end{aligned}
$$

This is because the expression

$$
\frac{\partial^{2} f^{s}}{\partial x^{i} \partial x^{p}} \prod_{\substack{v \neq i \\ v \neq p}} \frac{\partial f^{k}}{\partial x^{v}}
$$

is symmetric in $i$ and $p$ but the interchange of $i$ and $p$ results in the interchange of $k_{i}$ and $k_{p}$. Since $\varepsilon_{k_{1} \ldots k_{n}}$ is skew symmetric the sum under consideration vanishes. In a weak form we write the above identity

$$
\int_{\Omega} D \eta(x) \operatorname{adj} f(x) d x=0
$$

for every test mapping $\eta: \Omega \rightarrow \boldsymbol{R}^{n}$ of the class $C_{0}^{1}(\Omega)$. To complete the proof of the lemma we observe that for $f \in W_{n-1, \text { loc }}^{1}(\Omega)$ adj $f$ belongs to $L_{\text {loc }}^{1}(\Omega)$ and by an approximation we extend the validity of (1.37) to such mappings. 
Corollary 1.1. Let $f: \Omega \rightarrow \Omega^{\prime}$ be a mapping of the class $W_{n, \mathrm{loc}}^{1}(\Omega)$ and let $\varphi: \Omega^{\prime} \rightarrow \boldsymbol{R}^{n}$ be a $C^{1}\left(\Omega^{\prime}\right)$ mapping with zero divergence. Then the vector $(\operatorname{adj} f) \varphi(f)$ is divergence free. In particular, $\left.(\operatorname{adj} f) f|| f\right|^{n}$ is divergence free for mappings $f: \Omega \rightarrow \boldsymbol{R}^{n}-\{0\}$ of the class $W_{n, \mathrm{loc}}^{1}(\Omega) \cap C(\Omega)$.

Proof. We may assume that $f \in C^{2}(\Omega)$. The formal calculation yields

$$
\begin{gathered}
\operatorname{div}(\operatorname{adj} f) \varphi(f)=\sum_{p, q} \frac{\partial}{\partial x^{p}}\left[(\operatorname{adj} f)_{q}^{p} \varphi^{q}(f)\right]=\sum_{p, q} \frac{\partial(\operatorname{adj} f)_{q}^{p}}{\partial x^{p}} \varphi^{q}(f) \\
+\sum_{p, q, s}(\operatorname{adj} f)_{q}^{p} \frac{\partial \varphi^{q}}{\partial f^{s}} \frac{\partial f^{s}}{\partial x^{p}}=0+\sum_{q, s}(D f \operatorname{adj} f)_{q}^{s} \frac{\partial \varphi^{q}}{\partial f^{s}}=J_{f}(x) \sum_{q, s} \delta_{q}^{s} \frac{\partial \varphi^{q}}{\partial f^{s}} \\
=J_{f}(x) \sum_{q} \frac{\partial \varphi^{q}}{\partial f^{q}}=0 .
\end{gathered}
$$

Here we have used the identity $(D f \operatorname{adj} f)_{q}^{s}=J_{f} \delta_{q}^{s}$. The last statement of the corollary follows from the elementary equality

$$
\operatorname{div} \frac{y}{|y|^{n}} \equiv 0 \quad \text { for } \quad y \in \boldsymbol{R}^{n}-\{0\}
$$

\section{DEFINITION OF QUASIREGULAR MAPS}

Since we shall study only local properties we will restrict our considerations to mappings

$$
f: \Omega \rightarrow \boldsymbol{R}^{n},
$$

or in coordinates

$$
y^{i}=f^{i}\left(x^{1}, x^{2}, \ldots, x^{n}\right) \quad i=1,2, \ldots, n,
$$

where $\Omega$ is an open subset of $\boldsymbol{R}^{n}$.

We accept the following analytical definition of quasiregular mappings.

Definition 2.1. Let $K$ be constant $K \geqq 1$. The mapping (2.1) is $K$-quasiregular in $\Omega$ if

$$
\begin{gathered}
f \in W_{n, \mathrm{loc}}^{1}(\Omega), \\
|D f(x)|^{n} \leqq K J_{f}(x) \quad \text { for almost every } \quad x \in \Omega .
\end{gathered}
$$

Here $D f(x)$ is the derivative or the tangent map of the map $f$, which, as recalled in Section 1, is meaningful at almost every point $x \in \Omega$ if $f$ is in the Sobolev class $W_{n, \mathrm{loc}}^{1}(\Omega)$.

The smallest constant $K$ for which (2.2) is true in $\Omega$ will be called the dilatation of $f$ in $\Omega$. (In accordance with the literature it should be called outer dilatation; however, in this paper we avoid the word "outer" since we shall not introduce any other concept of dilatation.) A map $f$ is quasiregular in $\Omega$ if it is $K$-quasiregular 
in $\Omega$ for some $K \geqq 1: f$ is said to be quasiconformal if it is quasiregular and $1-1$ in $\Omega$. The norm $|D f|$ is understood as in (1.19) as the operator norm of the linear map of the Euclidean space $\boldsymbol{R}^{n}$.

It should be stressed explicitly that our definition of quasiregular mapping does not assume the continuity of $f$. As a matter of fact we shall prove that the continuity of the mapping $f$ is a consequence of (2.2a) and (2.2b). In particular, $f$ will be quasiconformal if and only if it is a homeomorphism onto an open subset of $\boldsymbol{R}^{n}$. For abbreviation we shall write q.r. or q.c. for quasiregular or quasiconformal mapping, respectively.

The map $f$ is a local homeomorphism at a point $x \in \Omega$ if there exists a neighbourhood $\Omega_{x} \subset \Omega$ of $x$ such that the restriction $\left.f\right|_{\Omega_{x}}$ is a homeomorphism of $\Omega_{x}$ onto $f\left(\Omega_{x}\right)$. The branch set $B_{f}$ or more precisely $B_{f}(\Omega)$ of a (continuous) quasiregular map (2.1) is defined as the set of all $x \in \Omega$ such that $f$ is not a local homeomorphism at $x . B_{f}$ is a relatively closed subset of $\Omega$. In general, for a q.r. map the branch set $B_{f}$ is not empty. If $B_{f}=\emptyset$, then the map $f$ is said to be locally quasiconformal.

As said in the introduction, we do not give here any specific examples of q.r. or q.c. mappings. We only mention that this is by no means a restricted class of mappings: it contains any $C^{1}$ mappings with non-zero Jacobian; the generalized solutions of broad classes of uniformly elliptic systems of first order with two independent variables, the uniform limit of $K$-q.r. maps is $K$-quasiregular. For nontrivial examples of quasiregular maps we refer to the existing literature, e.g. [28] or [32], where more comments on the place of q.r. and q.c. mappings in analysis are made. Here we only mention that our general conclusion in this paper will be that, roughly speaking, $K$-q.r. maps form a class of $n$-dimensional mappings which is closed under uniform convergence or almost uniform convergence on compact subsets (or even under much weaker versions of convergence, e.g. $L^{2}$ convergence) but which preserves all general analytical and geometrical properties of locally invertible $C^{1}$-mappings, except at the branch point set $B_{f}$, where they nevertheless preserve some important properties of geometric and analytical regularity in the sense to be explained later.

In connection with Definition (2.1) one more remark is due: when coupled with the classical Hadamard inequality, (2.2b) takes the form of a "double inequality"

$$
J_{f}(x) \leqq|D f|^{n} \leqq K J_{f}(x) .
$$

Thus (2.2b) can be viewed as an "inverse Hadamard inequality". It is this "double inequality" character of $(2.2 b)$ which is responsible for the remarkable functional and analytical properties of the class of mappings satisfying (2.2b). This point of view was stressed in [8]. We shall see in the next section that a local $L^{p}$-version of (2.3) will take the form of "inverse Hölder inequality" and the modified form of "weak inverse Hölder inequality" - and this fact will have important consequences for the theory of q.r. maps. 
They are connected with the fact that the "inverse Hölder inequality" has the remarkable property of self-improving: when a priori assumed to be valid in the $L^{p}$-norm sense for functions in $L_{\mathrm{loc}}^{p}(\Omega)$, it turns out to hold in the $L_{\mathrm{loc}}^{p+\varepsilon}(\Omega)$ sense for some $\varepsilon>0$, implying, in particular, the increased degree of summability. This fact lies at the basis of the most general regularity theory of quasiregular mappings and weak solutions of p.d.e.

\section{BASIC INTEGRAL INEQUALITIES}

We shall write the fundamental differential inequality (2.2b) in the "weak" integral form

$$
\int_{\Omega} \varphi(x)|D f(x)|^{n} d x \leqq K \int_{\Omega} \varphi(x) J_{f}(x) d x
$$

for any test function $\varphi \geqq 0$ of the class $C_{0}^{1}(\Omega)$.

For studying the integral on the right hand side of (3.1) it is useful to remark that the Jacobian $J_{f}(x)$ of a mapping $f: \Omega \rightarrow \boldsymbol{R}^{n}$ naturally appears when we consider the pull-back $f^{*} v$ to $\Omega$ of the volume form $v=d y^{1} \wedge \ldots \wedge d y^{n}$ defined in the range of $f$

$$
f^{*} v=d f^{1} \wedge \ldots \wedge d f^{n}=J_{f}(x) d x^{1} \wedge \ldots \wedge d x^{n} .
$$

Since $f^{*} v=d\left[(-1)^{k-1} f^{k} d f^{1} \wedge \ldots \wedge \widehat{d f}^{k} \wedge \ldots \wedge d f^{n}\right]=d f^{*} \omega_{k}$, where $\omega_{k}$ is the $(n-1)$ form $(-1)^{k-1} y^{k} d y^{1} \wedge \ldots \wedge \widehat{d y}^{k} \wedge \ldots \wedge d y^{n}$ for $k=1, \ldots, n$ (the circumflex over a term in a formula means that it is to be omitted), (3.2) shows that the volume form is exact and we.can evaluate the integral (3.1) on the right hand side by applying Stokes's formula. We exploit this now.

Let $\varphi$ and $f$ be smooth, say of the class $C^{1}(\Omega)$. Then the integrand in (3.1) can be written $\varphi(x) J_{f}(x) d x=d\left(\varphi f^{*} \omega\right)-d \varphi \wedge f^{*} \omega$ for any (n-1)-form $\omega$ such that $d \omega=d y^{1} \wedge \ldots \wedge d y^{n}$. We then get

$$
\int_{\Omega} \varphi(x) J_{f}(x) d x=-\int_{\Omega} d \varphi \wedge f^{*} \omega .
$$

Lemma 3.1. For any $f \in W_{n}^{1}(\Omega)$ and $\varphi \in C_{0}^{1}(\Omega)$ the estimate

$$
\left|\int_{\Omega} \varphi(x) J_{f}(x) d x\right| \leqq \int_{\Omega}|\nabla \varphi(x)||f(x)-c||D f(x)|^{n-1} d x
$$

holds for every constant vector $c \in \boldsymbol{R}^{n}$.

Note that (3.4) also holds for $\varphi \in \stackrel{\circ}{W}_{n}^{1}(\Omega)$ with $\nabla \varphi$ bounded.

Proof. First we establish (3.4) for $f \in C^{1}(\Omega)$. We take for $\omega$ in (3.3) the form

$$
\omega=\frac{1}{n} \sum_{i=1}^{n}(-1)^{i-1}\left(y^{i}-c^{i}\right) d y^{1} \wedge \ldots \wedge \widehat{d y}^{i} \wedge \ldots \wedge d y^{n}
$$


getting

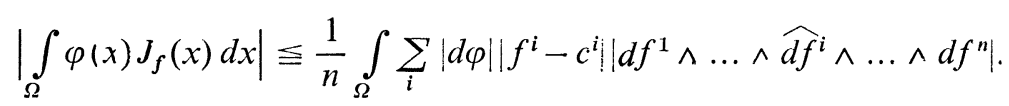

By Hadamard's inequality $\left|d f^{1} \wedge \ldots \wedge \widehat{d f}^{i} \wedge \ldots \wedge d f^{n}\right| \leqq\left|d f^{1}\right| \ldots\left|\widehat{d f}^{i}\right| \ldots\left|d f^{n}\right| \leqq|D f|^{n-1}$ since $\left|d f^{i}\right| \leqq|D f|$ for each $i=1,2, \ldots, n$. Now (3.4) is obtained by the obvious inequality $\left|f^{1}-c^{i}\right| \leqq|f-c|$. If $f \in W_{n}^{1}(\Omega)$ and $\nabla \varphi$ is bounded and $\varphi$ is compactly supported, then $f \in L^{n}(\Omega)$ so $\nabla \varphi|f-c||D f|^{n-1} \in L^{1}(\Omega)$ by Hölder inequality. Therefore by an approximation argument we are led to (3.4) in the general case. From (3.1) and (3.4) we easily get the integral inequality

$$
\left(\int_{\Omega} \Psi^{n}(x)|D f(x)|^{n} d x\right)^{1 / n} \leqq n K\left(\int_{\Omega}|\nabla \Psi(x)|^{n}|f(x)-c|^{n} d x\right)^{1 / n}
$$

valid for $K$-quasiregular mappings $f \in W_{n, \text { loc }}^{1}(\Omega)$, and any non-negative $\Psi$, compactly supported with bounded gradient. Really, put $\varphi=\Psi^{n}$ into (3.1) and (3.4). Then by Hölder inequality

which gives (3.5).

$$
\begin{aligned}
\int \Psi^{n}|D f|^{n} & \leqq K \int|f-c|\left|\nabla \Psi^{n}\right||D f|^{n-1}=n K \int|f-c||\nabla \Psi||\Psi D f|^{n-1} \\
& \leqq n K\left(\int|f-c|^{n}|\nabla \Psi|^{n}\right)^{1 / n}\left(\int \Psi^{n}|D f|^{n}\right)^{(n-1) / n}
\end{aligned}
$$

We apply now (3.5) to the function $\Psi$ with support in a ball $B\left(x_{0}, R\right) \subset \Omega$ such that $\Psi \equiv 1$ in the smaller concentric ball $B\left(x_{0}, \sigma R\right)$, where $0<\sigma<1$. Specifically, we choose $\Psi$ in the form $\Psi(x)=\eta\left(\left|x-x_{0}\right|\right)$ with $\eta(t)=1$ for $0 \leqq t \leqq \sigma R$, $\eta(t)=0$ for $t \geqq R$ and linear for $\sigma R<t<R$. Then

$$
|\nabla \Psi(x)|= \begin{cases}\frac{1}{(1-\sigma) R} & \text { for } \sigma R \leqq\left|x-x_{0}\right| \leqq R \\ 0 & \text { otherwise }\end{cases}
$$

and (3.5) gives for $c=f_{B\left(x_{0}, R\right)}$

$$
\left(f_{B\left(x_{0}, \sigma R\right)}|D f(x)|^{n} d x\right)^{1 / n} \leqq \frac{n K}{\sigma(1-\sigma) R}\left(f_{B\left(x_{0}, R\right)}^{f}\left|f(x)-f_{B\left(x_{0}, R\right)} f\right|^{n} d x\right)^{1 / n} .
$$

From this and the Poincaré inequality (1.24) (with $p=n / 2, \bar{p}=n$ ) we get our basic estimation.

Lemma 3.2. Let $f: \Omega \rightarrow \boldsymbol{R}^{n}$ be a K-quasiregular mapping, and let $B \subset \Omega$ be a ball, $0<\sigma<1$. Then

$$
\left(f_{\sigma B}|D f(x)|^{n} d x\right)^{1 / n} \leqq \frac{K C(n)}{\sigma(1-\sigma)}\left(f_{B}|D f(x)|^{n / 2} d x\right)^{2 / n} .
$$

This estimation remains valid if we replace $B$ by a cube $Q \subset \Omega$. The proof is almost the same.

The last result of this section is the following generalization of Lemma 3.1. 
Lemma 3.3. Assume that the functions $\varphi, \Psi$ and the mappings $f, g$ are of the class $W_{n}^{1}(\Omega)$, that $\nabla \varphi, \nabla \Psi$ are bounded, $\varphi-\Psi \in \dot{W}_{n}^{1}(\Omega)$ and that $f-g \in \dot{W}_{n}^{1}(\Omega)$. Then the following inequality holds:

$$
\begin{gathered}
\left|\int_{\Omega}\left(\varphi J_{f}-\Psi J_{g}\right)\right| \leqq \frac{1}{2} \int_{\Omega}|\nabla(\varphi+\Psi)||f-g|(|D f|+|D g|)^{n-1} \\
+\frac{1}{2} \int_{\Omega}|\nabla(\varphi-\Psi)|\left(|f||D f|^{n-1}+|g||D g|^{n-1}\right)
\end{gathered}
$$

Let us remark that by a proper choice of $\varphi, \Psi$ and $f, g$ we can obtain some useful special cases of (3.8). Thus if $\varphi=\Psi=1$, we have

Proposition 3.1. Let $f, g \in W_{n}^{1}(\Omega)$ and $f-g \in \stackrel{\circ}{W}_{n}^{1}(\Omega)$. Then

$$
\int_{\Omega} J_{f}(x) d x=\int_{\Omega} J_{g}(x) d x
$$

In particular, if $f=\left(f^{1}, f^{2}, \ldots, f^{n}\right)$ is a mapping of the class $W_{n}^{1}(\Omega)$ and one of the components, say $f^{k}, k=1,2, \ldots, n$, vanishes on $\partial \Omega\left(f^{k} \in \stackrel{\circ}{W}_{n}^{1}(\Omega)\right)$, then

$$
\int_{Q} J_{f}(x) d x=0
$$

In fact, for $g=\left(f^{1}, \ldots, f^{k-1}, 0, f^{k+1}, \ldots, f^{n}\right)$ we have $f-g \in \stackrel{\circ}{W}_{n}^{1}(\Omega), \quad J_{g}(x) \equiv 0$ and by (3.9) $\int J_{f}=\int J_{g}=0$.

The proof of Lemma 3.3. As usual, we can assume that $\varphi, \Psi, f$ and $g$ are smooth since the integrals in (3.8) considered as functionals of $\varphi, \Psi, f$ and $g$ are continuous. We write the integral of the left hand side of (3.8) by

$$
\int_{Q}\left(\varphi J_{f}-\Psi J_{g}\right)=\frac{1}{2} \int_{Q}(\varphi+\Psi)\left(J_{f}-J_{g}\right)+\frac{1}{2} \int_{Q}(\varphi-\Psi)\left(J_{f}+J_{g}\right)
$$

and estimate the new integrals separately. By Lemma 3.1 we get

$$
\begin{gathered}
\left|\int_{Q}(\varphi-\Psi)\left(J_{f}+J_{g}\right)\right| \leqq\left|\int_{Q}(\varphi-\Psi) J_{f}\right|+\left|\int_{Q}(\varphi-\Psi) J_{g}\right| \\
\leqq \\
\int_{Q}|\nabla(\varphi-\Psi)|\left(|f||D f|^{n-1}+|g|\left|D_{g}\right|^{n-1}\right) \quad \text { since } \quad \varphi-\Psi \in C_{0}^{1}(\Omega) .
\end{gathered}
$$


Furthermore

$$
\begin{aligned}
& \int_{Q}(\varphi+\Psi)\left(J_{f}-J_{g}\right)=\int_{Q}(\varphi+\Psi)\left(d f^{1} \wedge \ldots \wedge d f^{n}-d g^{1} \wedge \ldots \wedge d g^{n}\right) \\
= & \int_{Q} \sum_{i=1}^{n}(\varphi+\Psi) d f^{1} \wedge \ldots \wedge d f^{i-1} \wedge d\left(f^{i}-g^{i}\right) \wedge d g^{i+1} \wedge \ldots \wedge d g^{n} \\
= & \sum_{i}(-1)^{i-1} \int_{Q} d\left[(\varphi+\Psi)\left(f^{i}-g^{i}\right) d f^{1} \wedge \ldots \wedge d f^{i-1} \wedge d g^{i+1} \wedge \ldots \wedge d g^{n}\right] \\
& -\sum_{i} \int_{Q}\left(f^{i}-g^{i}\right) d f \wedge \ldots \wedge d f^{i-1} \wedge d(\varphi+\Psi) \wedge d g^{i+1} \wedge \ldots \wedge d g^{n} .
\end{aligned}
$$

The first integral appearing in the expression vanishes by Stokes's theorem $\left(f^{i}-g^{i} \in C_{0}^{1}(\Omega)\right)$. Hence

$$
\begin{aligned}
\mid \int_{Q}(\varphi+\Psi) & \left(J_{f}-J_{g}\right)\left|\leqq \int_{Q}\right| f-\left.g|| \nabla(\varphi+\Psi)\left|\sum_{i=1}^{n}\right| D f\right|^{i-1} \mid D g^{\mid n-i} \\
& \leqq \int_{Q}|f-g||\nabla(\varphi+\Psi)|(|D f|+|D g|)^{n-1} .
\end{aligned}
$$

Finally (3.11), (3.12) and (3.13) imply (3.8).

\section{MAXIMAL FUNCTIONS AND INVERSE HÖLDER INEQUALITIES}

4.1. Maximal functions. We shall make use of some techniques connected with the Hardy-Littlewood maximal function operator. However, for our purposes, it will be essential to introduce some generalizations and modifications of the theory.

We shall work in a fixed open cube $Q_{0}$ in $\boldsymbol{R}^{n}$. Therefore the notion of the maximal function will be localized to the cube $Q_{0}$. For a function $f \in L^{p}\left(Q_{0}\right), 1 \leqq p$, we shall define the maximal function of order $p$ by the formula

$$
M_{p} f(x)=\sup \left\{\left(f_{Q}|f|^{p}\right)^{1 / p}: x \in Q \subset Q_{0}\right\},
$$

where the supremum is taken over all parallel open subcubes $Q$ of $Q_{0}$, containing the point $x$. For $M_{1} f(x)$ we shall write $M f(x)$.

Besides the maximal function (3.1) it is also of interest to study the weak maximal function

$$
W M_{p} f(x)=\sup \left\{\left(\int_{\sigma Q}|f|^{p}\right)^{1 / p}: x \in \sigma Q, Q \subset Q_{0}\right\}, \quad 0<\sigma \leqq 1,
$$

and the Morrey type maximal functions

$$
M_{\alpha, p} f(x)=\sup \left\{\left(\frac{1}{|Q|^{\alpha / n}} \int_{Q}|f|^{p}\right)^{1 / p} ; x \in Q \subset Q_{0}\right\} .
$$


The dependence of $W M_{p} f$ on $\sigma$ is not included in the notation, since for the purposes of this paper it is enough to take $\sigma=1 / 2$, which we do henceforth.

For $\alpha=n$ we have $M_{n, p} \equiv M_{p} f$ and for $\alpha=0 \quad M_{0, p} f(x) \equiv\left(\int_{Q_{0}}|f|^{p}\right)^{1 / p}$.

For each $\lambda>0$

$$
M_{\alpha, p} \lambda f=\lambda M_{\alpha, p} f ; \quad M_{\alpha, p}(f+g) \leqq M_{\alpha, p} f+M_{\alpha, p} g .
$$

However, only for $\alpha=n M_{n, p} 1=1$.

The maximal functions $M_{\alpha, p} f$ are closely related with the cubical $\alpha$-dimensional outer Hausdorff measure $\gamma_{\alpha}(F)$ defined for any subset $F \subset \boldsymbol{R}^{n}$ by

$$
\gamma_{\alpha}(F)=\inf _{\mathscr{F}} \sum_{Q \in \mathscr{F}}|Q|^{\alpha / n}
$$

where the infimum is taken over all countable families $\mathscr{F}$ of coordinate parallel subcubes of $\boldsymbol{R}^{n}$ which cover $F\left(F \subset \cup_{Q \in \mathscr{F}} Q\right)$. If $F$ is Lebesgue measurable, then $\gamma_{n}(F)=|F|$. The following lemma is a direct consequence of the Vitali covering lemma:

Lemma 4.1. For every $f \in L^{p}\left(Q_{0}\right)$ and every $t>0$ the inequality

$$
\gamma_{\alpha}\left\{x \in Q_{0} ; M_{\alpha, p} f(x)>t\right\}<\frac{5^{\alpha}}{t^{p}} \int_{Q_{0}}|f(x)|^{p} d x, \quad p \geqq 1
$$

holds. For the case $\alpha=n$ we have the following generalization of (4.4): for $0<\Theta<1$

$$
\operatorname{mes}\left\{x \in Q_{0}, M_{p} f(x)>t\right\}<\frac{5^{n}}{(1-\Theta)^{p} t^{p}} \int_{|f(x)| \geqq \Theta t}|f(x)|^{p} d x \text {. }
$$

Proof. For $t>0$ we study the distribution set $E_{t} f \equiv\left\{x \in Q_{0} ; M_{\alpha, p} f(x)>t\right\}$. Then for each $x \in E_{t} f$ one can find a cube $Q_{x}$ such that $x \in Q_{x} \subset Q_{0}$ and

$$
\int_{Q_{x}}|f(y)|^{p} d y>t^{p}\left|Q_{x}\right|^{\alpha / n}
$$

By Lemma 1.1 we may choose a sequence $Q_{x_{v}}, v=1,2, \ldots$ of disjoint cubes such that $\bigcup_{v} 5 Q_{x_{v}}$ covers $\bigcup_{x \in E_{t} f} Q_{x} \supset E_{t} f$.

Therefore

$$
\begin{gathered}
\gamma_{\alpha}\left(E_{t} f\right) \leqq \sum_{v}\left|5 Q_{x_{v}}\right|^{\alpha / n} \leqq 5^{\alpha} \sum_{v}\left|Q_{x_{v}}\right|^{\alpha / n}<\frac{5^{\alpha}}{t^{p}} \sum_{v} \int_{Q_{x_{v}}}|f|^{p} \leqq \frac{5^{\alpha}}{t^{p}} \int_{\cup Q_{x_{v}}}|f|^{p} \\
\leqq \frac{5^{\alpha}}{t^{p}} \int_{Q_{0}}|f|^{p},
\end{gathered}
$$

which is identical with (4.4).

For $0 \leqq \Theta<1$ we define $f_{\Theta}(x)=f(x)$ if $|f(x)| \geqq t \Theta$ and $f_{\Theta}(x)=0$ if $|f(x)|<t \Theta$. Then $|f(x)| \leqq\left|f_{\Theta}(x)\right|+t \Theta$ for each $x \in Q_{0}$ and we get $M_{p} f(x) \leqq M_{p} f_{\Theta}(x)+t \Theta$, which implies $E_{t} f \subset E_{(1-\Theta) t} f_{\Theta}$. Here we used the additional assumption $\alpha=n$. 
The inequality (4.4) applied to $f_{\theta}$ gives

$$
\left|E_{(1-\Theta) t} f_{\Theta}\right|<\frac{5^{n}}{(1-\Theta)^{p} t^{p}} \int_{Q_{0}}\left|f_{\Theta}\right|^{p}=\frac{5^{n}}{(1-\Theta)^{p} t^{p}} \int_{|f| \geqq t \Theta}|f|^{p},
$$

which immediately leads to (4.5).

The Calderon-Zygmund decomposition lemma allows us to prove some kind of "weak inverse inequality" to (4.5).

Lemma 4.2. Let $t \geqq\left(f_{Q_{0}}|f|^{p}\right)^{1 / p}$. Then for $p \geqq 1$

$$
\left|\left\{x \in Q_{0} ; M_{p} f(x)>t\right\}\right| \geqq \frac{1}{2^{n} t^{p}} \int_{|f|>t}|f|^{p} .
$$

Proof. Define $E_{t} f$ as in the previous proof for $\alpha=n$. Let $\mathscr{F}$ be the family of disjoint subcubes of $Q_{0}$ defined by the decomposition Lemma 1.3. Then

and

$$
|f(x)| \leqq t \text { for almost every } x \in Q_{0}-\bigcup_{Q \in \mathscr{F}} Q
$$

$$
t^{p}<f_{Q} f^{p} \leqq 2^{n} t^{p} \text { for each } Q \in \mathscr{F} \text {. }
$$

In other words, we have the inclusions

$$
\left\{x \in Q_{0} ;|f(x)|>t\right\} \subset \bigcup_{Q \in \mathscr{F}} Q \subset E_{t} f,
$$

the first inclusion being understood as valid up to a set of Lebesgue measure zero. Hence.

$$
\left|E_{t} f\right| \geqq \sum_{Q \in \mathscr{F}}|Q| \geqq \sum_{Q \in \mathscr{F}} \frac{1}{2^{n} t^{p}} \int_{Q}|f|^{p}=\frac{1}{2^{n} t^{p}} \int_{\mathcal{Q} \in \mathscr{F}}|f|^{p} \geqq \frac{1}{2^{n} t^{p}} \int_{|f|>t}|f|^{p} .
$$

This completes the proof of the lemma.

It will be convenient to introduce the distribution function $\lambda_{p}(t)$ of the maximal function $M_{p} f$. For $p=1$ we put $\lambda_{1}(t) \equiv \lambda(t)$. Lemmas 4.1 and 4.2 give some estimates for the distribution function $\lambda_{p}(t)$ for $t \rightarrow \infty$. In particular, they imply that the maximal function $M_{p} f$ is finite almost everywhere in $Q_{0}$ if $f \in L^{p}\left(Q_{0}\right)$ and can be used to prove that the Hardy-Littlewood maximal function operator $M f$ is a bounded operator in $L^{p}\left(Q_{0}\right)$ for $1<p \leqq \infty$.

Lemma 4.3. For any $f \in L^{p}\left(Q_{0}\right), p>1$ the inequality

holds.

$$
f_{Q_{0}}|M f|^{p} \leqq \frac{5^{n} e p^{2}}{p-1} f_{Q_{0}}|f|^{p}
$$


Proof. We use (4.5) for $p=1$ and $0 \leqq \Theta<1$ and also the formula (1.16) and Fubini's theorem, getting

$$
\begin{aligned}
& \int_{Q_{0}}|M f|^{p}=p \int_{0}^{\infty} t^{p-1} \lambda(t) d t \leqq \frac{5^{n} p}{1-\Theta} \int_{0}^{\infty} t^{p-2}\left(\int_{|f|>\Theta t}|f(x)| d x\right) d t \\
= & \frac{5^{n} p}{1-\Theta} \int_{Q_{0}}\left(|f(x)| \int_{0}^{\left|\Theta^{-1} f(x)\right|} t^{p-2} d t\right) d x=\frac{5^{n} p}{(p-1) \Theta^{p-1}(1-\Theta)} \int_{Q_{0}}|f(x)|^{p} d x .
\end{aligned}
$$

Parameter $\Theta=(p-1) / p<1$ minimizes the last expression giving (4.7) with $e \geqq$ $(1+1 /(p-1))^{p-1}$.

The inverse inequality (4.6) implies the important estimate of $L^{p}$-norm of $f$ in terms of the $L^{s}$-norm of $f, s \leqq p$ and $L^{p}$-norm of the maximal function $M_{s} F$.

Lemma 4.4. Let $p \geqq s \geqq 1$ and $f \in L^{p}\left(Q_{0}\right)$. Then

$$
f_{Q_{0}}|f|^{p} \leqq\left(f_{Q_{0}}|f|^{s}\right)^{p / s}+\frac{2^{n}(p-s)}{p} f_{Q_{0}}\left|M_{s} f\right|^{p} .
$$

Proof. Since (4.8) is invariant under the substitution $p^{\prime}=p \tau, s^{\prime}=s \tau, f^{\prime}=|f|^{1 / \tau}$ with some $\tau \geqq 1$, it is easily seen that the general case of (4.8) follows from the case when $s=1$. Therefore we prove it for $s=1$ only. Put $t_{0}=f_{Q_{0}}|f|$. We estimate

$$
\int_{Q_{0}}|f|^{p}=\int_{|f| \leqq t_{0}}|f|^{p}+\int_{|f|>t_{0}}|f|^{p} \leqq t_{0}^{p-1} \int_{|f| \leqq t_{0}}|f|+\int_{|f|>t_{0}}|f|^{p} .
$$

Now in the second integral of the right hand side of (4.9) we apply the formula of integration by parts

$$
\begin{gathered}
\int_{|f|>t_{0}}\left|f^{p}\right|^{p}=-\int_{t_{0}}^{\infty} \tau^{p-1}\left(\int_{|f|>\tau}|f|\right)^{\prime} d \tau=t_{0}^{p-1} \int_{|f|>t_{0}}|f| \\
+(p-1) \int_{t_{0}}^{\infty} \tau^{p-2}\left(\int_{|f|>\tau}|f(x)| d x\right) d \tau .
\end{gathered}
$$

The inequality (4.6) yields

$$
\int_{t_{0}}^{\infty} \tau^{p-2}\left(\int_{|f|>\tau}|f(x)| d x\right) d \tau \leqq 2^{n} \int_{t_{0}}^{\infty} \tau^{p-1} \lambda(\tau) d \tau=\frac{2^{n}}{p} \int_{M f>t_{0}}|M f|^{p} .
$$

Here we have used the formulas (1.16), (1.17). In view of (4.9) we conclude

$$
\int_{Q_{0}}|f(x)|^{p} d x \leqq t_{0}^{p-1} \int_{Q_{0}}|f(x)| d x+\frac{2^{n}(p-1)}{p} \int_{Q_{0}}|M f(x)|^{p} d x,
$$

which is identical with (4.8) for $s=1$.

Combining Lemma 4.3 and (4.4) we get 
Proposition 4.1. Let $s, p$ be such that $1 \leqq s \leqq p, p>1$ and let $f \in L^{p}\left(Q_{0}\right)$. Then

$$
\underset{Q_{0}}{f}|M f|^{p} \leqq \frac{5^{n} e p^{2}}{p-1}\left(f_{Q_{0}}|f|^{s}\right)^{p / s}+\frac{10^{n} e p(p-s)}{p-1} f_{Q_{0}}\left|M_{s} f\right|^{p} .
$$

4.2. Inverse Hölder inequality. The inequality (4.10) is a crucial tool in the following proof of the advertised self-improving character of inverse Hölder inequalities (see F. Gehring [14]).

Theorem 4.1. Assume that the function $f \in L^{s}\left(Q_{0}\right), s>1$, satisfies the inverse Hölder inequalities

$$
\left(f_{Q}|f(x)|^{s} d x\right)^{1 / s} \leqq C_{s} f_{Q}|f(x)| d x
$$

for each subcube $Q \subset Q_{0}$, with the constant $C_{s}$ independent of the cube $Q$. Then for each $p>s$ such that

$$
v=\frac{10^{n} e p(p-s) C_{s}^{p}}{p-1}<1
$$

the function $f$ belongs to $L^{p}\left(Q_{0}\right)$. Moreover

$$
\left(\int_{Q_{0}}|f(x)|^{p} d x\right)^{1 / p} \leqq C_{p} f_{Q_{0}}|f(x)| d x
$$

with the constant $C_{p}$ depending only on $n, p, v$. One can take

$$
C_{p}=\left[\frac{5^{n} e p^{2}}{(p-1)(1-v)}\right]^{1 / p} C_{s}
$$

Proof. The formula (4.11) implies that $M_{s} f(x) \leqq C_{s} M f(x)$ for every $x \in Q_{0}$. Then (4.10) and (4.11) give

$$
(1-v) \int_{Q_{0}}|M f(x)|^{p} d x \leqq \frac{5^{n} e p^{2}}{p-1}\left(f_{Q_{0}}|f(x)|^{s} d x\right)^{p / s} \leqq \frac{5^{n} e p^{2} C_{s}^{p}}{p-1}\left(f_{Q_{0}}|f|\right)^{p} .
$$

Since $|f(x)| \leqq M f(x)$ a.e., the estimate (4.12) follows.

Remark. The inequality (4.12) remains true for every subcube $Q \subset Q_{0}$ with the same exponent $p$ and the same universal constant $C_{p}$. This is clear because we could use the cube $Q$ as our basic cube $Q_{0}$. Theorem 4.1 has been proved for a function $f \in L^{p}\left(Q_{0}\right)$. Therefore, strictly speaking, our proof gives only the inequality (4.12) with the universal constant $C_{p}$ if we a priori know that $f \in L^{p}\left(Q_{0}\right)$. The following observation helps to settle this point. If $f \in L^{s}\left(Q_{0}\right)$ satisfies (4.11), then there exists a sequence of continuous functions $f_{k} \in C\left(\boldsymbol{R}^{n}\right)$ such that $f_{k} \rightarrow f$ in $L^{s}\left(Q_{0}\right)$ and $f_{k}$ satisfies the inverse Hölder inequality (4.11) with the same constant $C_{s}$ for each cube $Q$ strictly contained in $Q_{0}$. Therefore we are justified to apply 
(4.12) to the functions $f_{k}$ getting

$$
\left(f_{Q}\left|f_{k}\right|^{p}\right)^{1 / p} \leqq C_{p} f_{Q}\left|f_{k}\right| \text { for every } Q \subset \subset Q_{0} .
$$

Now the application of the Lebesgue convergence theorem and Fatou's lemma gives the desired conclusion that $f \in L^{p}\left(Q_{0}\right)$.

The explicit proof of these facts is postponed to the proof of the more general Theorem 4.2, where an analogous approximation problem arises.

4.3. Weak inverse Hölder inequality. It is essential for the foundation of the theory of quasiregular mappings in $\boldsymbol{R}^{n}$, as well as for a number of important problems of partial differential equations, to have an analogue of Theorem 4.1 for weak inverse Hölder inequalities. By this we mean inequalities of the form

$$
\left(f_{\sigma Q}|f(x)|^{s} d x\right)^{1 / s} \leqq C_{s} f_{Q}|f(x)| d x
$$

assumed to hold for any coordinate parallel subcube $Q \subset Q_{0}$. Here $\sigma$ is a fixed constant $0<\sigma<1$ and $C_{s}$ is assumed to be independent of the cube $Q$, though in general it will depend on $\sigma$. In this case we have $W M_{s} f(x) \leqq C_{s} M f(x)$. For our purposes it is enough to consider $\sigma=1 / 2$. To handle this more general case we shall need to modify Proposition 4.1 by introducing the localizing weight factor $\varrho(x)$ and the weak maximal function $W M_{p} f(x)$ with $\sigma=1 / 2$ (see Section 4.2). For simplicity we assume that $Q_{0}$ is the unit cube, $Q_{0}=\left\{x \in \boldsymbol{R}^{n} ;\|x\|<1\right\}$ where $\|x\|=\max \left|x^{i}\right|$. As a weight factor $\varrho(x)$ we take the function

$$
\varrho(x)=(1-\|x\|)^{n / s}<1 .
$$

Obviously $\varrho(x)=0$ on the boundary of $Q_{0}$.

Proposition 4.2. Let $s, p$ be such that $1 \leqq s<p, p>1$ and let $f \in L^{p}\left(Q_{0}\right)$. Then

$$
f_{Q_{0}} \varrho^{p}|M f|^{p} \leqq \frac{10^{2 p n}}{p-1}\left(f_{Q_{0}}|f|^{s}\right)^{p / s}+10^{2 p n} \frac{p-s}{p-1} \int_{Q_{0}} \varrho^{p}\left|W M_{s} f\right|^{p} .
$$

Proof. To prove (4.15) we first show two pointwise estimates:

$$
\begin{gathered}
|\varrho(x) M f(x)|^{p} \leqq 2^{n p / s}|M \varrho f(x)|^{p}+3^{n p / s}\left(f_{Q_{0}}|f|^{s}\right)^{p / s} \\
\left|M_{s} \varrho f(x)\right|^{p} \leqq\left(\frac{3}{2}\right)^{n p / s}\left|\varrho(x) W M_{s} f(x)\right|^{p}+3^{n p / s}\left(f_{Q_{0}}|f|^{s}\right)^{p / s} .
\end{gathered}
$$

We derive them simultanously. For a point $x \in Q_{0}$ let $Q$ be an arbitrary coordinate parallel subcube of $Q_{0}$ containing $x$. The two terms on the right hand side of (4.16) and (4.17) correspond to two possible cases. 
Case 1. $1-\|x\| \leqq 2|Q|^{1 / n}$. In this case

and we have

$$
\sup _{y \in Q}(1-\|y\|) \leqq 3|Q|^{1 / n}
$$

Also

$$
\left(f_{Q} \varrho^{s}|f|^{s}\right)^{1 / s} \leqq 3^{n / s}|Q|^{1 / s}\left(f_{Q}|f|^{s}\right)^{1 / s} \leqq 3^{n / s}\left(f_{Q_{0}}|f|^{s}\right)^{1 / s} .
$$

$$
\varrho(x) \underset{Q}{f}|f| \leqq 3^{n / s}|Q|^{1 / s}\left(f_{Q}|f|^{s}\right)^{1 / s} \leqq 3^{n / s}\left(f_{Q_{0}}|f|^{s}\right)^{1 / s} .
$$

Case 2. $1-\|x\|>2|Q|^{1 / n}$. Hence $1-\|x\|>2\|x-y\|$ for every $y \in Q$ and consequently we get

$$
\frac{1}{2}(1-\|x\|)<\inf _{y \in Q}(1-\|y\|) \leqq \sup _{y \in Q}(1-\|y\|)<\frac{3}{2}(1-\|x\|) .
$$

Furthermore, the double cube $2 Q$ is a subcube of $Q_{0}$. In particular,

$$
\frac{2}{3} \leqq \frac{1-\|x\|}{1-\|y\|} \leqq 2 \text { for each } y \in Q .
$$

Therefore we can obviously write

Also

$$
\left(f_{Q}|\varrho f|^{s}\right)^{1 / s}=\varrho(x)\left(f_{Q}\left(\frac{1-\|y\|}{1-\|x\|}\right)^{n}|f(y)|^{s} d y\right)^{1 / s} \leqq\left(\frac{3}{2}\right)^{n / s} \varrho(x)\left(f_{Q}|f|^{s}\right)^{1 / s}
$$

$$
\leqq\left(\frac{3}{2}\right)^{n / s} \varrho(x) W M_{s} f(x) .
$$

The last inequality follows from the fact that $2 Q \subset Q_{0}$. Now (4.19) and (4.20) imply

$$
(\varrho(x) \underset{Q}{f} \mid f)^{p} \leqq 2^{n p / s}|M \varrho f(x)|^{p}+3^{n p^{\prime} s}\left(f_{Q_{0}}|f|^{s}\right\}^{p / s}
$$

and (4.18) together with (4.21) yield

$$
\left(f_{Q}|\varrho f|^{s}\right)^{p / s} \leqq\left(\frac{3}{2}\right)^{p n / s}\left|\varrho(x) W M_{s} f(x)\right|^{p}+3^{n p / s}\left(f_{Q_{0}}|f|^{s}\right)^{p / s} .
$$

These estimates hold for every $x \in Q_{0}$ and each cube $Q$ such that $x \in Q \subset Q_{0}$. By the definition of the maximal functions the estimates (4.16) and (4.17) follow. 
If we integrate (4.16) over $Q_{0}$, then apply (4.10) to the function $\varrho f$ and use (4.17), we get (4.15). In fact

$$
\begin{gathered}
\underset{Q_{0}}{f} \varrho^{p}|M f|^{p} \leqq 2^{n p / s} f_{Q_{0}}|M \varrho f|^{p}+3^{n p / s}\left(f_{Q_{0}}|f|^{s}\right)^{p / s} \leqq \frac{2^{n p / s} 5^{n} e p^{2}}{p-1}\left(f_{Q_{0}}|\varrho f|^{s}\right)^{p / s} \\
+\frac{2^{n p / s} 10^{n} e p(p-s)}{p-1} \int_{Q_{0}}\left|M_{s} \varrho f\right|^{p}+3^{n p / s}\left(f_{Q_{0}}|f|^{s}\right)^{f / s} \\
\leqq
\end{gathered}
$$

Finally simplifying the terms in front of the above integrals we get (4.15). From this Proposition we deduce the fundamental

Theorem 4.2. Assume that the function $f \in L^{s}\left(Q_{0}\right), 1<s$, satisfies the weak inverse Hölder inequality

$$
\left(f_{\sigma Q}|f(x)|^{s} d x\right)^{1 / s} \leqq C_{s} f_{Q_{0}}|f(x)| d x, \text { where } \quad \sigma=\frac{1}{2},
$$

for each subcube $Q$ of $Q_{0}$ with the constant $C_{s}$ independent of the cube $Q$. Then for each $p>s$ such that

$$
v=10^{2 p n} \frac{p-s}{p-1} C_{s}^{p}<1
$$

the function $f$ belongs to $L_{\mathrm{loc}}^{p}\left(Q_{0}\right)$ and the uniform estimates

$$
\left(f_{\sigma Q}|f(x)|^{p} d x\right)^{1 / p} \leqq C_{p}\left(f_{Q}|f(x)|^{s} d x\right)^{1 / s}, \quad \sigma=\frac{1}{2}
$$

hold for each subcube $Q$ strictly contained in $Q_{0}$ with the constant $C_{p}$ depending only on $n, p, v$.

Proof. First we assume that $f$ is continuous in $Q_{0}$. The inequality (4.22) means that $W M_{s} f(x) \leqq C_{s} M f(x)$ for each $x \in Q_{0}$. Then as in the proof of Theorem 4.1, using (4.15), we get

$$
f_{Q_{0}} \varrho^{p}|M f|^{p} \leqq \frac{10^{2 p n}}{p-1}\left(\left.f_{Q_{0}}|f|^{s}\right|^{p / s}+10^{2 p n} \frac{(p-s)}{p-1} C_{s}^{p} f_{Q_{0}} \varrho^{p}|M f|^{p} .\right.
$$

On account of the assumption (4.23)

$$
f_{Q_{0}} \varrho^{p}|M f|^{p} \leqq\left.\frac{10^{2 p n}}{(p-1)(1-v)} \underset{Q_{0}}{f}|f|^{s}\right|^{p / s} .
$$


For $x \in \sigma Q_{0}, \sigma=1 / 2$ we obviously have $\varrho(x) \geqq(1-1 / 2)^{n / s}=2^{-n / s}$ and therefore

$$
\underset{\sigma Q_{0}}{f}|f(x)|^{p} d x \leqq 2^{n} 2^{n p / s} \underset{Q_{0}}{f} \varrho^{p}|M f|^{p} \leqq \frac{10^{3 p n}}{(p-1)(1-v)}\left(f_{Q_{0}}|f|^{s}\right)^{p / s},
$$

which is equivalent to (4.24) with

$$
C_{p}=\frac{10^{3 n}}{[(p-1)(1-v)]^{1 / p}}
$$

and $Q=Q_{0}$. Notice that the exponent $p$ and the constant $C_{p}$ do not depend on the cube $Q_{0}$. Therefore we can apply the same reasoning to any subcube $Q \subset Q_{0}$ getting (4.24).

Finally we eliminate the assumption of the continuity of $f$ by the following approximation argument. We take an arbitrary subcube $Q^{\prime}$ strictly contained in $Q_{0}$ and a mollifier function $\eta(x)$ supported in a sufficiently small neighbourhood of $0 \in \boldsymbol{R}^{n}$ which approximates the Dirac measure concentrated at the origin. The convolution

$$
F(x)=\eta *|f|=\int_{R^{n}} \eta(y)|f(x-y)| d y
$$

is well defined in a neighbourhood of $Q^{\prime}$ and it is continuous. It is easy to verify that for every cube $Q \subset Q^{\prime}$

$$
\begin{gathered}
\left(f_{\sigma Q}|F(x)|^{s} d x\right)^{1 / s}=\left(\int_{\sigma Q} f\left(\int_{R^{n}} \eta(y)|f(x-y)| d y\right)^{s} d x\right)^{1 / s} \\
\leqq \int_{R^{n}} \eta(y)\left(\int_{\sigma Q}|f(x-y)|^{s} d x\right)^{1 / s} d y .
\end{gathered}
$$

Here we used Fubini's theorem and Minkovski's inequality. From (4.22) we get $\left(f_{\sigma Q}|f(x-y)|^{s} d x\right)^{1 / s} \leqq C_{s} f_{Q}|f(x-y)| d x$. Hence

$$
\left(f_{\sigma Q}|F(x)|^{s} d x\right)^{1 / s} \leqq C_{s} \int_{R^{n}} \eta(y) f_{Q}|f(x-y)| d x d y=C_{s} f_{Q}|F(y)| d y .
$$

Now we are justified to write (4.24) for the function $F$

$$
\left(f_{\sigma Q}|F(x)|^{p} d x\right)^{1 / p} \leqq C_{p}\left(f_{Q}|F(x)|^{s} d x\right)^{1 / s}
$$

with $C_{p}$ independent of the mollifier function $\eta$. By Young's inequality

$$
\left(f_{Q}|F|^{s}\right)^{1 / s}=\left[f_{Q}(\eta *|f|)^{s}\right]^{1 / s} \leqq\|\eta\|_{L^{1}\left(R^{n}\right)}\left(f_{Q}|f|^{s}\right)^{1 / s}=\left(f_{Q}|f|^{s}\right)^{1 / s}
$$


and we obtain from (4.25) the uniform estimates

$$
\left(f_{\sigma Q}|F|^{p}\right)^{1 / p} \leqq C_{p}\left(f_{Q}|f|^{s}\right)^{1 / s}
$$

with $C_{p}$ independent of $\eta$. This shows that $|f|=\lim _{j \rightarrow \infty} \eta_{j} *|f|$ belongs to $L^{p}(\sigma Q)$ and satisfies (4.24). The limit is taken over a sequence of mollifier functions $\eta_{j}$ tending to the Dirac measure. This completes the proof of the Theorem.

\section{CONTINUITY AND DIFFERENTIABILITY}

We discuss here some important direct consequences of the previous estimates with the intention of applying them to quasiregular mappings.

5.1. $L^{p}$-integrability. Let us recall that every $K$-quasiregular mapping $f: \Omega \rightarrow \boldsymbol{R}^{n}$ is assumed to belong to $W_{n, \text { loc }}^{1}(\Omega)$. Lemma 3.2 shows that the first generalized derivatives of $f$ satisfy a weak inverse Hölder inequality (3.7). Now, on the basis of Theorem 4.2 we can prove that $f$ actually belongs to $W_{p, \text { loc }}^{1}(\Omega)$ with an exponent $p$ strictly greater than $n$. After these remarks we can formulate the following precise result.

Theorem 5.1. Let $f: \Omega \rightarrow \boldsymbol{R}^{n}$ be a K-quasiregular mapping. Then there exists $p=p(n, K)>n$ depending only on $n$ and $K$ such that $f \in W_{p, 1 \mathrm{oc}}^{1}(\Omega)$. Moreover, for every compact subset $F \subset \Omega$ the estimate

$$
\left(\int_{F}|D f(x)|^{p} d x\right)^{1 / p} \leqq \frac{C(n, p, K)}{[\operatorname{dist}(F, \partial \Omega)]^{1-n / p}}\left(\int_{Q}|D f(x)|^{n} d x\right)^{1 / n}
$$

holds with $C(n, p, K)$ independent of $f, F$ and $\Omega$.

Proof. A local estimate immediately follows from (3.7) and Theorem 4.2 so that for every subcube $Q \subset 2 Q \subset \Omega$ we have

$$
\left(f_{Q}|D f(x)|^{p} d x\right)^{1 / p} \leqq C\left(f_{Q}|D f(x)|^{n} d x\right)^{1 / n},
$$

where $C$ depends on $n, p$ and $K$. This reads equivalently as follows:

$$
\int_{Q}|D f(x)|^{p} d x \leqq C(\operatorname{diam} Q)^{n-p}\left(\int_{2 Q}|D f(x)|^{n} d x\right)^{p / n} .
$$

To go further, we shall consider the partition of $\boldsymbol{R}^{n}$ into closed congruent cubes with sides parallel to the axes and such that the interiors of two cubes of the partition are disjoint. The collection $\mathscr{M}$ of these cubes will be called a mesh of cubes in $\boldsymbol{R}^{n}$. The diameter - diam $\mathscr{M}$ - of the mesh is defined as the common diameter of cubes in $\mathscr{M}$. Let $\operatorname{diam} \mathscr{M}=(1 / 2) \operatorname{dist}(F, \partial \Omega)$. Then for each cube $Q \in \mathscr{M}$ which touches the set $F$ the double cube $2 Q$ is contained in $\Omega$. Therefore it follows 
from (5.3) that

$$
\begin{gathered}
\int_{F}|D f|^{p} \leqq \sum_{\substack{Q \in \mathcal{M} \\
Q \cap F \neq \Phi}} \int_{Q}|D f|^{p} \leqq 2^{p-n} C[\operatorname{dist}(F, \partial \Omega)]^{n-p} \sum_{\substack{Q \in \mathcal{M} \\
Q \cap F \neq \sigma}}\left(\int_{2 Q}|D f|^{n}\right)^{p / n} \\
\leqq 2^{p-n} C[\operatorname{dist}(F, \partial \Omega)]^{n-p}\left(\sum_{\substack{Q \in \mathcal{M} \\
Q \cap F \neq \Phi}} \int_{2 Q}|D f|^{n}\right)^{p / n}, \text { since } p / n \geqq 1 .
\end{gathered}
$$

We observe that each point $x \in \boldsymbol{R}^{n}$ is contained in at most $3^{n}$ of the cubes from the family $2 \mathscr{M}=\{2 Q ; Q \in \mathscr{M}\}$ of double cubes. Hence

Finally we get

$$
\sum_{\substack{Q \in \mathcal{M} \\ Q \cap F \neq \Phi}} \int_{2 Q}|D f|^{n} \leqq 3^{n} \int_{\substack{\cup Q Q \\ Q \in \mathcal{M} \\ Q \cap \mathcal{M} \neq \boldsymbol{Q}}}|D f|^{n} \leqq 3^{n} \int_{\Omega}|D f|^{n} .
$$

$$
\int_{F}|D f|^{p} \leqq 2^{p-n} \cdot 3^{p} C[\operatorname{dist}(F, \partial \Omega)]^{n-p}\left(\int_{\Omega}|D f|^{n}\right)^{p / n},
$$

and the proof is finished.

\subsection{Hölder continuity.}

Theorem 5.2. Any K-quasiregular mapping $f: \Omega \rightarrow \boldsymbol{R}^{n}$ is Hölder continuous on any compact subdomain $F \subset \Omega$. The Hölder exponent $\alpha=\alpha(n, K)$ depends only on $n$ and the dilatation $K_{f}=K$ while the Hölder coefficient depends on $n, K$, the geometry of the sets $F, \Omega$ and on the norm $\left(\int_{\Omega}|D f|^{n}\right)^{1 / n}$

$$
|f(x)-f(y)| \leqq C(n, K, F, \Omega)\|D f\|_{n}|x-y|^{\alpha}
$$

for every $x, y \in F$.

Proof. Suppose first that $F=Q$ is a cube and $\Omega=2 Q$. By Sobolev's imbedding Lemma 1.7 and by the inequality (5.3) we get

$$
\begin{aligned}
& |f(x)-f(y)| \leqq C(n, p)|x-y|^{1-n / p}\left(\int_{Q}|D f|^{p}\right)^{1 / p} \\
& \leqq C(n, p, K)\left(\frac{|x-y|}{\operatorname{diam} Q}\right)^{1-n / p}\left(\int_{\Omega}|D f|^{n}\right)^{1 / n}
\end{aligned}
$$

for every $x, y \in Q$. This gives (5.4) with $\alpha=1-n / p>0$. The general case follows from this particular one by use of routine methods. We omit the details.

Theorem 5.2 is surely much weaker than the precise and deep result of F. Gehring (see [13]) and it has only qualitative value. However, even this result on the continuity of quasiregular mappings has fundamental consequences in the study of geometric properties of $Q R$ mappings. 
5.3. Differentiation of $Q R$ mappings. In this section we show how the differentiability properties of quasiregular mappings follow from $L^{p}$ estimates of derivatives of the mappings. In agreement with our approach we are not interested in the greatest generality. Let $f: \Omega \rightarrow \boldsymbol{R}^{n}$ be a mapping and let $x_{0} \in \Omega$. We say that $f$ is differentiable at $x_{0}$ if there exists a linear map $L: \boldsymbol{R}^{n} \rightarrow \boldsymbol{R}^{n}$ such that

$$
f\left(x_{0}+h\right)=f\left(x_{0}\right)+L h+o(|h|)
$$

as the vector $h \in \boldsymbol{R}^{n}$ tends to zero. We call $L$ the differential of the map $f$ at the point $x_{0}$. Obviously $f$ is continuous at $x_{0}$ whenever it is differentiable. If $f$ is differentiable at $x_{0}$, then its partial derivatives at $x_{0}$ exist and the differential is the linear transformation identified with the Jacobi matrix $D f\left(x_{0}\right)$, but the converse is not true. It can be shown that there are mappings in the Sobolev space $W_{p, \text { loc }}^{1}(\Omega)$ with $p \leqq n$ not admitting differentials at any point of $\Omega$. Nevertheless for $p>n$ we have [11]

Proposition 5.1. Any map $f \in W_{p, \mathrm{loc}}^{\mathrm{1}}(\Omega)$ with $p>n$ is differentiable at almost every point $x \in \Omega$ and its differential is equal to $D f\left(x_{0}\right)$ the generalized Jacobi matrix.

Proof follows from the Lebesgue theorem. Indeed, since $D f \in L_{\text {loc }}^{p}(\Omega)$, the Lebesgue points of $D f$ form a subset $\Omega^{\prime}$ of full measure in $\Omega$ (see (1.7)). For each $x_{0} \in \Omega^{\prime}$ we have

$$
\lim _{r \rightarrow 0} f_{B\left(x_{0}, r\right)}\left|D f(y)-D f\left(x_{0}\right)\right|^{p} d y=0 .
$$

Let us put $g(x)=f(x)-f\left(x_{0}\right)-D f\left(x_{0}\right)\left(x-x_{0}\right)$; then $g\left(x_{0}\right)=0, g \in W_{p, \text { loc }}^{1}(\Omega)$ and $D g(x)=D f(x)-D f\left(x_{0}\right)$. By Sobolev's inequality (5.5) we get

$$
\begin{gathered}
\left|f(x)-f\left(x_{0}\right)-D f\left(x_{0}\right)\left(x-x_{0}\right)\right|=\left|g(x)-g\left(x_{0}\right)\right| \\
\leqq C(n, p) r\left(\int_{B\left(x_{0}, r\right)}|D g|^{p}\right)^{1 / p}=C(n, p) r\left(\int_{B\left(x_{0}, r\right)}\left|D f(y)-D f\left(x_{0}\right)\right|^{p} d y\right)^{1 / p}=o(r)
\end{gathered}
$$

for $\left|x-x_{0}\right| \leqq r$. This implies (5.6) with $L=D f\left(x_{0}\right)$. In view of Theorem 5.1 we have Theorem 5.3. A quasiregular map is differentiable at almost every point.

\section{VARIATIONAL INTEGRALS}

6.1. A special divergence type equation. To move further in our study of analytical properties of quasiconformal and quasiregular mappings, we shall need some particular properties of weak solutions of divergence equations

$$
\operatorname{div} A(x, \nabla u)=0
$$


with $A(x, \xi)$-the function from $\Omega \times \boldsymbol{R}^{n}$ into $\boldsymbol{R}^{n}$ of the form

$$
A(x, \xi)=\frac{n}{2}\langle G(x) \xi, \xi\rangle^{(n-2) / 2} G(x) \xi,
$$

where $G=G(x)$ is a symmetric positive definite $n \times n$ matrix-valued function with measurable entries defined in a domain $\Omega \subset \boldsymbol{R}^{n}$ and satisfying the uniform estimate

$$
\alpha^{2}|\xi|^{2} \leqq\langle G(x) \xi, \xi\rangle \leqq \beta^{2}|\xi|^{2}
$$

for almost all $x \in \Omega$ and all $\xi \in \boldsymbol{R}^{n}$ with some constants $\alpha, \beta, 0<\alpha^{2} \leqq \beta^{2}$.

The equation (6.1) is obviously the Euler-Lagrange equation for the functional

$$
I(u, \Omega)=\int_{\Omega} F(x, \nabla u) d x \text { with } F(x, \xi)=\langle G(x) \xi, \xi\rangle^{n / 2} .
$$

The weak solutions of (6.1) and the minima of the functional $I(u, \Omega)$ are looked for in the Sobolev space $W_{n}^{1}(\Omega)$. This means that for any test function $\eta \in \mathscr{W}_{n}^{1}(\Omega)$ the integral identity

$$
\int_{\Omega}\langle A(x, \nabla u), \nabla \eta\rangle d x=0
$$

holds. In the terminology of Paragraph 1.6 it expresses the fact that the vector $A(x, \nabla u)$ is divergence free.

For convenience we collect some elementary properties of the integrand $F(x, \xi)$ and the vector functions $A(x, \xi)$ in

Lemma 6.1. For almost all $x \in \Omega$ and all $\xi, \zeta \in \boldsymbol{R}^{n}$ we have

$$
\begin{gathered}
\frac{n}{2} \alpha^{n}|\xi|^{n} \leqq\langle A(x, \xi), \xi\rangle=\frac{n}{2} F(x, \xi) \\
|\langle A(x, \xi), \zeta\rangle| \leqq \frac{n}{2} \beta^{n}|\xi|^{n-1}|\zeta| \\
\langle A(x, \xi)-A(x, \zeta), \xi-\zeta\rangle \geqq \frac{n \alpha^{n}}{4}|\xi-\zeta|^{2}\left(|\xi|^{n-2}+|\xi|^{n-2}\right) \geqq \frac{n}{2}\left(\frac{\alpha}{2}\right)^{n}|\xi-\zeta|^{n} .
\end{gathered}
$$

Proof. The properties (6.6) and (6.7) are obvious. To get (6.8) we remark that $G$ admits the representation $G=Q^{*} Q$. Then $A(x, \xi) \xi=(n / 2)|Q \xi|^{n-2} Q^{*} Q \xi$ and thus

$$
\begin{aligned}
& \langle A(x, \xi)-A(x, \zeta), \xi-\zeta\rangle=\frac{n}{2}\left\langle|Q \xi|^{n-2} Q \xi-|Q \zeta|^{n-2} Q \zeta, Q \xi-Q \zeta\right\rangle \\
= & \frac{n}{4}|Q \xi-Q \zeta|^{2}\left(|Q \xi|^{n-2}+|Q \zeta|^{n-2}\right)+\frac{n}{4}\left(|Q \xi|^{2}-|Q \zeta|^{2}\right)\left(|Q \xi|^{n-2}-|Q \zeta|^{n-2}\right) \\
\geqq & \frac{n}{4}|Q \xi-Q \zeta|^{2}\left(|Q \xi|^{n-2}+|Q \zeta|^{n-2}\right)=\frac{n}{4}\langle G(\xi-\zeta), \xi-\zeta\rangle\left[\langle G \xi, \xi\rangle^{(n-2) / 2}\right. \\
& \left.+\langle G \zeta, \zeta\rangle^{(n-2) / 2}\right] \geqq \frac{n}{4} \alpha^{n}|\xi-\zeta|^{2}\left(|\xi|^{n-2}+|\zeta|^{n-2}\right) \geqq \frac{n}{2}\left(\frac{\alpha}{2}\right)^{n}|\xi-\zeta|^{n} .
\end{aligned}
$$


6.2. The function $u=-\ln |f|$. Suppose that $f: \Omega \rightarrow \boldsymbol{R}^{n} \backslash\{0\}$ is a quasiregular mapping, i.e., $f \in W_{n . \text { loc }}^{1}(\Omega)$ and

$$
|D f(x)|^{n} \leqq K J_{f}(x) \text { for almost every } \quad x \in \Omega .
$$

We define the matrix-valued function

$$
G^{-1}(x)= \begin{cases}\frac{D^{*} f(x) D f(x)}{J_{f}(x)^{2 / n}} & \text { if } D f(x) \text { exists and } J_{f}(x) \neq 0 \\ I & \text { otherwise. }\end{cases}
$$

By (6.9) we see that $G^{-1}(x)$ is defined everywhere in $\Omega$ as a symmetric positive definite $n \times n$ matrix such that $\operatorname{det} G^{-1}(x) \equiv 1$ and

$$
|\xi|^{2} \leqq\left\langle G^{-1}(x) \xi, \xi\right\rangle \leqq K^{2 / n}|\xi|^{2}
$$

Hence the inverse matrix, denoted by $G(x)$, satisfies

$$
K^{-2 / n}|\xi|^{2} \leqq\langle G \xi, \xi\rangle \leqq|\xi|^{2},
$$

which corresponds to the assumption (6.3) with $\alpha=K^{-1 / n}$ and $\beta=1$. It follows directly from the definition that for almost all $x \in \Omega$

$$
\left(J_{f}(x)\right)^{(n-2), n} G(x) D^{*} f(x)=\operatorname{adj} f(x), \text { and } \quad D f(x) G(x) D^{*} f(x)=J_{f}^{2 / n}(x) I,
$$

where $\operatorname{adj} f(x)$ is the adjugate matrix (see 1.35).

Notice that (6.12) follows from (6.10) if $J_{f}(x) \neq 0$. In the case $J_{f}(x)=0$ both the left and the right hand side of (6.12) vanish because $D f(x)=0$; see (6.9).

Let us now consider the function $u(x)=-\ln |f(x)|$. We easily verify that

$$
\nabla u(x)=-D^{*} f(x) \frac{f(x)}{|f(x)|^{2}}
$$

Hence

and

$$
\begin{gathered}
\langle G(x) \nabla u, \nabla u\rangle=|f|^{-4}\left\langle\left(G D^{*} f\right) f,\left(D^{*} f\right) f\right\rangle=|f|^{-4}\left\langle\left(D f G D^{*} f\right) f, f\right\rangle \\
=J_{f}^{2 / n}|f|^{-4}\langle f, f\rangle=J_{f}^{2 / n}|f|^{-2}
\end{gathered}
$$

$$
G(x) \nabla u=-|f|^{2}\left(G D^{*} f\right) f .
$$

Therefore $\langle G(x) \nabla u, \nabla u\rangle^{(n-2) / 2} G(x) \nabla u=-|f|^{-n}\left(J_{f}^{(n-2) / n} G D^{*} f\right) f=-(\operatorname{adj} f(x)) f /|f|^{n}$. On the other hand, we know that the vector $(\operatorname{adj} f) f /|f|^{n}$ is divergence free (see Corollary 1.1). Thus we have proved

Lemma 6.2. For any quasiregular mapping $f: \Omega \rightarrow \boldsymbol{R}^{n}-\{0\}$ the function $u=-\ln |f|$ is a weak solution of the equation (6.1) with $G(x)$ satisfying (6.11). 


\subsection{Basic estimates of weak solutions.}

Proposition 6.1. Let $u$ be a weak solution of (6.1). Then for every test function $\varphi \in C_{0}(\Omega), \varphi \geqq 0$ with bounded gradient $\nabla \varphi(x)$ and for every constant $c$ the following inequality holds:

$$
\left(\int_{\Omega} \varphi^{n}(x)|\nabla u(x)|^{n} d x\right)^{1 / n} \leqq \frac{n \beta^{n}}{\alpha^{n}}\left(\int_{\Omega}|\nabla \varphi(x)|^{n}|u(x)-c|^{n} d x\right)^{1 / n} .
$$

If the weak solution $u$ is positive in $\Omega$, then the function $v=\ln u$ belongs to $W_{n, \operatorname{loc}}^{1}(\Omega)$ and satisfies the inequality

$$
\left(\int_{\Omega}|\varphi(x)|^{n}|\nabla v(x)|^{n} d x\right)^{1 / n} \leqq \frac{\beta^{n}}{\alpha^{n}} \frac{n}{n-1}\left(\int_{\Omega}|\nabla \varphi(x)|^{n} d x\right)^{1 / n}
$$

for every $\varphi \in \stackrel{\circ}{W}_{n}^{1}(\Omega)$.

Remark. Since $u(x)+\varepsilon$ is also a solution of the equation (6.1) for every constant $\varepsilon$, we may assume for the proof of (6.14) without loss of generality that $u$ is strictly positive, i.e., $u(x) \geqq \varepsilon>0$ for some $\varepsilon$.

Proof. In both cases we use the integral identity (6.5) and a proper choice of the test function $\eta$. To get (6.13) we take $\eta(x)=\varphi^{n}(x)(u(x)-c) \in \dot{W}_{n}^{1}(\Omega)$. Then $\nabla \eta=n \varphi^{n-1}(u-c) \nabla \varphi+\varphi^{n} \nabla u$ and

$$
\int_{\Omega} \varphi^{n}\langle A(x, \nabla u), \nabla u\rangle d x=-n \int_{\Omega} \varphi^{n-1}(u-c)\langle A(x, \nabla u), \nabla \varphi\rangle d x
$$

Hence, in view of (6.6) and (6.7) and by the Hölder inequality

$$
\begin{aligned}
& \frac{n \alpha^{n}}{2} \int_{\Omega} \varphi^{n}|u|^{n} \leqq n \frac{n \beta^{n}}{2} \int_{\Omega} \varphi^{n-1}|u-c||\nabla \varphi||\nabla u|^{n-1} \\
& \leqq \frac{n^{2} \beta^{n}}{2}\left(\int_{\Omega} \varphi^{n}|\nabla u|^{n}\right)^{(n-1) / n}\left(\int_{\Omega}|\nabla \varphi|^{n}|u-c|^{n}\right)^{1 / n}
\end{aligned}
$$

and (6.13) follows. To get (6.14) we substitute $\eta=\varphi^{n} / u^{n-1} \in \dot{W}_{n}^{1}(\Omega)$. Then

$$
\nabla \eta=\frac{n \varphi^{n-1} \nabla \varphi}{u^{n-1}}-(n-1) \frac{\varphi^{n} \nabla v}{u^{n-1}} \quad \text { since } \quad u \nabla v=\nabla u
$$

and

$$
(n-1) \int_{\Omega} \varphi^{n}\langle A(x, \nabla v), \nabla v\rangle d x=n \int_{\Omega} \varphi^{n-1}\langle A(x, \nabla v), \nabla \varphi\rangle d x
$$


Hence by (6.6), (6.7) and the Hölder inequality

$$
\begin{gathered}
(n-1) \frac{n \alpha^{n}}{2} \int_{\Omega} \varphi^{n}|\nabla v|^{n} \leqq \frac{n^{2} \beta^{n}}{2} \int_{\Omega} \varphi^{n-1}|\nabla \varphi||\nabla v|^{n-1} \\
\leqq \frac{n^{2} \beta^{n}}{2}\left(\int_{\Omega} \varphi^{n}|\nabla v|^{n}\right)^{(n-1) ! n}\left(\int_{\Omega}|\nabla \varphi|^{n}\right)^{1 / n},
\end{gathered}
$$

and (6.14) follows.

As a consequence of estimate (6.13) we get

Corollary 6.1. If the ball $B\left(x_{0}, 2 r\right) \subset \Omega$, then the Caccioppoli type inequality

$$
\left(\int_{B\left(x_{0}, r\right)}|\nabla u(x)|^{n} d x\right)^{1 / n} \leqq \frac{n \beta^{n}}{\alpha^{n} r}\left(\int_{B\left(x_{0}, 2 r\right)}|u(x)-c|^{n} d x\right)^{2 / n}
$$

holds.

Notice that $(6.15)$ is valid with $B$ replaced by a cube $Q$ with another coefficient which can easily be evaluated.

Proof. The inequality (6.15) arises when we put $\varphi$ in (6.13) such that $\varphi(x)=1$ in $B\left(x_{0}, r\right)$ and $|\nabla \varphi(x)| \leqq 1 / r$ in $B(x, 2 r)$. Taking $c=f_{B\left(x_{0}, 2 r\right)} u(y) d y$ and using the local Poincaré-Sobolev inequality (see 1.24)

$$
\left(f_{B\left(x_{0}, 2 r\right)}\left|u(y)-\int_{B\left(x_{0}, 2 r\right)} u\right|^{n} d y\right)^{1 / n} \leqq C(n) r\left(f_{B\left(x_{0}, 2 r\right)}|\nabla u(y)|^{n / 2} d y\right)^{2 / n}
$$

we obtain the weak inverse Hölder inequality

$$
\left(f_{B\left(x_{0}, r\right)}|\nabla u|^{n}\right)^{1 / n} \leqq c(n, \alpha, \beta)\left(f_{B\left(x_{0}, 2 r\right)}|\nabla u|^{n / 2}\right)^{2 / n} .
$$

In view of Paragraph 4.3 we have the important

Corollary 6.2. Every weak solution $u \in W_{n}{ }^{1}(\Omega)$ of (6.1) actually belongs to some $W_{p, \text { loc }}^{1}(\Omega)$ with $p>n$. In particular, by Sobolev's imbedding theorem, weak solutions of (6.1) and local minima of the functional (6.4) are Hölder continuous in $\Omega$.

6.4. Conformal capacity. We recall now the concept of the $m$-capacity of the pair $(F, \Omega)$, where $F$ is a compact subset of $\Omega$.

Definition 6.1. The m-capacity $\operatorname{Cap}_{m}(F, \Omega)$ of the pair $(F, \Omega)$ is defined as

$$
\operatorname{Cap}_{m}(F, \Omega)=\inf _{\varphi \in \dot{W}_{m}^{1}(\Omega)} \int_{\Omega}|\nabla \varphi|^{m},\left.\quad \varphi\right|_{F} \geqq 1, \quad m \geqq 1
$$

Obviously

$$
\operatorname{Cap}_{m}(F, \Omega) \leqq \operatorname{Cap}_{m}\left(F^{\prime}, \Omega^{\prime}\right) \quad \text { if } \quad F \subset F^{\prime} \subset \subset \Omega^{\prime} \subset \Omega .
$$

The $n$-capacity will also be called the conformal capacity. The inequality (6.14) can be used to estimate the $L^{n}(F)$ norm of the gradient of $v=-\ln u$, where $u$ is 
a positive weak solution of divergence equation (6.1), in terms of conformal capacity of the pair $(F, \Omega)$.

Corollary 6.3. If $v=\ln u$ and $u>0$ is a weak solution of (6.1), then for every compact subset $F \subset \Omega$ the inequality

$$
\left(\int_{F}|\nabla v|^{n}\right)^{1 / n} \leqq \frac{n}{n-1} \frac{\beta^{n}}{\alpha^{n}}\left[\operatorname{Cap}_{m}(F, \Omega)\right]^{1 / n}
$$

holds.

In particular, if $B\left(x_{0}, r\right) \subset B\left(x_{0}, R\right) \subset \Omega$, then

$$
\int_{B\left(x_{0}, r\right)}|\nabla v|^{n} \leqq\left(\frac{n \beta^{n}}{(n-1) \alpha^{n}}\right)^{n} \frac{\omega_{n}}{\ln ^{n-1}(R / r)},
$$

where $\omega_{n}$ is the volume of the unit sphere in $\boldsymbol{R}^{n}$.

Proof. The inequality (6.18) follows if we take the infimum on the right-hand side of (6.14) over all admissible $\varphi, \varphi \geqq 1$ on $F$. The inequality (6.19) is obvious if we assume the conformal capacity of the pair $\left(\overline{B\left(x_{0}, r\right)}, B\left(x_{0}, R\right)\right), R>n$ known. A simple derivation of this fact will follow from some considerations below; see the formula (6.24).

6.4. Existence and uniqueness. So far we did not use the important property (6.8) of the function $A(x, \xi)$. It expresses the fact that the "form" $\langle A(x, \xi)-A(x, \zeta)$, $\xi-\zeta\rangle$ is positive definite and has familiar consequences of monotonicity. Specially (6.8) implies that the non-linear Dirichlet problem

$$
\begin{gathered}
\operatorname{div} A(x, \nabla u)=0, \quad x \in \Omega \\
u-\varphi \in \stackrel{\circ}{n}_{n}^{1}(\Omega)
\end{gathered}
$$

for a given $\varphi \in W_{n}^{1}(\Omega)$ has a unique weak solution $u$ in $W_{n}^{1}(\Omega)$. Moreover, the maximum and minimum principle (comparison principle) for weak solutions of (6.20-21) holds.

Both above facts are well known and their proofs are completely standard.

We discuss them for completeness. The solution of the Dirichlet problem is obtained by minimization of the functional $I(u, \Omega)$ over the subset of $W_{n}^{1}(\Omega)$ of functions satisfying the boundary condition (6.21). Let $u_{j}$ be an arbitrary minimizing sequence for $I(u, \Omega)$ such that $u_{j}-\varphi \in \dot{W}_{n}^{1}(\Omega)$, i.e.,

$$
I_{\min }=\inf _{\substack{u=W_{n}^{1}(\Omega) \\ u-\varphi \in W_{n}^{1}(\Omega)}} \int_{\Omega}\langle G(x) \nabla u, \nabla u\rangle^{n / 2} d x=\lim _{j \rightarrow \infty} \int_{\Omega}\left\langle G(x) \nabla u_{j}, \nabla u_{j}\right\rangle^{n / 2} d x .
$$

By (6.6) we see that the norms $\left\|u_{j}\right\|_{L^{n}(\Omega)}$ are uniformly bounded. Hence the func. tions $u_{j}-\varphi \in \stackrel{\circ}{W}_{n}^{1}(\Omega)$ are uniformly bounded in $\stackrel{\circ}{W}_{n}^{1}(\Omega)$ and consequently the set $\left\{u_{j}\right\}$ is weakly compact in $W_{n}^{1}(\Omega)$. Any weak limit $u=\lim _{k \rightarrow \infty} u_{j_{k}}$ is the required solution of (6.20) and (6.21) because it minimizes the integral $I(u, \Omega)$. 
In fact, let $\zeta(x)=\langle G(x) \nabla u(x), \nabla u(x)\rangle^{(n-2) / 2} \nabla u(x) \in L^{n /(n-1)}(\Omega)$. Then

$$
\begin{gathered}
\int_{\Omega}\langle G(x) \nabla u(x), \nabla u(x)\rangle^{n / 2} d x=\int_{\Omega}\langle G(x) \nabla u(x), \zeta(x)\rangle d x=\lim _{k \rightarrow \infty} \int_{\Omega}\left\langle G \nabla u_{j_{k}}, \zeta\right\rangle \\
\leqq \varlimsup_{k \rightarrow \infty} \int_{\Omega}\left\langle G \nabla u_{j_{k}}, \nabla u_{j_{k}}\right\rangle^{1 / 2}\langle G \zeta, \zeta\rangle^{1 / 2} \\
\leqq \varlimsup_{k \rightarrow \infty}\left(\int_{\Omega}\left\langle G \nabla u_{j_{k}}, \nabla u_{j_{k}}\right\rangle^{n / 2}\right)^{1 / n}\left(\int_{\Omega}\langle G \zeta, \zeta\rangle^{n /(2(n-1))}\right)^{(n-1) / n} \\
=I_{\min }^{1 / n}\left(\int_{\Omega}\langle G(x) \nabla u(x), \nabla u(x)\rangle^{n / 2} d x\right)^{(n-1) / n} .
\end{gathered}
$$

Hence $\int_{\Omega}\langle G(x) \nabla u(x), \nabla u(x)\rangle^{n / 2} d x \leqq I_{\min }$.

But in view of the definition of $I_{\min }$ the function $u$ must equalize the last inequality. To prove uniqueness we assume by contradiction that $u_{1}$ and $u_{2}$ solve the problem $(6.20),(6.21)$ and $u_{1} \neq u_{2}$ as elements in $W_{n}^{1}(\Omega)$. Therefore

$$
\int_{\Omega}\left\langle A\left(x, \nabla u_{1}\right)-A\left(x, \nabla u_{2}\right), \nabla \eta\right\rangle d x=0
$$

for any $\eta \in \dot{W}_{n}^{1}(\Omega)$. Since $u_{1}$ and $u_{2}$ satisfy the same boundary conditions we are justified to substitute $\eta(x)=u_{1}-u_{2} \in \stackrel{\circ}{W}_{n}^{1}(\Omega)$ getting

$$
\int_{\Omega}\left\langle A\left(x, \nabla u_{1}\right)-A\left(x, \nabla u_{2}\right), \nabla u_{1}-\nabla u_{2}\right\rangle=0
$$

and by (6.8) $\int_{\Omega}\left|\nabla\left(u_{1}-u_{2}\right)\right|^{n} \leqq 0, \nabla\left(u_{1}-u_{2}\right)=0, u_{1} \equiv u_{2}$ almost everywhere in $\Omega$. So we reached the contradiction.

Suppose now that we are given two solutions $u_{1}$ and $u_{2}$ such that $u_{1} \geqq u_{2}$ on $\partial \Omega$. This means that the function $\eta=\min \left(u_{1}-u_{2}, 0\right)$ belongs to $\stackrel{\circ}{W}_{n}^{1}(\Omega)$ and thus can be used as a test function in the identity (6.22), which in view of (6.8) implies that $\int_{Q}|\nabla \eta|^{n} \leqq 0$ or $\eta \equiv 0$, i.e., $u_{1} \geqq u_{2}$ almost everywhere in $\Omega$. This last inequality holds everywhere in $\Omega$ on account of the continuity property of the solutions $u_{1}$ and $u_{2}$. This is exactly the assertion of the comparison principle. In particular, letting $u_{1}$ or $u_{2}$ to be constants we immediately get the maximum and the minimum principles.

As a consequence of the above remarks we get:

Corollary 6.4. The conformal capacity $\operatorname{Cap}_{n}(F, \Omega)$ can always be calculated from the unique solution to the weak Dirichlet problem

$$
\begin{cases}\operatorname{div}\left|\nabla u_{0}\right|^{n-2} \nabla u_{0}=0 & \text { in } \Omega \backslash F \\ u_{0}=0 & \text { on } \partial \Omega \\ u_{0}=1 & \text { on } \partial F .\end{cases}
$$

When applied to $F=\overline{B\left(x_{0}, r\right)} \subset B\left(x_{0}, R\right)=\Omega, r<R$, this corollary gives the exact expression for the capacity of the pair $\left(\overline{B\left(x_{0}, r\right)}, B\left(x_{0}, R\right)\right)$ used in the deri- 
vation of (6.19), namely,

and

$$
u_{0}(x)=\frac{\ln \left(\left|x-x_{0}\right| / R\right)}{\ln (r / R)}, \quad \nabla u_{0}(x)=\frac{x-x_{0}}{\left|x-x_{0}\right|^{2} \ln (r / R)}
$$

$$
\operatorname{Cap}_{n}\left(\overline{B\left(x_{0}, r\right)}, B\left(x_{0}, R\right)\right)=\int_{r<\left|x-x_{0}\right|<R}\left|\nabla u_{0}(x)\right|^{u} d x=\frac{\omega_{n}}{\ln ^{n-1}(R / r)} .
$$

6.5. A weak form of Harnack's inequality. We shall show now that the basic estimate (6.19) implies a principle of the Harnack type for non-negative weak solutions of the class $W_{n}^{1}(\Omega)$ of divergence equation (6.1). To achieve that we need a sharpening of Sobolev's imbedding inequality (1.27) in the borderline case $W_{n}^{1}(\Omega)$.

Proposition 6.2. (Weak local Harnack's principle). Let $u$ be a non-negative function of the class $W_{n}^{1}(\Omega)$ such that for every cube $Q \subset 3 Q \subset \Omega$

$$
\int_{3 Q}|\nabla \ln u|^{n} \leqq \frac{v(n)}{3 n},
$$

where $v(n)$ is the constant appearing in Lemma 1.6. Then

$$
f_{3 Q} u^{n} \leqq 2 f_{Q} u^{n}
$$

Proof. Apply (1.27) to $\sigma=1 / 3$ and $v=\ln u$; then

$$
\int_{3 Q} e^{n\left|\ln u-(\ln u)_{Q}\right|} \leqq 2
$$

or

$$
\int_{3 Q} u^{n} \leqq 2 e^{f \ln u^{n}} \leqq 2 f_{Q} e^{\ln u^{n}}=2 f_{Q} u^{n}
$$

by Jensen's inequality (1.15) applied to $\emptyset(t)=e^{t}$. By iteration the inequality (6.26) is immediately globalized as follows:

Proposition 6.3. Let $\left\{Q_{j}\right\}, j=1,2, \ldots, N$ be a sequence of parallel congruent cubes $Q_{j} \subset \Omega$ such that $3 Q_{j} \subset \Omega, Q_{j} \cap Q_{j+1} \neq \emptyset$ for $j=1,2, \ldots, N-1$ and $j=1,2, \ldots, N$ (6.26) holds for each $Q_{j}$. Then

$$
\int_{Q_{i}} u^{n} \leqq\left(2 \cdot 3^{n}\right)^{N-1} \int_{Q_{j}} u^{n} \quad \text { for } \quad i, j=1,2, \ldots, N .
$$

Proof. For $N=2$ we have

$$
\int_{Q_{1}} u^{n} \leqq \int_{3 Q_{2}} u^{n} \leqq 2 \cdot 3^{n} \int_{Q_{2}} u^{n}
$$

since obviously $Q_{1} \subset 3 Q_{2}$ if $Q_{1} \cap Q_{2} \neq \emptyset$. The general case follows by iteration of the above inequality. 
Combining (6.27) with the Caccioppoli type inequality (6.15) we can easily prove the following version of the Harnack type inequality:

Theorem 6.1. For any compact subsets $F_{1}$ and $F_{2}$ of a domain $\Omega$, Int $F_{2} \neq \emptyset$ and any divergence type equation (6.1) satisfying the condition (6.3) there exists a constant $C=C\left(n, \alpha, \beta, F_{1}, F_{2}, \Omega\right)$ such that for any non-negative solution $u$ of (6.1) in $\Omega$ the following inequality holds:

$$
\int_{F_{1}}|\nabla u(x)|^{n} d x \leqq C \int_{F_{2}}|u(x)|^{n} d x .
$$

Proof. We obviously may assume that $u$ is positive. Then we can examine the function $v=\ln u$. For $F_{1}$ and $F_{2}$ given we choose a covering of $F_{1} \cup F_{2}$ by a finite number of coordinate parallel cubes $Q_{1}, \ldots, Q_{N}$ with fixed sidelength $\varepsilon$ small enough to satisfy the following conditions:

a) $2 Q_{1} \subset F_{2}$

b) $6 Q_{i} \subset \Omega \quad$ for $i=1,2, \ldots, N$

c) $Q_{i} \cap Q_{i+1} \neq \emptyset$ for $i=1,2, \ldots, N-1$

d) $\int_{6 Q_{j}}|\nabla \ln u|^{n} \leqq \frac{v(n)}{3 n}, \quad j=1,2, \ldots, N$ (see 6.25).

Such a choice can be made with the numbers $N$ and $\varepsilon$ depending only on $n, \alpha, \beta$ and the geometry of the sets $F_{1}, F_{2}, \Omega$. In fact, taking into account the uniform estimate (6.19) we see that the condition d) and also the conditions a) and b) are guaranteed by a choice of sufficiently small $\varepsilon$ i.e., the size of the cubes $Q_{i}$ covering the compact subset $F_{1} \cup F_{2}$. In order to have c) it is enough to take sufficiently many of such cubes, say $N$.

Notice that the cubes $Q_{1}, \ldots, Q_{N}$ can be chosen for example from a family of cubes obtained by sufficiently small regular division of $\boldsymbol{R}^{\boldsymbol{n}}$. Condition d) in view of Proposition 6.3 implies

$$
\int_{2 Q_{i}} u^{n} \leqq\left(2 \cdot 3^{n}\right)^{N-1} \int_{2 Q_{1}} u^{n} \leqq\left(2 \cdot 3^{n}\right)^{N-1} \int_{F_{2}} u^{n} \text { for } i=1,2, \ldots, N .
$$

Now the Caccioppoli type estimate (6.15) yields

$$
\int_{Q_{i}}\left|\nabla u^{n}\right| \leqq \frac{C(n, \alpha, \beta)}{\left(\operatorname{diam} Q_{i}\right)^{n}} \int_{2 Q_{i}} u^{n} \leqq \frac{C(n, \alpha, \beta, N)}{\varepsilon^{n}} \int_{F_{2}} u^{n} \text { for each } i=1, \ldots, N .
$$

Since the cubes $Q_{i}$ cover $F_{1}$, the inequality (6.28) with $C=N \varepsilon^{-n} C(n, \alpha, \beta, N)=$ $C\left(n, \alpha, \beta, F_{1}, F_{2}, \Omega\right)$ follows. 


\section{CAPACITY AND HAUSDORFF MEASURE ESTIMATES FOR QUASIREGULAR MAPPINGS}

Now we have at our disposal all tools needed to prove two important theorems.

\subsection{A capacity estimate.}

Theorem 7.1. Let $f: \Omega \rightarrow \boldsymbol{R}^{n}$ be $K$-quasiregular, $\left.f \in W_{n}^{1}(\Omega), f \neq 0{ }^{*}\right)$ Assume $0<M=\sup _{x \in \Omega}|f(x)|<\infty$. Let $E$ be a compact subset of $\Omega$ and denote $E_{t}=$ $\{x \in E ;|f(x)| \leqq t\}$. Then there exists a constant $C$ depending on $n, K, E, \Omega$ and $f$, but not on $t$, such that

$$
\operatorname{Cap}_{n}\left(E_{t}, \Omega\right) \leqq C \ln ^{1-n} \frac{M}{t} \text { for } t \leqq \frac{M}{2} .
$$

Proof. We fix two open subsets $\Omega_{1}$ and $\Omega_{2}$ of $\Omega$, such that $E \subset \subset \Omega_{1} \subset \subset \Omega_{2} \subset \subset \Omega$, and put $F_{1}=\bar{\Omega}_{2}-\Omega_{1}$. Since $f$ is continuous and $f \not \equiv 0$, there exists a compact subset $F_{2}$ containing an open cube such that

$$
\inf _{x \in F_{2}}|f(x)|=t_{0}>\frac{M}{2}>0 .
$$

Obviously $F_{1} \subset \Omega-E_{t} \equiv \Omega_{t}$ for all $t$.

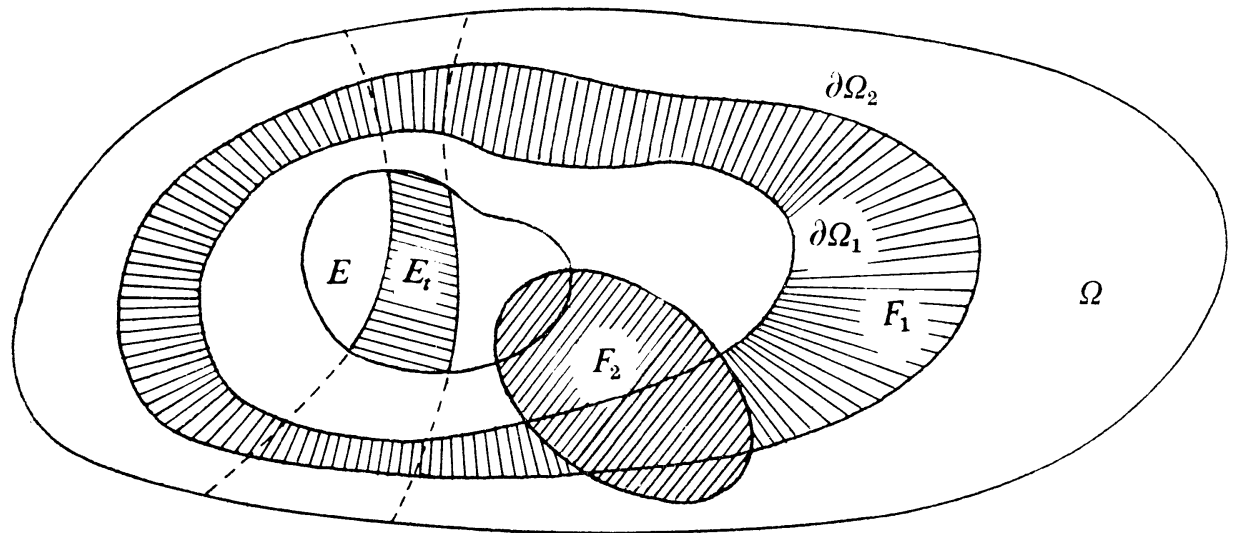

The essential idea in the proof of the estimate (7.1), due to Rešetnjak [30], is the fact that the function $u(x)=\ln (M /|f(x)|)$ is a weak solution of the equation (6.1) (see Lemma 6.2) and the use of some barriers for $u$, constructed as weak solutions of the same equation. The crucial property of $A(x, \xi)$ in this construction is that it

*) For simplicity we assume that $f$ is defined and continuous on $\bar{\Omega}$. 
homogeneous in $\xi ; A(x, \lambda \xi)=\lambda^{n-1} A(x, \xi)$ for $\lambda \in \boldsymbol{R}^{1}$. Definition 6.1 allows us to take into consideration functions $\omega \in W_{n}^{1}\left(\Omega_{t}\right)$ satisfying the boundary conditions

$$
\varphi=0 \quad \text { on } \partial \Omega \text { and } \varphi=1 \text { on } \partial E_{t} .
$$

In particular, we can take as $\varphi$ the weak solution $\varphi=u_{t}$ of the Dirichlet problem

$$
\left\{\begin{array}{l}
\operatorname{div} A\left(x, \nabla u_{t}\right)=0 \quad \text { in } \Omega_{t} \\
u_{t}=0 \text { on } \partial \Omega, \quad u_{t}=1 \text { on } \partial E_{t} .
\end{array}\right.
$$

Then in view of (6.6) and (6.5) we shall have

$$
\operatorname{Cap}_{n}\left(E_{t}, \Omega\right) \leqq \int_{\Omega_{t}}\left|\nabla u_{t}\right|^{n} \leqq \frac{2}{n \alpha^{n}} \int_{\Omega_{t}}\left\langle A\left(x, \nabla u_{t}\right), \nabla u_{t}\right\rangle=\frac{2}{n \alpha^{n}} \int_{\Omega_{t}}\left\langle A\left(x, \nabla u_{t}\right), \nabla \eta\right\rangle
$$

for any function $\eta \in W_{n}^{1}\left(\omega_{t}\right)$ such that $\eta-u_{t} \in \dot{W}_{n}^{1}\left(\Omega_{t}\right)$. On the other hand, any function $\eta \in C^{1}(\Omega)$ such that

$$
\eta=0 \text { for } x \in \Omega-\Omega_{2}, \quad \eta=1 \text { for } x \in \Omega_{1}
$$

has this property if $t \leqq M / 2$. Therefore for any such $\eta$ we have $\nabla \eta(x) \equiv 0$ if $x \notin F_{1}$; thus by (6.7)

$$
\begin{aligned}
\operatorname{Cap}_{n}\left(E_{t}, \Omega\right) & \leqq \frac{2}{n \alpha^{n}} \int_{F_{1}}\left\langle A\left(x, \nabla u_{t}\right), \nabla \eta\right\rangle \leqq\left(\frac{\beta}{\alpha}\right)^{n} \int_{F_{1}}\left|\nabla u_{t}\right|^{n-1}|\nabla \eta| \\
& \leqq\left(\frac{\beta}{\alpha}\right)^{n}\left(\int_{F_{1}}\left|\nabla u_{t}\right|^{n}\right)^{(n-1) / n}\left(\int_{F_{1}}|\nabla \eta|^{n}\right)^{1 / n}
\end{aligned}
$$

or, since $\eta$ is arbitrary satisfying (7.5),

$$
\operatorname{Cap}_{n}\left(E_{t}, \Omega\right) \leqq\left(\frac{\beta}{\alpha}\right)^{n} \operatorname{Cap}_{n}\left(\bar{\Omega}_{1}, \Omega_{2}\right)\left(\int_{F_{1}}\left|\nabla u_{t}\right| n\right)^{(n-1) / n} .
$$

Now we use the Harnack inequality (6.28) to estimate the right-hand side in (7.6). Consider the function $\bar{u}_{t}(x)=u_{t}(x) \ln (M / t)$ in the domain $\Omega_{t}$. Both $\bar{u}_{t}(x)$ and $u(x)=\ln (M /|f(x)|)$ are weak solutions of the equation $\operatorname{div} A(x, \nabla u)=0$ in the open set $\{x \in \Omega ;|f(x)|>t\} \subset \Omega_{t}$ on the boundary of which we have $u(x) \geqq \bar{u}_{t}(x)$. In fact, by the conditions (7.4) and by the comparison principle (see Paragraph 6.4) $1 \geqq u_{t}(x) \geqq 0$ everywhere in $\Omega_{t}$; thus $\ln (M / t) \geqq \bar{u}_{t}(x) \geqq 0$ in $\Omega_{t}$. For every point $x$ from the boundary of $\{x \in \Omega ;|f(x)|>t\}$ we either have $|f(x)|=t, u(x)=\ln (M / t) \geqq$ $\bar{u}_{t}(x)$ or $x \in \partial \Omega$ and so $u(x)=\ln (M /|f(x)|) \geqq \ln (M / M)=0=\bar{u}_{t}(x)$.

Now by the comparison principle we conclude

$$
u(x) \geqq \bar{u}_{t}(x) \text { for } \quad x \in\{x \in \Omega ;|f(x)|>t\} .
$$

Since $t \leqq M / 2$, then $F_{2} \subset\{x \in \Omega ;|f(x)|>t\}$. In particular, for $x \in F_{2}$

$$
\bar{u}_{t}(x) \leqq u(x)=\ln \frac{M}{|f(x)|}<\ln \frac{M}{M / 2}=\ln 2 .
$$


Since both $F_{1}$ and $F_{2}$ are compact subsets of $\Omega_{t_{0}}$, the Harnack inequality (6.28) applied to the weak solution $\bar{u}_{t}$ of the equation $\operatorname{div}(x, \nabla u)=0$ in $\Omega_{t_{0}}$ gives

$$
\int_{F_{1}}\left|\nabla \bar{u}_{t}\right|^{n} \leqq \bar{C} \int_{F_{2}}\left|\bar{u}_{t}\right|^{n}<\bar{C} \ln ^{n} 2\left(\operatorname{mes} F_{2}\right)
$$

with the constant $\bar{C}$ independent of the solution $\bar{u}_{t}$ and, in particular, independent of the parameter $t \leqq M / 2$. Finally (7.6) and (7.8) imply (7.1) with $C=C(n, K, E, \Omega, f)$ In fact

$$
\operatorname{Cap}_{n}\left(E_{t}, \Omega\right) \leqq\left(\frac{\beta}{\alpha}\right)^{n} \operatorname{Cap}_{n}^{1 / n} \bar{\Omega}\left({ }_{1}, \Omega_{2}\right)\left(\int_{F_{1}}\left(\ln \frac{M}{t}\right)^{-n}\left|\nabla \bar{u}_{t}\right|^{n}\right)^{(n-1) / n} \leqq C \ln ^{1-n} \frac{M}{t} .
$$

Corollary 7.1. Let $f: \Omega \rightarrow \boldsymbol{R}^{n}$ be a quasiregular mapring, $f \not \equiv$ const. Then

$$
\operatorname{Cap}_{n}\left(f^{-1}(y)\right)=0 \quad \text { for every } \quad y \in \boldsymbol{R}^{n} .
$$

Here $f^{-1}(y)$ is the preimage of a point $y$ under the mapping $f$.

Remark. The equality (7.9) should be understood as follows:

$$
\operatorname{Cap}_{n}\left(E \cap f^{-1}(y), \Omega\right)=0 \text { for every compact } E \subset \Omega .
$$

Proof. If $y=0$, then (7.9) directly follows from Theorem 7.1 because

$$
\operatorname{Cap}_{n}\left(E \cap f^{-1}(y), \Omega\right) \leqq \operatorname{Cap}_{n}\left(E_{t}, \Omega\right) \leqq C \ln ^{1-n} \frac{M}{t} \quad \text { for each } \quad t \leqq \frac{M}{2}
$$

The full assertion is the consequence of this inequality applied to the shifted mappings.

Now, for a non-degenerate quasiregular mapping $f: \Omega \rightarrow \boldsymbol{R}^{n}$ we introduce the modul of continuity

$$
\omega\left(x_{0}, r\right)=\sup _{\left|x-x_{0}\right| \leqq r}\left|f(x)-f\left(x_{0}\right)\right|
$$

defined for each $x_{0} \in \Omega$ and $r \leqq(1 / 2) \operatorname{dist}\left(x_{0}, \partial \Omega\right)$.

Corollary 7.2. For each $x_{0} \in \Omega$ there exist an exponent $\lambda>0$ and a constant $C\left(x_{0}\right)$ such that

$$
\omega\left(x_{0}, r\right) \geqq C\left(x_{0}\right) r^{\lambda} \quad \text { for } \quad r \leqq(1 / 2) \operatorname{dist}\left(x_{0}, \partial \Omega\right) .
$$

In general, $\lambda$ and $C\left(x_{0}\right)$ may depend on the mapping $f$.

Proof. We assume for simplicity that $x_{0}=0, f\left(x_{0}\right)=0$. Fix a ball $E=\overline{B(0, R)} \subset \Omega$ and $r_{0}<R$ such that $M_{r}=\sup _{|x| \leqq_{r}}|f(x)| \leqq M / 2=(1 / 2) \sup _{x \in \Omega}|f(x)|$ for $r \leqq r_{0}$. Then, in view of (7.1) and the obvious inclusion $\overline{B(0, r)} \subset\left\{x \in B(0, R) ;|f(x)| \leqq M_{r}\right\}=$ $E_{M_{r}}$ we get

$$
\left.\operatorname{Cap}_{n}(\overline{B(0, r}), B(0, R)\right) \leqq \operatorname{Cap}_{n}\left(E_{M_{r}}, \Omega\right) \leqq C \ln ^{1-n} \frac{M}{M_{r}}
$$


or, in view of (6.24),

$$
\frac{\omega_{n}}{\ln ^{n-1}(R / r)} \leqq \frac{C}{\ln ^{n-1}\left(M / M_{r}\right)},
$$

where $C$ does not depend on $r \leqq r_{0}$. Hence

$$
\omega\left(x_{0}, r\right)=M_{r} \geqq M\left(\frac{r}{R}\right)^{\lambda}, \quad \lambda=\left(C / \omega_{n}\right)^{1 /(n-1)} .
$$

For $r_{0} \leqq r \leqq(1 / 2)$ dist $\left(x_{0}, \partial \Omega\right)$ the inequality (7.10) may be easily obtained by our taking possibly smaller constant $C\left(x_{0}\right)$.

\subsection{Zeros of the Jacobian.}

Theorem 7.2. For any quasiregular $f: \Omega \rightarrow \boldsymbol{R}^{n}, f \not \equiv$ constant in $a$ domain $\Omega$ let $N_{f}=\left\{x \in \Omega, J_{f}(x)=0\right\}$. Then mes $N_{f}=0$.

Proof. Assume that mes $N_{f}>0$ and that $x_{0}=0$ is a density point of $N_{f}$ i.e.,

$$
\lim _{r \rightarrow 0} \frac{\left|B(0, r)-N_{f}\right|}{|B(0, r)|}=0, \quad B(0, r) \subset \Omega
$$

The Hölder inequality gives

$$
\int_{B(0, r)}|D f(x)|^{n / 2} d x=\int_{B(0, r)-N_{f}}|D f(x)|^{n^{\prime 2}} d x \leqq\left|B(0, r)-N_{f}\right|^{1 / 2}\left(\int_{B(0, r)}|D f(x)|^{n} d x\right)^{1 / 2}
$$

Hence by the weak inverse Hölder inequality (3.7)

$$
\begin{gathered}
\left(\int_{B(0, r / 2)}|D f|^{n}\right)^{1 / n} \leqq 4 K C(n)\left(f_{B(0, r)}^{f}|D f|^{n / 2}\right)^{2 / n} \\
\leqq C(n, K)\left(\frac{\left|B(0, r)-N_{f}\right|}{|B(0, r)|}\right)^{1 / n}\left(f_{B(0, r)}|D f|^{n}\right)^{1 / n}=\varepsilon(r)\left(f_{B(0, r)}\left|D f^{\prime}\right|^{n}\right)^{1 / n},
\end{gathered}
$$

where in view of (7.11) $\varepsilon(r) \rightarrow 0$ as $r \rightarrow 0$. Iterating we get

$$
\left(\underset{B\left(0,2^{-k} r\right)}{f}|D f|^{n}\right)^{1 / n} \leqq \varepsilon(r) \varepsilon\left(\frac{r}{2}\right) \varepsilon\left(\frac{r}{4}\right) \ldots \varepsilon\left(\frac{r}{2^{k-1}}\right)\left(\underset{B(0, r)}{f}|D f|^{n}\right)^{1 / n}
$$


For any natural $N$ we choose $r_{0}=r_{0}(N)$ such that the numbers $\varepsilon\left(r_{0}\right), \varepsilon\left(r_{0} / 2\right)$, $\varepsilon\left(r_{0} / 4\right), \ldots, \varepsilon\left(r_{0} / 2^{k-1}\right), \ldots$ do not exceed $2^{-N}$ for $k=1,2, \ldots$ We then have

$$
\left(f_{B(0,2-k}|D f|^{n}\right)^{1 / n} \leqq\left(\frac{1}{2}\right)^{N k}\left(f_{B\left(0, r_{0}\right)}|D f|^{n}\right)^{1 / n}=r_{0}^{-N}\left(2^{-k} r_{0}\right)^{N}\left(f_{B\left(0, r_{0}\right)}|D f|^{n}\right)^{1 / n}
$$

For each $r \leqq r_{0}$ there exists $k$ such that $2^{-k} r_{0}<r \leqq 2^{-k+1} r_{0}$. Then

This means that

$$
\begin{gathered}
\left(\underset{B(0, r)}{f}|D f|^{n}\right)^{1 / n} \leqq 2\left({ }_{B\left(0,2^{-k+1} r_{0}\right)}|D f|^{n}\right)^{1 / n} \\
\leqq 2 r_{0}^{-N}\left(2^{-k+1} r_{0}\right)^{N}\left(f_{B\left(0, r_{0}\right)}|D f|^{n}\right)^{1 / n} \leqq 2^{1+N} r_{0}^{-N} r^{N}\left(\underset{B\left(0, r_{0}\right)}{f}|D f|^{n}\right)^{1 / n} .
\end{gathered}
$$

$$
\left(\int_{B(0, r)}|D f|^{n}\right)^{1 / n} \leqq C(N) r^{N+1} \quad \text { for } \quad r \leqq r_{0}=r_{0}(N) .
$$

By Theorem 5.2 (see also 5.5) we have

$$
\sup _{|x|<r / 2}|f(x)| \leqq C(n, K)\left(\int_{B(0, r)}|D f|^{n}\right)^{1 / n} \leqq C(n, K, N) r^{N+1}
$$

for any $N$. This, however, is incompatible with Corollary 7.2.

7.3. Outer Hausdorff measure. Our nearest aim is to show relations between capacity and the outer Hausdorff measure $\gamma_{\alpha}$. We return to the considerations of Paragraph 4.1.

The following Lemma generalizes the local Sobolev imbedding inequality.

Lemma 7.1. Let $Q_{0}$ be a cube in $\boldsymbol{R}^{n}$ and $u \in C_{0}^{1}\left(Q_{0}\right)$ and let $1<p \leqq \infty$, $0 \leqq n-\alpha<p$. Then there exists a constant $C=C(n, \alpha, p)$ such that

$$
|u(x)| \leqq C(n, \alpha, p)\left(\operatorname{diam} Q_{0}\right)^{1-n / p+\alpha / p} M_{\alpha, p} \nabla u(x)
$$

for each $x \in Q_{0}$. It reduces to Sobolev's inequality for $\alpha=0$.

Proof. We assume that $Q_{0}=Q(0, R)$ and $u$ is a function defined in $\boldsymbol{R}^{n}$ with zero values outside $Q_{0}$. For every $x \in \boldsymbol{R}^{n}$ we have

$$
u(x)=-\int_{0}^{\infty}\left(\frac{d}{d t} f_{Q(0,1)} u(x+t y) d y\right) d t=-\int_{0}^{\infty} f_{Q(0,1)}\langle y, \nabla u(x+t y)\rangle d y d t
$$


Hence

$$
\begin{aligned}
|u(x)| & \leqq \int_{0}^{\infty} f_{Q(0,1)}|\nabla u(x+t y)| d y d t=\int_{0}^{\infty}\left(f_{Q(x, r)}|\nabla u(y)| d y\right) d r \\
& \leqq \int_{0}^{R}\left(f_{Q(x, r)}|\nabla u|^{p}\right)^{1 / p} d r+\int_{R}^{\infty}\left(f_{Q(x, r)}|\nabla u|\right) d r \\
& \leqq 2^{(\alpha-n) / p} \int_{0}^{R}\left(\frac{1}{|Q(x, r)|^{\alpha / n}} \int_{Q(x, r)}|\nabla u|^{p}\right)^{1 / p} r^{(\alpha-n) / p} d r \\
& +\frac{1}{2^{n}(n-1) R^{n-1}} \int_{Q(0, R)}|\nabla u| \leqq 2^{(\alpha-n) / p} M_{\alpha, p} \nabla u(x) \int_{0}^{R} r^{(\alpha-n) / p} d r \\
& +\frac{(2 R)^{1-n / p+\alpha / p}}{2(n-1)}\left(\frac{1}{|Q(0, R)|^{\alpha / n}} \int_{Q(0, R)}|\nabla u|^{p}\right)^{1 / p} \\
& \leqq \frac{p 2^{(\alpha-n) / p}}{\alpha-n+p} R^{1-n / p+\alpha / p} M_{\alpha, p} \nabla u(x)+\frac{2^{(\alpha-n) / p}}{n-1} R^{1-n / p+\alpha / p} M_{\alpha, p} \nabla u(x) \\
& =\frac{(\alpha-n+n p) 2^{(\alpha-n) / p} R^{1-n / p+\alpha / p}}{(p-n+\alpha)(n-1)} \frac{M_{\alpha, p} \nabla u(x),}{(n-1)}
\end{aligned}
$$

which is (7.12).

Combining this with Lemma 4.1 we immediately get

Lemma 7.2. Under the hypotheses of Lemma 7.1 the inequality

$$
\gamma_{\alpha}\left\{x \in Q_{0} ;|u(x)|>\lambda\right\} \leqq \frac{5^{\alpha} C^{p}(n, \alpha, p)\left(\operatorname{diam} Q_{0}\right)^{p+\alpha-n}}{\lambda^{p}} \int_{Q_{0}}|\nabla u|^{p}
$$

holds for every $\lambda>0$.

Proof. By (7.12) and (4.4) we have

$$
\begin{aligned}
\gamma_{\alpha}\left\{x \in Q_{0} ;|u(x)|\right. & >\lambda\} \leqq \gamma_{\alpha}\left\{x \in Q_{0} ; M_{\alpha, p} \nabla u(x)>\lambda\left(\operatorname{diam} Q_{0}\right)^{n / p-\alpha / p-1} C^{-1}\right\} \\
& \leqq \frac{5^{\alpha}}{\left(\lambda C^{-1}\left(\operatorname{diam} Q_{0}\right)^{n / p-\alpha^{\prime} p-1}\right)^{p}} \int_{Q_{0}}|\nabla u|^{p} .
\end{aligned}
$$

As an important consequence of the estimation (7.13) we obtain

Lemma 7.3. Let $F$ be a compact subset of $\Omega \subset \boldsymbol{R}^{n}$ such that $\operatorname{Cap}_{n}(F, \Omega)=0$. Then $\gamma_{\alpha}(F)=0$ for every $0<\alpha \leqq n$. In particular, $\gamma_{1}(F)=0$ and thus $F$ does not contain any line-segment.

Proof. Let $u$ be an arbitrary $C_{0}^{1}(\Omega)$ function such that $u(x) \equiv 1$ on $F$ and let $Q_{0}$ be a cube containing $\Omega$. Therefore

$$
\gamma_{\alpha}(F) \leqq \gamma_{\alpha}\left\{x \in Q_{0} ;|u(x)| \geqq 1 / 2\right\} \leqq\left. C(n, \alpha) \int_{Q_{0}} \nabla u\right|^{n}=C(n, \alpha) \int_{\Omega}|\nabla u|^{n} .
$$


Since $u$ is an arbitrary $C_{0}^{1}(\Omega)$ function, relevant for the definition of the $n$-capacity of the pair $(F, \Omega)$, then $\gamma_{\alpha}(F) \leqq C(n, \alpha) \operatorname{Cap}_{n}(F, \Omega)=0$.

Finally using Corollary 7.1 we conclude:

Corollary 7.3. Let $f: \Omega \rightarrow \boldsymbol{R}^{n}$ be a quasiregular mapping such that $f \not \equiv$ const. Then for any $y$ the outer Hausdorff measure $\gamma_{\alpha}$ of the closed set $f^{-1}(y)$ is zero for any positive $\alpha$

$$
\gamma_{\alpha}\left(f^{-1}(y)\right)=0 .
$$

In particular, $f^{-1}(y)$ does not contain any line-segment.

7.4. An application to smooth $Q R$ mappings. We shall now show that any nonconstant and smooth quasiregular mapping $f: \Omega \rightarrow \boldsymbol{R}^{n}, n \geqq 3$ is a local homeomorphism. This is a basic fact distinguishing the two-dimensional theory from the general case $n \geqq 3$. For $n=2$ holomorphic functions supply examples of smooth quasiregular mappings having branch points e.g. $f(z)=z^{k}, k=2,3, \ldots, z=x^{1}+i x^{2}$.

The result proved below is weaker than the facts known in the literature. However, we include this weaker result here to illustrate the application of our methods.

We begin with the following simpler case:

Lemma 7.4. Let $f: \boldsymbol{R}^{n} \rightarrow \boldsymbol{R}^{n}, n \geqq 3$ be a quasiregular mapping whose components are homogeneous polynomials of degree $k, k \geqq 1$. Then $f$ is a homeomorphism of $\boldsymbol{R}^{n}$ onto itself.

Proof. First of all we observe that the Jacobian $J_{f}(x)$ of the map $f$ does not vanish in $\boldsymbol{R}^{n}-\{0\}$. Contradicting this fact suppose that $J_{f}\left(x_{0}\right)=0$ for some $x_{0} \neq 0$. Since $f$ is quasiregular, $D f\left(x_{0}\right)=0$. On the other hand, $D f(x)$ is homogeneous of order $k-1$; thus $D f$ vanishes on the line $\left\{t x_{0} ; t \in \boldsymbol{R}^{1}\right\}$. As a consequence of that we get $f\left(t x_{0}\right) \equiv$ const $=0$ for all real $t$. This contradicts Corollary 7.3. In a similar way we prove that $f(x) \neq 0$ for $x \neq 0$. Therefore $f$ is a local diffeomorphism of $\boldsymbol{R}^{n}-\{0\}$ into itself.

Let us now consider the map $\varphi: S^{n-1} \rightarrow S^{n-1}$ defined on the unit sphere $S^{n-1}$ by the formula

$$
\varphi(x)=\frac{f(x)}{|f(x)|}
$$

This mapping is a local homeomorphism (even local diffeomorphism) follows from the following observation: $\varphi=f \circ h$, where the map $h: S^{n-1} \rightarrow \boldsymbol{R}^{n}-\{0\}$ has the form $h(x)=\lambda(x) x$. Here $\lambda>0$ is the scalar smooth function, $\lambda(x)=$ $|f(x)|^{-1 / k}$. Such mappings as $h$ transform diffeomorphically the unit sphere onto a closed smooth $(n-1)$ surface. Since $f$ is a local diffeomorphism in $\boldsymbol{R}^{\boldsymbol{n}}-\{0\}$, then $\varphi$ is a local diffeomorphism on the unit sphere.

The crucial point in our proof is the following topological theorem: Any local homeomorphism of $S^{n-1}$ into itself must be a homeomorphism onto $S^{n-1}$. 
Here the assumption $n \geqq 3$ is essential. On the basis of this theorem we see that $\varphi$ is a diffeomorphism of $S^{n-1}$ onto $S^{n-1}$. We shall derive from this that $f: \boldsymbol{R}^{n}-\{0\} \rightarrow \boldsymbol{R}^{n}-\{0\}$ is a diffeomorphism. Since we know that $f$ is a local diffeomorphism, it suffices to prove that $f$ is one to one. Let $f\left(x^{1}\right)=f\left(x^{2}\right)$; then $\varphi\left(x^{1} /\left|x^{1}\right|\right)=\varphi\left(x^{2} /\left|x^{2}\right|\right)$, so $x^{1} /\left|x^{1}\right|=x^{2} /\left|x^{2}\right|$. The homogeneity condition implies $f\left(x^{1}\right) /\left|x^{1}\right|^{k}=f\left(x^{2}\right) /\left|x^{2}\right|^{k}$, whence $\left|x^{1}\right|=\left|x^{2}\right|$ and $x^{1}=x^{2}$. The lemma is proved.

Let us remark that the inverse mapping $f^{-1}: \boldsymbol{R}^{n} \rightarrow \boldsymbol{R}^{n}$ is Hölder continuous with the Hölder exponent $\alpha=1 / k$. The following theorem generalizes Lemma 7.4.

Theorem 7.3. Let $f: \Omega \rightarrow \boldsymbol{R}^{n}$ be a non-constant quasiregular mapping of the class $C^{\infty}(\Omega)$, where $\Omega$ is a domain in $\boldsymbol{R}^{n}, n \geqq 3$. Then $f$ is a local homeomorphism.

Proof. We investigate $f$ in a sufficiently small neighbourhood of an arbitrary point $x_{0} \in \Omega$. For simplicity we assume that $x_{0}=0 \in \Omega$ and $f(0)=0$. By Corollary 7.2 there exist an integer $N$ and a constant $C>0$ such that

$$
\sup _{|x| \leqq r}|f(x)| \geqq C r^{N}
$$

for sufficiently small $r>0$. We use the Taylor expansion formula

$$
f(x)=P_{1}(x)+\ldots+P_{N}(x)+O\left(|x|^{N+1}\right)
$$

where $P_{k}$ are homogeneous polynomials of degree $k$. In view of (7.15) one of them does not vanish identically. Therefore we can write

$$
f(x)=h(x)+R(x)
$$

where $h$ is a homogeneous polynomial mapping of degree, say $k, 1 \leqq k \leqq N$ and $R$ is a smooth mapping such that $|R(x)|=O\left(|x|^{k+1}\right)$. Since $f$ is $K$-quasiregular, i.e., $|D f(x)|^{n} \leqq K J_{f}(x)$, then $|D h(x)|^{n} \leqq K J_{h}(x)+O\left(\mid x^{k n+1-n}\right)$ and by homogeneity of $h$ we infer that $h$ is also a $K$-quasiregular mapping. Now Lemma 7.4 implies that $h$ is a homeomorphic map of $\boldsymbol{R}^{n}$ onto itself. This makes i possible to examine the map $f\left(h^{-1}(y)\right)=y+R\left(h^{-1}(y)\right)$ defined in a neighbourhood of $y=0$.

Obviously $R\left(h^{-1}(y)\right)=O\left(\left|h^{-1}(y)\right|^{k+1}\right)=O\left(|y|^{(k+1) / n}\right)$ and $R h^{-1}$ is a $C^{\infty}$ map for $y \neq 0$. This shows that $R h^{-1}$ is a $C^{1}$ mapping such that $D\left(R h^{-1}\right)(0)=0$. Finally we conclude that $f h^{-1}$ is a $C^{1}$ diffeomorphism in a neighbourhood of $y=0$ (since $D f h^{-1}(0)=I$-the identity matrix) and consequently $f$ is a homeomorphism in a neighbourhood of $x=0$. 


\section{MEASURABILITY AND INTEGRATION}

We will now briefly discuss the behaviour of the Lebesgue measure under quasiconformal and quasiregular mappings and the closely related change of variables formula for definite integrals.

8.1. Lusin's condition $\mathscr{N}$. Let $f: \Omega \rightarrow \boldsymbol{R}^{n}$ ( $\Omega$ is a domain in $\boldsymbol{R}^{n}$ ) be a mapping. Then $f$ is said to satisfy the condition $\mathscr{N}$ if for every set $E \subset \Omega$ of measure zero the image $f(E)$ is also a set of measure zero. Let us recall that if $f$ is a continuous mapping, the condition $\mathscr{N}$ is necessary and sufficient in order that the mapping transforms every measurable set into a measurable set. Indeed, let $f$ fulfil the condition $\mathcal{N}$. Since a measurable set $A \subset \Omega$ is a sum of a set $E$ of measure zero and an ascending sequence of compact sets $E_{i}$, then $f(A)=\cup_{i} f\left(E_{i}\right) \cup f(E)$, where $f\left(E_{i}\right)$ are compact and $|f(E)|=0$, whence $f(A)$ is measurable. Conversely, assuming that $f$ transforms measurable sets into measurable sets, let us suppose that there exists a set $E$ of measure zero such that $F=f(E)$ has positive measure. Then one can find a subset $F^{\prime} \subset F$ which is not measurable. Let $E^{\prime} \subset E$ be the inverse image of $F^{\prime}$ under the map $f: E \rightarrow F$. Then $E^{\prime}$ is measurable as a subset of the zero measure set $E$ but the image $f\left(E^{\prime}\right)=F^{\prime}$ is not measurable, contrary to the assumption. In connection with the condition $\mathscr{N}$ we recall another essentially more restrictive condition, introduced by S. Banach [33] and called the condition $S$.

We say that the mapping $f: \Omega \rightarrow \boldsymbol{R}^{n}$ satisfies the condition $S$ if for each number $\varepsilon>0$ there exists a $\sigma>0$ such that, for each measurable set $E \subset \Omega$, the inequality $|E|<\sigma$ implies that $f(E)$ is measurable and $|f(E)| \leqq \varepsilon$.

Obviously every mapping which fulfils the condition $S$ also fulfils the condition $\mathscr{N}$.

Lemma 8.1. Let $f: \Omega \rightarrow \boldsymbol{R}^{n}$ be a mapping of the Sobolev class $W_{p}^{1}(\Omega), p>n$. Then $f$ satisfies the condition $S$. Moreover, for each measurable subset $E \subset \subset \Omega$ we have

$$
|f(E)| \leqq C(n, p)|E|^{1-n / p}\left(\int_{E}|D f(x)|^{p} d x\right)^{n / p},
$$

where $C(n, p)$ depends only on $n$ and $p$.

Proof. First we examine a set $E \subset \subset \Omega$ which is the union of disjoint cubes $Q_{j} \subset \subset \Omega, j=1,2, \ldots, E=\cup_{j} Q_{j}$. For every cube $Q_{j}$ we have on account of (1.28)

$$
\left|f\left(Q_{j}\right)\right| \leqq 2^{n}\left(\operatorname{diam} f\left(Q_{j}\right)\right)^{n} \leqq C(n, p)\left(\operatorname{diam} Q_{j}\right)^{(1-(n / p)) n}\left(\int_{Q_{j}}|D f|^{p}\right)^{n / p} .
$$

Hence by the Hölder inequality we obtain

$$
\begin{gathered}
\sum_{j}\left|f\left(Q_{j}\right)\right| \leqq C(n, p)\left(\sum_{j}\left(\operatorname{diam} Q_{j}\right)^{n}\right)^{1-n / p}\left(\sum_{j} \int_{Q_{j}}|D f|^{p}\right)^{n / p} \\
\leqq C(n, p)\left(\sum_{j}\left|Q_{j}\right|\right)^{1-n / p}\left(\int_{\cup Q_{j}}|D f|^{p}\right)^{n / p}=C(n, p)|E|^{1-n / p}\left(\int_{E}|D f|^{p}\right)^{n / p} .
\end{gathered}
$$


Here $\left|f\left(Q_{j}\right)\right|$ means the outer Lebesgue measure of $f\left(Q_{j}\right)$. Now let $E$ be an arbitrary measurable subset, $E \subset \subset \Omega$, and let $Q_{j} \subset \subset \Omega$ be cubes with disjoint interiors such that $E \subset \cup_{j} Q_{j}, \Sigma_{j}\left|Q_{j}\right| \leqq|E|+\varepsilon$ for some $\varepsilon>0$. Then by the inequality (8.2)

$$
\begin{gathered}
|f(E)| \leqq \Sigma_{j}\left|f\left(Q_{j}\right)\right| \leqq C(n, p)(|E|+\varepsilon)^{1-n / p}\left(\int_{\cup Q_{j}}|D f|^{p}\right)^{n / p} \\
\leqq C(n, p)(|E|+\varepsilon)^{1-n / p}\left(\int_{Q}|D f|^{p}\right)^{n / p} .
\end{gathered}
$$

In particular, if $|E|=0$, we immediately get $|f(E)|=0$ since $\varepsilon$ may be chosen arbitrarily small. In other words, $f$ fulfils the condition $\mathscr{N}$. Hence $f(E)$ is measurable whenever $E$ is measurable. Now one can easily derive the inequality (8.1) from the last estimation and afterwords deduce the condition $S$. An explicit calculation is omitted, being standard.

Lemma 8.1 implies at once

Theorem 8.1. Any quasiregular mapping fulfils the condition $\mathscr{N}$.

8.2. Condition $\mathscr{N}^{-1}$. The map $f: \Omega \rightarrow \boldsymbol{R}^{n}$ is said to satisfy the condition $\mathscr{N}^{-1}$ if for each set $F \subset \boldsymbol{R}^{n}$ of measure zero the preimage $f^{-1}(F)$ is also a set of measure zero.

Theorem 8.1. Any non-constant quasiregular map $f: \Omega \rightarrow \boldsymbol{R}^{n}$ satisfies condition $\mathscr{N}^{-1}$.

Proof. We have to show that $|f(E)|=0$ implies $|E|=0$. Assume the contrary: for some $E$ of positive measure $|f(E)|=0$. Since $f$ is differentiable almost everywhere (Theorem 5.3) and the Jacobian $J_{f}(x)>0$ for almost each $x \in E$ (Theorem 7.2), there is a point $x_{0} \in E$ which satisfies the following conditions:

a) $x_{0}$ is a density point of $E$,

b) $f$ is differentiable at $x_{0}$,

c) $J_{f}\left(x_{0}\right)>0$,

d) $x_{0}$ is a Lebesgue point of the function $|D f|^{p}$.

Let $Q_{j}=Q_{j}\left(x_{0}\right), j=1,2, \ldots$ be a sequence of cubes shrinking into $x_{0}$. We then have

$$
\begin{gathered}
\frac{\left|f\left(Q_{j}\right)\right|}{\left|Q_{j}\right|}=\frac{\left|f\left(Q_{j}-E\right)\right|}{\left|Q_{j}\right|} \leqq C(n, p)\left(\frac{\left|Q_{j}-E\right|}{\left|Q_{j}\right|}\right)^{1-n / p}\left(\frac{1}{\left|Q_{j}\right|} \int_{Q_{j}-E}|D f|^{p}\right)^{n / p} \\
\leqq C(n, p)\left(\frac{\left|Q_{j}-E\right|}{\left|Q_{j}\right|}\right)^{1-n / p}\left(\int_{Q_{j}}|D f|^{p}\right)^{n / p}
\end{gathered}
$$

in view of (8.1). Letting $j \rightarrow \infty$, we get by b) that the left-hand side of the above estimation converges to $J_{f}\left(x_{0}\right)>0$. Now a) and d) imply that the right-hand side converges to 0 . The obtained contradiction proves the theorem. 
8.3. Banach indicatrix. First version of change of variables formula. A suitable instrument for studying quasiregular mappings is the transformation formula for definite integrals under general transformations not necessary one to one. For this purpose we introduce the so-called Banach indicatrix $N(y ; f, \Omega)$ of a map $f: \Omega \rightarrow \boldsymbol{R}^{n}$ (other names used in literature are crude multiplicity function or the counting function).

Definition 8.1. Let $f: \Omega \rightarrow \boldsymbol{R}^{n}$ be a mapping and lot $E$ be a subset of $\Omega$. Then

$$
N(y ; f, E)=\operatorname{card}\{x \in E ; f(x)=y\}
$$

for each $y \in \boldsymbol{R}^{n}$. We admit the values $+\infty$ for the function $N(y ; f, E)$.

Theorem 8.3. Let $f: \Omega \rightarrow \boldsymbol{R}^{n}$ be a continuous mapping satisfying the condition $\mathscr{N}$. Assume that $f$ is differentiable almost everywhere and the Jacobian $J_{f}(x)$ is integrable on $\Omega$. Then the function $N(y ; f, \Omega)$ is integrable in $\boldsymbol{R}^{n}$ ard

$$
\int_{\Omega}\left|J_{f}(x)\right| d x=\int_{\mathbf{R}^{n}} N(y ; f, \Omega) d y .
$$

Notice that in view of Lemmas 1.7, 1.8 and Proposition 5.1 any map of the class $W_{p}^{1}(\Omega)$ with $p>n$ satisfies all the hypotheses of this thcorem. In particular, quasiregular mappings are in the range of our considerations. The proof of Theorem 8.3 is based on an auxiliary Lemma 8.2.

By $\mathscr{M}_{k}, k=0,1,2, \ldots$ we denote the division of $R^{n}$ into half-closed cubes of the side-length equal to $2^{-k}$, no two of which have points in common. Any cube from $\mathscr{M}_{k+1}$ arises by the dyadic division of a cube from $\mathscr{M}_{k}$. By $\mathscr{M}$ we denote the union of the families $\mathscr{M}_{k}, k=0,1,2, \ldots, \mathscr{M}=\bigcup_{k} \mathscr{M}_{k}$.

Lemma 8.2. Let $f$ satisfy the hypotheses of Theorem 8.3. Then for every $m=1,2, \ldots$ there exists a subfamily $\mathscr{F}_{m} \subset \cup_{k=1}^{\infty} \mathscr{M}_{k}$ of disjoint cubes contained in $\Omega$ such that

a) ||$f(I)\left|-\int_{I}\right| J_{f}(x)|d x| \leqq \frac{1}{m}|I|$ for each $I \in \mathscr{F}_{m}$,

b) $\left|\Omega-\bigcup_{I \in \mathscr{F}_{m}} I\right|=0$,

c) each cube $I \in \mathscr{F}_{m+1}$ is a subcube of a cube belonging to the family $\mathscr{F}_{m}$.

Proof. Fix the number $m$. We begin the construction of the family $\mathscr{F}_{m} \subset \bigcup_{k} \geqq_{m} \mathscr{M}_{k}$ with the choice of cubes from $\mathscr{M}_{m}$ which are contained in $\Omega$ and satisfy the condition a). Suppose by induction that the cubes from $\mathscr{M}_{k}$ are chosen as cubcs belonging to $\mathscr{F}_{m}, k \geqq m$. Now each cube $I \in \mathscr{M}_{k+1}$ which is contained in $\Omega$, has no points in common with any cube chosen before and fulfils the condition a) will be included to $\mathscr{F}_{m}$. 
The condition c) immediately follows from the above construction of $\mathscr{F}_{m}$ and from the observation that every two cubes from $\mathscr{M}$ are either disjoint or one includes the other. To verify the condition b) we suppose, by contradiction, that $\Omega-\bigcup_{I \in \mathscr{F}_{m}} I$ is a set of positive measure. We find $x_{0} \in \Omega-\bigcup_{I \in \mathscr{F}_{m}} I$, which will be a differentiability point of $f$ and a Lebesgue point of the function $\left|J_{f}\right| \in L^{1}(\Omega)$. There exists $k_{0}=k_{0}$ (dist $\left.\left(x_{0}, \partial \Omega\right), m\right) \geqq m$ such that for every $k \geqq k_{0}$ the unique cube $I_{k} \in \mathscr{M}_{k}$ such that $x_{0} \in I_{k}$ is contained in $\Omega$. Since $x_{0} \notin \bigcup_{I \in \mathscr{F}_{m}} I$, then $I_{k}$ cannot be a subcube of a cube belonging to the family $\mathscr{F}_{m}$, and, in view of the properties of $\mathscr{M}, I_{k}$ is disjoint with every cube from $\mathscr{F}_{m} \cap \bigcup_{m \geqq_{s} \geqq_{k}} \mathscr{M}_{s}$. This means that

$$
\left|\frac{\left|f\left(I_{k}\right)\right|}{\left|I_{k}\right|}-f_{I_{k}}\right| J_{f}(x)|d x|>\frac{1}{m} \text { for } k \geqq k_{0} .
$$

On the other hand, since $I_{k}$ are shrinking into $x_{0}$,

$$
\lim _{k \rightarrow \infty} f_{I_{k}}\left|J_{f}(x)\right| d x=\left|J_{f}\left(x_{0}\right)\right| \text { and also } \lim _{k \rightarrow \infty} \frac{\left|f\left(I_{k}\right)\right|}{\left|I_{k}\right|}=\left|J_{f}\left(x_{0}\right)\right| .
$$

The last statement follows from the differentiability of $f$ at the point $x_{0}$, i.e., $f(x)-f\left(x_{0}\right)=D f\left(x_{0}\right)\left(x-x_{0}\right)+O\left(\left|x-x_{0}\right|\right)$. This gives the contradiction.

The proof of Theorem 8.3. We consider a sequence of integer valued functions $g_{m}: \boldsymbol{R}^{n} \rightarrow \boldsymbol{N}=\{0,1, \ldots, \infty\}$ defined by

$$
g_{m}(y)=\sum_{I \in \mathscr{F}_{m}} \chi_{f(I)}(y),
$$

where $\chi_{F}$ stands for the characteristic function of a measurable subset $F \subset \boldsymbol{R}^{\boldsymbol{n}}$. For every $m$ we have the estimation

$$
-\frac{1}{m}|\Omega|+\int_{\Omega}\left|J_{f}(x)\right| d x \leqq \int_{R^{n}} g_{m}(y) d y \leqq \int_{\Omega}\left|J_{f}(x)\right| d x+\frac{1}{m}|\Omega| .
$$

In fact, if $I \in \mathscr{F}_{m}$, then by the condition a) of Lemma 8.2. we have

$$
-\frac{1}{m}|I|+\int_{I}\left|J_{f}(x)\right| d x \leqq \int_{R^{n}} \chi_{f(I)}(y) d y \leqq \int_{I}\left|J_{f}(x)\right| d x+\frac{1}{m}|I| .
$$

Summing up over all the cubes from $\mathscr{F}_{m}$ and taking into account the condition b) we get (8.4), noting that $g_{m}$ are integrable. Furthermore, we assert that

$$
0 \leqq g_{1}(y) \leqq g_{2}(y) \leqq \ldots \leqq N(y ; f, \Omega) \text { and } \lim _{m \rightarrow \infty} g_{m}(y)=N(y ; f, \Omega)
$$

for every $y \in \boldsymbol{R}^{n}-f(E)$, where $E=\bigcup_{m \geqq 1}\left(\Omega-\bigcup_{I \in \mathscr{F}_{m}} I\right)$. The above sequence of inequalities follows from the property $c)$ : To prove (8.5) we observe that for $y \in \boldsymbol{R}^{n}-f(E), f^{-1}(y) \subset \bigcup_{I \in \mathscr{F}_{m}} I, m=1,2, \ldots$ Consider the case $1 \leqq \operatorname{card} f^{-1}(y)=$ $N=N(y ; f, \Omega)<\infty$. Let $x_{1}, x_{2}, \ldots, x_{N} \in \Omega$ be district points such that $y=f\left(x_{j}\right)$, $j=1, \ldots, N$. Therefore $x_{j} \in \bigcup_{I \in \mathscr{F}_{m}} I$ for $m=1,2, \ldots, j=1,2, \ldots, N$. Since the side-length of any cube $I \in \mathscr{F}_{m} \subset \bigcup_{k} \geqq m \mathscr{M}_{k}$ does not exceed $2^{-m}$, then for sufficiently 
large $m$ the points $x_{1}, x_{2}, \ldots, x_{N}$ belong to the different cubes of the family $\mathscr{F}_{m}$. The number of such cubes is obviously equal to $g_{m}(y)$, which means $\lim _{m \rightarrow \infty} g_{m}(y)=$ $N(y ; f, \Omega)$. The case when card $\left(f^{-1}(y)\right)=\infty$ is treated similarly and the case when $f^{-1}(y)=\emptyset$ is obvious. Finally we observe that $E$ is the set of measure zero because of the property b). Since $f$ is assumed to fulfil the condition $\mathcal{N}$, then $f(E)$ has measure zero also. By (8.4), (8.5) and by Lebesgue's theorem on integration of monotone sequences we conclude that $N(y ; f, \Omega)$ is integrable in $\boldsymbol{R}^{n}$ and the formula (8.3) holds. This completes the proof of Theorem 8.3.

The integral formula (8.3) can also be used to derive the condition $\mathscr{N}^{-1}$ for quasiregular mappings. In fact, this method gives a slightly more general result, which we state here as follows.

Le m ma 8.3. Let $f$ satisfy all the hypotheses of Theorem 8.3. Assume additionally that the Jacobian $J_{f}(x)$ is positive for almost all $x \in \Omega$. Then $f$ fulfils the condition $\mathscr{N}^{-1}$.

Proof. Let $E$ be an arbitrary measurable subset of $\Omega$ such that $f(E)=0$. For every open set $U \supset f(E)$ the preimage $f^{-1}(U)$ is open and by (8.3) we have

$$
\begin{aligned}
\int_{E}\left|J_{f}(x)\right| d x \leqq \int_{f^{-1}(U)}\left|J_{f}(x)\right| d x & =\int_{R^{n}} N\left(y ; f, f^{-1}(U)\right) d y=\int_{U} N\left(y ; f, f^{-1}(U)\right) d y \\
& \leqq \int_{U} N(y ; f, \Omega) d y .
\end{aligned}
$$

Since $N(y ; f, \Omega)$ is integrable on $\boldsymbol{R}^{n}$ and $U$ is an arbitrary open set covering the set $f(E)$ of measure zero, we get $\int_{E}\left|J_{f}(x)\right| d x=0$.

Hence we conclude that $|E|=0$ on account of the assumption $\left|J_{f}(x)\right|>0$ for a.e. $x \in \Omega$. Therefore the lemma is proved.

Theorem 8.4. Let $f: \Omega \rightarrow \boldsymbol{R}^{n}$ be a continuous mapping of class $W_{n}^{1}(\Omega)$, differentiable almost everywhere, $J_{f}(x)>0$ a.e. and additionally satisfying the condition $\mathcal{N}$. Then for every $u \in L^{\infty}(\Omega)$

$$
\int_{\Omega} u(f(x)) J_{f}(x) d x=\int_{R^{n}} u(y) N(y ; f, \Omega) d y .
$$

Proof. Let us first examine the case when $u=\chi_{V}$-the characteristic function of an open set $V \subset \boldsymbol{R}^{n}$. Then $f^{-1}(V)$ is open and by (8.3) we can write

$$
\begin{gathered}
\int_{\Omega} \chi_{V}(f(x)) J_{f}(x) d x=\int_{f^{-1}(V)} J_{f}(x) d x=\int_{V} N\left(y ; f, f^{-1}(V)\right) d y=\int_{V} N(y ; f, \Omega) d y \\
=\int_{R^{n}} \chi_{V}(y) N(y ; f, \Omega) d y .
\end{gathered}
$$

We used the obvious equality $N\left(y ; f, f^{-1}(V)\right)=N(y ; f, \Omega)$ for each $y \in V$. Since both sides of (8.6) are additive functionals with respect to $u$, we get (8.6) for any piecewise constant function $u(y)=\sum_{i=1}^{m} C_{i} \chi_{V_{i}}$, where $V_{i}$ are disjoint open sub- 
scts of $\boldsymbol{R}^{n}$. Since any $u \in L^{\infty}\left(\boldsymbol{R}^{n}\right)$ may be approximated by a sequence of piecewise constant functions $u_{k}$ boundedly convergent to $u$ almost everywhere, the sequence $u_{k}(f(x))$ converges boundedly to $u(f(x))$ almost everywhere in $\Omega$. Here we used the essential fact that $f$ satisfies the condition $\mathscr{N}^{-1}$; see Lemma 8.3. By the Lebesgue convergence theorem we obtain (8.6) in the form asserted in the theorem.

8.4. Total variation in the Banach sense. Let $f: \Omega \rightarrow \boldsymbol{R}^{n}$ be a quasiregular mapping. The set function $\varphi$ defined by the formula $\varphi(E)=|f(E)|$ is not additive in general. Therefore the theory of derivation of set functions is not applicable to $\varphi$. A relevant tool in the study of the measure of the image $s \in t s f(E)$ is the theory of variation.

We recall the notion of the total variation of a map $f: \Omega \rightarrow \boldsymbol{R}^{n}$. Assume that $f$ is continuous. Then on each open set $D \subset \Omega$ the Banach variation is defined by

$$
V_{B}(f, D)=\sup _{\mathscr{F}} \sum_{I \in \mathscr{F}}|f(I)|,
$$

where the supremum is taken over all finite systems $\mathscr{F}$ of closed intervals contained in $D$ no two of which have interior point in common. An interval $I$ in $\boldsymbol{R}^{n}$ is a point set determined by the inequalities $a^{i} \leqq x^{i} \leqq b^{i},\left(a^{i}<b^{i}\right) i=1,2, \ldots, n$. Let us observe that $V_{B}(f ; \cdot)$ considered as a set function defined on open subsets of $\Omega$ is completely additive in the sense that

$$
V_{B}\left(f, \cup D_{i}\right)=\Sigma_{i} V_{B}\left(f, D_{i}\right)
$$

for any sequence of open disjoint subsets $D_{i} \subset \Omega$.

From the inequality (8.1) we immediately see that any $f: \Omega \rightarrow \boldsymbol{R}^{n}$ of the class $W_{p}^{1}(\Omega), p>n$ is a mapping of bounded variation. More precisely,

$$
\begin{gathered}
\quad V_{B}(f ; \Omega)=\sup _{\mathscr{F}} \sum_{I \in \mathscr{F}}|f(I)| \leqq C(n, p) \sup _{\mathscr{F}} \sum_{I \in \mathscr{F}}|I|^{1-n / p}\left(\int_{I}|D f|^{p}\right)^{n / p} \\
\leqq C(n, p) \sup _{\mathscr{F}}\left(\sum_{I \in \mathscr{F}}|I|\right)^{1-n / p}\left(\int_{\cup I}|D f|^{p}\right)^{n / p} \leqq C(n, p)|\Omega|^{1-n / p}\left(\int_{\Omega}|D f|^{p}\right)^{n / p}<\infty .
\end{gathered}
$$

To simplify further considerations from now on we assume that $f \in W_{p}^{1}(\Omega)$ with some $p>n$. The formula (8.7) may be simply extended for $D$ being composed of closed intervals. In this way $V_{B}(f ; \cdot)$ may be viewed as a function of intervals. This function is additive in the sense that

$$
V_{B}\left(f ; I_{1} \cup I_{2}\right)=V_{B}\left(f ; I_{1}\right)+V_{B}\left(f ; I_{2}\right)
$$

whenever $I_{1}, I_{2}$ and $I_{1} \cup I_{2}$ are intervals contained in $\Omega$ and $I_{1}, I_{2}$ are nonoverlapping. In fact, the inequality $V_{B}\left(f ; I_{1}\right)+V_{B}\left(f ; I_{2}\right) \leqq V_{B}\left(f ; I_{1} \cup I_{2}\right)$ is obvious. To show the opposite inequality we consider an arbitrary finite family $\mathscr{F}$ of non-overlapping intervals $I \subset I_{1} \cup I_{2}$. Let $\mathscr{F}_{1}=\left\{I \cap I_{1} ; I \in \mathscr{F}\right\}$ and $\mathscr{F}_{2}=\left\{I \cap I_{2} ; I \in \mathscr{F}\right\}$. For each $I \in \mathscr{F}$ we have $|f(I)| \leqq\left|f\left(I \cap I_{1}\right)\right|+\left|f\left(I \cap I_{2}\right)\right|$. Hence

$$
\sum_{i \in \mathscr{F}}|f(I)| \leqq \sum_{I^{\prime} \in \mathscr{F}_{1}}\left|f\left(I^{\prime}\right)\right|+\sum_{I^{\prime \prime} \in \mathscr{F}_{2}}\left|f\left(I^{\prime \prime}\right)\right| \leqq V_{B}\left(f ; I_{1}\right)+V_{B}\left(f ; I_{2}\right)
$$


and

$$
V_{B}\left(f ; I_{1} \cup I_{2}\right) \leqq V_{B}\left(f ; I_{1}\right)+V_{B}\left(f ; I_{2}\right) .
$$

The inequality (8.9) shows that $V_{B}(f ; \cdot)$ is absolutely continuous, that is, there exists a number $\sigma>0$ corresponding to each $\varepsilon>0$ such that for every non-overlapping intervals $I_{1}, I_{2}, \ldots, I_{N} \subset \Omega$ the inequality $\left|\bigcup_{i} I_{i}\right|<\sigma$ implies $V_{B}\left(f ; \cup I_{i}\right)<\varepsilon$.

Now we recall the fundamental Lebesgue theorem on the derivation of additive functions of intervals.

Theorem 8.5. Let $\mu$ be an additive function of intervals of bounded variation and absolutely continuous. Then $\mu$ is almost everywhere derivable ard

$$
\mu(I)=\int_{I} \mu^{\prime}(x) d x
$$

for each interval $I \subset \Omega$ (see [33]).

The derivative of $\mu$ at a point $x \in \Omega$, denoted by $\mu^{\prime}(x)$, is defincd as

$$
\mu^{\prime}(x)=\lim _{k \rightarrow \infty} \frac{\mu\left(Q_{k}\right)}{\left|Q_{k}\right|},
$$

where the limit is taken over an arbitrary sequence $Q_{k}$ of cubes shrinking into $x$ such that $x \in Q_{k}, k=1,2, \ldots$. It can be shown that for each $f \in W_{p}^{1}(\Omega), p>n$ the derivative of total variation of $f$ is almost everywhere equal to the absolute value of the Jacobian of $f$. In this way we proved

Lemma 8.4. For any map $f \in W_{p}^{1}(\Omega), p>n$, the formula

$$
V_{B}(f ; U)=\int_{U}\left|J_{f}(x)\right| d x=\int_{R^{n}} N(y ; f, U) d y
$$

holds, where $U$ is an arbitrary open set in $\Omega$.

8.5. Topological index. Second version of change of variables formula. Let $f: \Omega \rightarrow \boldsymbol{R}^{n}$ be a quasiregular map. The topological index of a point $z$ in the target space $\boldsymbol{R}^{n}$ with respect to the map $f$ restricted to a sub-domain $D \subset \Omega$ is defined only for $(f, D)$ admissible points $z$. If $D$ is a subdomain of $\Omega$ with compact closure $\bar{D} \subset \Omega$, a point $z$ of the target space of $f$ is called $(f, D)$ admissible if $z \notin f(\partial D)$, where $\partial D$ is the boundary of $D, \partial D=\bar{D} \backslash D$. The set $C_{D} \equiv C(f, D)$ of $(f, D)$ admissible points is then the complement of the compact set $f(\partial D)$ and decomposes into an at most countable number of $N$ disjoint components, $1 \leqq N \leqq \infty, C_{D}=\cup_{i=1}^{N-1} C_{i} \cup C_{\infty}$, where by $C_{\infty}$ we denote the unique component containing the point $\infty$ of $\overline{\boldsymbol{R}}^{n}$. $C_{\infty}$ will be called the outer domain of the map $f$.

We shall use an analytic definition of the topological index [17]. For that we shall need a special family of auxiliary "bump" measures, compactly supported approximations of Dirac measures in $\boldsymbol{R}^{n}$.

Let $\varphi^{\varepsilon}(r), 0 \leqq \varepsilon \leqq 1$, be an arbitrary family of $C^{\infty}$ functions defined for $r \geqq 0$ and such that $\varphi^{\varepsilon}(r) \equiv 0$ for $r \geqq \varepsilon, \varphi^{\varepsilon}(r) \equiv$ const in a neighbourhood of $r=0, \varphi^{\varepsilon}(r) \geqq 0$ 
and

$$
\int_{R^{n}} \varphi^{\varepsilon}(|y|) d y=1 .
$$

Obviously the functions $\varphi^{\varepsilon}(r)$ can be taken in the form $\varphi^{\varepsilon}(r)=1 / \varepsilon^{n} \Psi(r / \varepsilon)$ with some fixed smooth $\Psi \in C_{0}^{\infty}(R), \quad \Psi(t) \geqq 0, \Psi(t)=0$ for $|t| \geqq 1, \int_{R^{n}} \Psi(|y|) d y=1$. With $\varphi^{\varepsilon}(r)$ fixed we set

$$
\varrho_{z}^{\varepsilon}(y)=\varphi^{\varepsilon}(|z-y|) d y
$$

and obtain a family of $n$-forms in $\boldsymbol{R}^{n}$ such that

a) $\varrho_{z}^{\varepsilon}$ is compactly supported and for each $(f, D)$ admissible $z$ and for $\varepsilon$ sufficiently small the support of $\varrho_{z}^{\varepsilon}$, is contained in the open component of $C_{D}$ containing the point $z$,

b) $\varrho_{z}^{\varepsilon}$ is normalized

$$
\int_{R^{n}} \varrho_{z}^{\varepsilon}(y) d y=1
$$

Definition 8.2. The topological index $\mu(z ; f, D)$ of the $(f, D)$ admissible point $z$ is defined by the formula

$$
\mu(z ; f, D)=\lim _{\varepsilon \rightarrow 0} \int_{D} f^{*} \varrho_{z}^{\varepsilon},
$$

whore $f^{*} \varrho_{z}^{\varepsilon}=\varphi^{\varepsilon}(|z-f(x)|) J_{f}(x) d x$ is the "pulled back" $n$-form on $D$.

Since $z \notin f(\partial D), f^{*} \varrho_{z}^{\varepsilon}$ is compactly supported in $D$ (for $\varepsilon<\operatorname{dist}(z, f(\partial D))$ ), and the integral in (8.12) has sense for $\varepsilon$ small enough. The topological index $\mu(z ; f, D)$ is defined by the integral formula (8.12) as a real number, non-negative for (orientation preserving) q.r. mappings, depending on the casual choice of the bump forms $\varrho_{z}^{\varepsilon}$. The basic property of the function $\mu(z ; f, D)$ is that it is integervalued and independent of the auxiliary choices. As a matter of fact it depends on the homotopy class of the restriction $f \mid \partial D$ of the map $f$ to the boundary $\partial D$ only. However, at this moment we shall restrict our proofs only to those which are important to our purposes. We notice first that for sufficiently small $\varepsilon$, depending on dist $(z, f(\partial D))$, the integral (8.12) does not depend on $\varepsilon$. Indeed, the normalizing condition b) implies that $\varrho_{z}^{\varepsilon}(y)-\varrho_{z}^{v}(y)$ is a differential of a smooth $(n-1)$ form with small support contained in the neighbourhood of the point $z$ : this is immcdiately seen in local polar coordinates

$$
\varrho_{z}^{\varepsilon}(y)-\varrho_{z}^{v}(y)=d\left(\int_{0}^{r}\left(\varphi^{\varepsilon}(\tau)-\varphi^{v}(\tau)\right) \tau^{n-1} d \tau d \Theta\right) \equiv d \omega,
$$

where $r=|y-z|$ and $d \Theta$ is the volume (n-1)-form of the unit sphere in $R^{n}$. Obviously the $(n-1)$-form $\omega$ is smooth and compactly supported in $D$. Therefore $\int_{D} f^{*} d \omega=\int_{D} d\left(f^{*} \omega\right)=0$ by Stokes's formula and $\int_{D} f^{*} \varrho_{z}^{\varepsilon}=\int_{D} f^{*} \varrho_{z}^{v}$ as asserted. Thus for each $z \in C_{D}$ we can discard the lim in the formula (8.12). Consequently we have: 
Corollary 8.1. The topological index $\mu(z ; f, D)$ is a continuous function of $z \in C_{D}$.

Theorem 8.6. Let $f$ be a quasiregular mapping of class $W_{p, l o c}^{1}(\Omega), p>n, D \subset \subset \Omega$ and $u(z)$ a bounded measurable function with support contaired in $\boldsymbol{R}^{n} \backslash f(\partial D)$. Then

$$
\int_{D} u(f(x)) J_{f}(x) d x=\int_{R^{n}} u(z) \mu(z ; f, D) d z
$$

Proof. Set $K=\operatorname{supp} u$. Then $K \cap f(\partial D)=\emptyset$ and there exists an $\varepsilon_{0}>0$ such that

By definition

$$
|f(x)-z| \geqq \varepsilon_{0}>0 \text { for } x \in \partial D, \quad z \in K .
$$

$$
\mu(z ; f, D)=\int_{D} \varphi^{\varepsilon}(|f(x)-z|) J_{f}(x) d x \text { for } \varepsilon<\varepsilon_{0} .
$$

Let $\chi_{D}(x)$ be the characteristic function of $D$. Multiplying (8.12) by $u(z)$ and integrating over $\boldsymbol{R}^{n}$ with respect to $z$ we get

$$
\int_{R^{n}} u(z) \mu(z ; f, D) d z=\int_{R^{n}}\left(\int_{R^{n}} \varphi^{\varepsilon}(|f(x)-z|) J_{f}(x) u(z) \chi_{D}(x) d x\right) d z .
$$

Denote

$$
u_{\varepsilon}(y)=\int_{R^{n}} \varphi^{\varepsilon}(|y-z|) u(z) d z
$$

By Fubini's theorem we have

$$
\int_{R^{n}} u(z) \mu(z ; f, D) d z=\int_{R^{n}} u_{\varepsilon}(f(x)) J_{f}(x) \chi_{D}(x) d x .
$$

By the well-known approximation property of the "mollifiers" $\varphi^{\varepsilon}(|y|)$ we have $u_{\varepsilon}(y) \rightarrow u(y)$ almost everywhere. Since $f$ satisfies the $\mathscr{N}^{-1}$-property, $u_{\varepsilon}(f(x)) \rightarrow$ $u(f(x))$ almost everywhere. Therefore by the Lebesgue convergence theorem we get

as desired

$$
\lim _{\varepsilon \rightarrow 0} \int_{R^{n}} u_{\varepsilon}(f(x)) J_{f}(x) \chi_{D}(x) d x=\int_{D} u(f(x)) J_{f}(x) d x
$$

8.6. Further properties of the topological index. The two forms of the change of variables formula make it possible to identify the two functions $\mu(z ; f, D)$ and $N(z ; f, D)$.

Corolla ry 8.2. For any quasiregular map $f: \Omega \rightarrow \boldsymbol{R}^{n}$ and any open subset $D \subset \subset \Omega$,

$$
\mu(z ; f, D)=N(z ; f, D)
$$

for almost every point $z \notin f(\partial D)$. In particular, $\mu(z ; f, D)$ is constant and integervalued on every component of $C_{D}$. The topological index $\mu(z ; f, D)$ does not depend on the choice of the auxiliary "bump" function $\varphi^{\varepsilon}(r)$. 
Proof. The properties (8.13) and (8.6) imply

$$
\int_{R^{n}} u(z) \mu(z ; f, D) d z=\int_{R^{n}} u(z) N(z ; f, D) d z
$$

for each bounded measurable $u(z)$ with support $\subset \subset \boldsymbol{R}^{n} \backslash f(\partial D)$. Hence (8.14) since the left-hand side of (8.14) is continuous in $z$ and the right-hand side is integervalued. The fact that $\mu(z ; f, D)$ is locally constant and integer valued is obvious. The Banach indicatrix $N(z ; f, D)$ depends on $f$ and $D$ only; hence the last assertion of the corollary. Thus we have shown that the topological index $\mu(z ; f, D)$ is independent of the casual choices inherent in the definition. In fact we also see that it is homotopy invariant.

Corollary 8.3. If $f_{t}, 0 \leqq t \leqq 1$, is a family of maps satisfying the conditions of Definition 8.2 and dopending "in a continuous way" on the parameter $t$, and if, for all $t \in[0,1] z \notin f_{t}(\partial D)$. Then

$$
\mu\left(z ; f_{0}, D\right)=\mu\left(z ; f_{1}, D\right) .
$$

Proof is immediate, since an integer-valued continuous function of $t \in[0,1]$ must be constant.

It is possible to derive an expression for the topological index in terms of the homotopy class of the mappings $f_{t \mid \partial D}$. However, we omit the discussion of this topic since we do not use it here.

Applications of the concept of topological index are based on the simple

Proposition 8.1. If $z$ is $(f ; D)$-admissible and $z \notin f(D)$, then $\mu(z ; f, D)=0$. Equivalently

$$
\mu(z ; f, D) \neq 0 \quad \text { implies } \quad z \in f(D) .
$$

Proof. Our assumptions imply $z \notin \overline{f(D)}$. To compute $\mu(z ; f, D)$ we may use any normalized $n$-form $\varrho$ with support in a small cube with center $z$ and diameter $<n^{-1 / 2} \operatorname{dist}(z, \overline{f(D)})$. Since then $f^{*} \varrho \equiv 0$ in $D$, the proposition follows from (8.14).

In view of Proposition 8.1 the topological index is an important tool, describing the image set $f(D)$ in the discussion of the solvability of the equation $f(x)=z$. In this connection we mention

Proposition 8.2. If the point $z$ belongs to the outer domain of the map $f$ then $\mu(z ; f, D)=0$.

In fact, for $z \rightarrow \infty$ the equation $z=f(x)$ has no solutions $x \in D$ (since by assumption $f$ is bounded in $D$ ).

We shall also need

Proposition 8.3. Let $\left\{D_{i}\right\} i=1,2, \ldots$ be a sequence of disjoint open subsets of $D$. Assume that $D \subset \subset \Omega, z$ is $(f, D)$ admissible and $f^{-1}(z) \cap \Omega \subset \cup_{i} D_{i}$. Then 
the point $z$ is $\left(f, D_{i}\right)$-admissible for each $i$ and the topological index $\mu\left(z ; f, D_{i}\right)$ is non-zero for a finite number of indices $\{i\}$ only and

$$
\mu(z ; f, D)=\sum_{i=1}^{\infty} \mu\left(z ; f, D_{i}\right) .
$$

Proof. We show first that $z \in f\left(D_{i}\right)$ only for a finite number of indices $\{i\}$. Really, if $z=f\left(x_{k}\right), x_{k} \in D_{i_{k}} k=1,2, \ldots$, then any accumulation point $x_{0}$ of the sequence $\left\{x_{k}\right\}$ belongs to $f^{-1}(z)$. But $x_{0}$ does not belong to any one of the open sets $D_{i}$, and this contradicts our assumption. It follows that for a sufficiently small neighbourhood $\omega_{z}$ of the point $z$, the open subset $f^{-1}\left(\omega_{z}\right)$ has a non-empty intersection with an at most finite number of $D_{i}$, say $D_{1}, \ldots, D_{N}$. Let the form $\varrho_{z}$ have the support in $\omega_{z}$. Then

$$
\begin{aligned}
\mu(z ; f, D)= & \int_{D} f^{*}(\varrho)=\int_{D} \varrho(f(x)) J_{f}(x) d x=\int_{\substack { N \\
\begin{subarray}{c}{i=1 \\
D_{i}{ N \\
\begin{subarray} { c } { i = 1 \\
D _ { i } } }\end{subarray}} \varrho(f(x)) J_{f}(x) d x \\
& =\sum_{i=1}^{N} \int_{D_{i}} \varrho(f(x)) J_{f}(x) d x=\sum_{i=1}^{N} \mu\left(z, f, D_{i}\right),
\end{aligned}
$$

since the open subsets $D_{i}$ are disjoint. This ends the proof.

\section{GEOMETRICAL AND ANALYTICAL APPLICATIONS}

\subsection{The inverse mapping.}

Theorem 9.1. Lot $f: \Omega \rightarrow \Omega^{\prime}$ be a K-quasiconformal mapping. Then the inverse map $f^{-1}: \Omega^{\prime} \rightarrow \Omega$ is $K^{n-1}$-quasiconformal.

Proof. Let us first observe that the algebraic inequality $\mid A^{n} \leqq K \operatorname{det} A$ implies $\left|A^{-1}\right|^{n} \leqq K^{n-1} \operatorname{det} A^{-1}$ for an arbitrary invertible matrix $A$. That is why the dilatation of $f^{-1}$ is expected to be equal to $K^{n-1}$. Since the adjugate matrix $\operatorname{adj} A=$ (det $A) A^{-1}$, we have

$$
|\operatorname{adj} A| \leqq(K \operatorname{det} A)^{(n-1) / n} \quad \text { whenever } \quad|A|^{n} \leqq K \operatorname{det} A .
$$

We begin with the integral identity

$$
\int_{\Omega^{\prime}} D^{*} \varphi(y) f^{-1}(y) d y=-\int_{\Omega} \operatorname{adj}^{*} f^{\prime}(x) \varphi(f(x)) d x
$$

holding for every test mapping $\varphi: \Omega^{\prime} \rightarrow \boldsymbol{R}^{n}$ of the class $C_{0}^{\infty}\left(\Omega^{\prime}\right)$. To prove this we use the change of variables formula (8.6) applied to the vector function $u(y)=$ $D^{*} \varphi(y) f^{-1}(y)$, getting

$$
\int_{\Omega^{\prime}} D^{*} \varphi(y) f^{-1}(y) d y=\int_{\Omega}\left(J_{f}(x) D^{*} \varphi(f(x))\right) x d x \quad\left(N(y ; f, \Omega)=\chi_{\Omega^{\prime}}(y)\right) .
$$


Consider the test mapping $\eta(x)=\varphi(f(x)), \eta \in \stackrel{\circ}{W}_{n}^{1}(\Omega)$. Since $D \eta(x)=D \varphi(f(x)) D f(x)$, by the definition of the adjugate matrix $\operatorname{adj} f(x)$ we have the formula

Therefore

$$
D \eta(x) \operatorname{adj} f(x)=J_{f}(x) D \varphi(f(x)) \text { a.e. in } \Omega .
$$

$$
\int_{\Omega^{\prime}} D^{*} \varphi(y) f^{-1}(y) d y=\int_{\Omega}\left(\operatorname{adj}^{*} f(x) D^{*} \eta(x)\right) x d x .
$$

The differentiation rule yields $\left(D^{*} \eta(x)\right) x=-\eta(x)+\nabla\langle x, \eta(x)\rangle$, where $\langle x, \eta(x)\rangle$ is the inner product of the vectors $x$ and $\eta(x)$. It is clear that the function $\Psi(x)=$ $\langle x, \eta(x)\rangle$ belongs to $\stackrel{\circ}{W}_{n}^{1}(\Omega)$. Now we appeal to Lemma 1.9, which says that the columns of the matrix adj $f(x)$ are divergence free. The weak form of this fact can be written as follows:

$$
\int_{\Omega} \operatorname{adj}^{*} f(x) \nabla \Psi(x) d x=0
$$

for each function $\Psi$ from $\stackrel{\circ}{W}_{n}^{1}(\Omega)$. In particular, we get the equality

$$
\int_{\Omega^{\prime}} D^{*} \varphi(y) f^{-1}(y) d y=-\int_{\Omega} \operatorname{adj}^{*} f(x) \eta(x) d x,
$$

which is the same as (9.2).

Now by (9.1) and by the Hölder inequality we obtain

$$
\begin{gathered}
\left|\int_{\Omega^{\prime}} D^{*} \varphi(y) f^{-1}(y) d y\right| \leqq K^{(n-1) / n} \int_{\Omega} J_{f}(x)^{(n-1) / n}|\varphi(f(x))| d x \\
\leqq K^{(n-1) / n}\left(\int_{\Omega} J_{f}(x)|\varphi(f(x))|^{\mid n /(n-1)} d x\right)^{(n-1) / n}|\Omega|^{1 / n} \\
=K^{(n-1) / n}\left(\int_{\Omega^{\prime}}|\varphi(y)|^{\mid n /(n-1)} d y\right)^{(n-1) / n}|\Omega|^{1 / n} .
\end{gathered}
$$

Here we have repeatedly used the change of variables formula (8.6). According to the remarks of Section 1 this inequality shows that the map $f^{-1}$ belongs to $W_{n}^{1}\left(\Omega^{\prime}\right)$ and

$$
\left(\int_{\Omega^{\prime}}\left|D f^{-1}(y)\right|^{n} d y\right)^{1 / n} \leqq K^{(n-1) / n}|\Omega|^{1 / n} .
$$

As the map $f^{-1}$ fulfils the condition $\mathscr{N}$, because $f$ satisfies the condition $\mathscr{N}^{-1}$, Theorem 8.3 (applied to the mapping $f^{-1}$ ) yields

$$
|\Omega|=\int_{\Omega^{\prime}} J_{f^{-1}}(y) d y \quad\left(N\left(x ; f^{-1}, \Omega^{\prime}\right)=\chi_{\Omega}\right) .
$$

Therefore

$$
\int_{\Omega^{\prime}}\left|D f^{-1}(y)\right|^{n} d y \leqq K^{n-1} \int_{\Omega^{\prime}} J_{f^{-1}}(y) d y
$$

This inequality may be written with $\Omega^{\prime}$ replaced by an arbitrary subdomain because we could at the beginning restrict our considerations to an arbitrary subdomain 
of $\Omega$. In other words, we are justified in cancelling the integrals on both sides of (9.5) and writing

$$
\left|D f^{-1}(y)\right|^{n} \leqq K^{n-1} J_{f^{-1}}(y)
$$

for almost every $y \in \Omega^{\prime}$. This shows that $f^{-1}$ is a $K^{n-1}$-quasiconformal mapping.

Remark. To write the formula (9.4) we need to know that the inverse map $f^{-1}$ is differentiable almost everywhere in $\Omega^{\prime}$. Since

$$
f(x)-f\left(x_{0}\right)=D f\left(x_{0}\right)\left(x-x_{0}\right)+o\left(\left|x-x_{0}\right|\right), \quad J_{f}\left(x_{0}\right)>0 \quad \text { for } \quad \text { a. e. } \quad x_{0} \in \Omega,
$$

in view of the $\mathscr{N}^{-1}$ property of $f$ we immediately obtain

$$
f^{-1}(y)-f^{-1}\left(y_{0}\right)=\left(D f\left(x_{0}\right)\right)^{-1}\left(y-y_{0}\right)+o\left(\left|y-y_{0}\right|\right)
$$

for almost all $y_{0}=f\left(x_{0}\right) \in \Omega^{\prime}$.

\subsection{Isomorphisms of Sobolev spaces $W_{n}^{1}$.}

Corollary 9.1. Let $f: \Omega \rightarrow \Omega^{\prime}$ be a quasiconformal mapping such that $f \in W_{n}^{1}(\Omega),|D f(x)|^{n} \leqq K J_{f}(x)$. Let $v$ be a function from the Sobolev space $W_{n}^{1}\left(\Omega^{\prime}\right)$. Then the function $u(x)=v(f(x))$ belongs to $W_{n}^{1}(\Omega)$ and

$$
\int_{\Omega}|\nabla u(x)|^{n} d x \leqq K \int_{\Omega^{\prime}}|\nabla v(y)|^{n} d y .
$$

In particular, the linear operator $f_{*}: \stackrel{\circ}{W}_{n}^{1}\left(\Omega^{\prime}\right) \rightarrow \stackrel{\circ}{W}_{n}^{1}(\Omega)$ defined by $\left(f_{*} v\right)(x)=v(f(x))$ is an isomorphism the norm of which does not exceed $\sqrt[n]{K}$. Here the norm in $\stackrel{\circ}{W}_{n}^{1}(\Omega)$ is given by $\|u\|=\left(\int_{\Omega}|\nabla u|^{n}\right)^{1 / n}$.

Proof. If $v \in C^{1}\left(\Omega^{\prime}\right)$, then obviously $u \in W_{n}^{1}(\Omega)$ and $\nabla u(x)=D^{*} f(x) \nabla v(f(x))$. Hence $|\nabla u(x)|^{n} \leqq|D f(x)|^{n}|\nabla v(f(x))|^{n} \leqq K|\nabla v(f(x))|^{n} J_{f}(x)$. Since $\nabla u \in W_{n}^{1}\left(\Omega^{\prime}\right)$, we get by Theorem 8.4 that $|\nabla v(f(x))|^{n} J_{f}(x)$ is integrable on $\Omega$ and

$$
\int_{\Omega}|\nabla u(x)|^{n} \leqq K \int_{\Omega^{\prime}}|\nabla v(y)|^{n} d y .
$$

This may be simply generalized for an arbitrary $v$ from $W_{n}^{1}\left(\Omega^{\prime}\right)$ by an obvious approximation. Let us remark that the inverse operator $\left(f_{*}\right)^{-1}: \stackrel{\circ}{W}_{n}^{1}(\Omega) \rightarrow \stackrel{\circ}{W}_{n}^{1}\left(\Omega^{\prime}\right)$ is equal to $\left(f^{-1}\right)_{*}$ with the inverse map $f^{-1}: \Omega^{\prime} \rightarrow \Omega$.

Another direct consequence of the above results is the important observation that the conformal capacity is $K$-invariant under $K$-quasiconformal mappings. This means:

Corollary 9.2. Let $F$ be a compact subset of a domain $\Omega$ and let $f: \Omega \rightarrow \Omega^{\prime}$ be a K-quasiconformal mapping transforming $F$ onto a compact set $F^{\prime}=f(F)$. Then

$$
\mathrm{Cap}_{n}(F, \Omega) \leqq K \operatorname{Cap}_{n}\left(F^{\prime}, \Omega^{\prime}\right) .
$$


In fact, by (9.6) and by the definition of conformal capacity we have

$$
\begin{gathered}
K \operatorname{Cap}_{n}\left(F^{\prime}, \Omega^{\prime}\right)=\inf _{\substack{v \in W_{n}^{1}\left(\Omega^{\prime}\right) \\
v \geqq 1}} K \int_{\Omega^{\prime}}|\nabla v(y)|^{n} d y \geqq \inf _{\substack{u \in W_{n}^{1}(\Omega) \\
u \geqq 1}} \int_{\substack{n \\
\text { on }}}|\nabla u(x)|^{n} d x \\
\geqq \operatorname{Cap}_{n}(F, \Omega) .
\end{gathered}
$$

9.3. Local topological properties of quasiregular mappings. Now we have all the tools necessary to prove the fundamental fact that a quasiregular mapping is discrete and open. We recall that a map $f: \Omega \rightarrow \boldsymbol{R}^{n}$ is discrete if for each $x_{0} \in \Omega$ there exists a neighbourhood $\Omega_{x_{0}}$ of $x_{0}$ in $\Omega$ such that $f(x) \neq f\left(x_{0}\right)$ for $x \in \Omega_{x_{0}}$ $\left\{x_{0}\right\}$. The map $f$ is open in $\Omega$ if the image set $f(D)$ is open in $\boldsymbol{R}^{\boldsymbol{n}}$ whenever $D$ is an open subset of $\Omega$. These properties of q.r. mappings were first proved by Ju. G. Rešetnjak.

We start with the following lemma.

Lemma 9.1. Let $f: \Omega \rightarrow \boldsymbol{R}^{n}$ be an orientation preserving map of class $W_{p}^{1}(\Omega)$, $p>n, J_{f}(x)>0$ for almost every $x \in \Omega$. Let $z \in f(D)-f(\partial D)$ for some subdomain $D \subset \simeq \Omega$. Then

$$
\mu(z ; f, D) \geqq 1 \text {. }
$$

Proof. Take as the density $\varrho(y)$ in the definition of $\mu(z ; f, D)$ a smooth $n$-form $\varrho(y) d y$ such that $\varrho(y)>0$ in a small neighbourhood of the point $z \in f(D)-$ $f(\partial D)$. Then

$$
\mu(z ; f, D)=\int_{D} \varrho(f(x)) J_{f}(x) d x>0
$$

since $\varrho(f(x)) J_{f}(x)>0$ on a subset of positive measure $(\varrho \circ f$ is continuous and $\varrho(z)>0)$.

Now $\mu(z ; f, D)$ assumes only integer values. Thus we get

as needed.

$$
\mu(z ; f, D) \geqq 1
$$

Proposition 9.1. Let $f$ satisfy the assumptions of Lemma 9.1. If $N(z, f, D)<$ $+\infty$ for a point $z \in f(D)-f(\partial D)$, then

$$
1 \leqq N(z, f, D) \leqq \mu(z ; f, D) .
$$

Proof. By assumption $z=f\left(x_{i}\right)$ only for a finite number of points $x_{i} \in D$, $i=1,2, \ldots, k$. For $i=1, \ldots, k$ let $D_{i}$ be a small ball with centre $x_{i}, D_{i} \subset D$, such that $D_{i} \cap D_{j}=\emptyset$ for $i \neq j$. Then $z \notin f\left(\partial D_{i}\right)$ for $i=1, \ldots, k$. Moreover,

$$
1 \leqq N(z, f, D)=\sum_{i=1}^{k} N\left(z, f, D_{i}\right) \leqq \sum_{i=1}^{k} \mu\left(z ; f, D_{i}\right) \leqq \mu(z ; f, D)
$$


since $N\left(z, f, D_{i}\right)=1 \leqq \mu\left(z ; f, D_{i}\right)$ in view of Lemma 9.1. Notice that the condition $N(z, f, D)<+\infty$ assures that Lemma 9.1 is applicable. We also used Proposition 8.3. In contrast to (8.14) the inequality (9.9) holds for each admissible $z$.

Now we introduce a convenient condition $L$.

Condition $L$. A sense preserving $\left(J_{f}(x) \geqq 0\right.$ a.e. in $\left.\Omega\right)$ mapping $f \in W_{p}^{1}(\Omega), p>n$, will be said to satisfy the condition $L$ if for each point $x \in \Omega$ there exists an arbitrary small spherical neighbourhood $B(x, r)$ of $x, B(x, r) \subset \Omega$ such that

$$
f(x) \notin f(\partial B(x, r)) .
$$

The neighbourhoods $B(x, r)$ satisfying (9.10) will be called quasinormal.

Proposition 9.2. If the mapping $f: \Omega \rightarrow \boldsymbol{R}^{n}, f \in W_{p}^{1}(\Omega), p>n$, satisfies the cordition $L$, then for every domain $D \subset \subset \Omega$ and any $z \in f(D)-f(\partial D)$ the inequality (9.9) holds.

Proof. Set $k=\mu(z ; f, D)$ and assume that $(9.9)$ is not true. Let $x_{1}, \ldots, x_{k+1}$ be $k+1$ distinct points in $f^{-1}(z) \cap D$. Let $B\left(x_{i}, r_{i}\right), i=1,2, \ldots, k+1$, be a sequence of disjoint quasinormal neighbourhoods. Then, as in the proof of Proposition 9.1,

$$
k+1 \leqq \sum_{i=1}^{k+1} \mu\left(z ; f, B\left(x_{i}, r_{i}\right)\right) \leqq \mu(z ; f, D)=k,
$$

which is a contradiction.

Proposition 9.3. Let $f$ be as in Proposition 9.2. Then for each point $x_{0} \in \Omega$ there exists a neighbourhood $\omega_{x_{0}} \subset \Omega$ such that

a) $f(x) \neq f\left(x_{0}\right)$ for $x \in \omega_{x_{0}}$ and $x \neq x_{0}$,

b) $f\left(\omega_{x_{0}}\right)$ is an open set.

Proof. Applying Proposition 9.2 to a quasinormal neighbourhood $B\left(x_{0}, r_{0}\right)$ of $x_{0}$ we conclude that in $B\left(x_{0}, r_{0}\right)$ there exists a finite number $\left(\leqq \mu\left(f\left(x_{0}\right) ; f, B\left(x_{0}, r_{0}\right)\right)\right)$ of points $x_{k}, k=1, \ldots, N, f\left(x_{k}\right)=f\left(x_{0}\right)$. Then any ball $B\left(x_{0}, r\right)$ with $r<\min \left\{\left|x_{k}-x_{0}\right|\right.$; $k=1, \ldots, N\}$ satisfies the condition a). Since $\mu\left(f\left(x_{0}\right) ; f, B\left(x_{0}, r\right)\right) \geqq 1$, it follows that $\mu\left(z ; f, B\left(x_{0}, r\right)\right) \geqq 1$ for $z$ sufficiently close to $f\left(x_{0}\right)$. Proposition 8.1 implies then that $z \in f\left(B\left(x_{0}, r\right)\right) \subset f(D)$. Thus we see that a sufficiently small ball with centre $f\left(x_{0}\right)$ is contained in $f(D)$. This proves b).

Proposition 9.3 expresses the fundamental fact that the condition $L$ for a mapping $f: \Omega \rightarrow \boldsymbol{R}^{n}$ of class $W_{p}^{1}(\Omega), p>n$, implies that the mapping $f$ is discrete and open. Actually the assumption that $f \in W_{p}^{1}(\Omega)$ is superfluous; however, in quasiconformal theory this weaker version is sufficient. Conversely, the conditions a) and b) of Proposition 9.3 imply the condition $L$. The equivalence of the condition $L$ and the conditions a) and b) for continuous orientation preserving mappings of topological manifolds was proved in the paper of Titus-Young in 1962 [37]. 
Lemma 9.2. Let $A$ be a closed set in $\boldsymbol{R}^{n}$. Then for every $x \in \boldsymbol{R}^{n}$ the outer Hausdorff measure $\gamma_{1}(A)$ can be estimated from below by

$$
\gamma_{1}(A) \geqq \frac{1}{\sqrt{n}} \gamma_{1}\{r>0 ; S(x, r) \cap A \neq 0\} .
$$

In particular, if $\gamma_{1}(A)=0$, then for each $x \in \boldsymbol{R}^{n}$ we can find a ball $B(x, r)$ of arbitrary small radius $r$ such that $A \cap \partial B(x, r)=\emptyset$.

The last statement expresses the fact that the topological dimension of the set $A$ is zero.

Proof. Let $\mathscr{F}$ be an arbitrary countable family of cubes covering the set $A$. If $S(x, r) \cap A \neq \emptyset$, then $S(x, r)$ intersects at least one of the cubes $Q \in \mathscr{F}$. Therefore the set of parameters $r$ defined by $\{r>0 ; S(x, r) \cap A \neq \emptyset\}$ is covered by a family of intervals of length diam $(Q)$, where $Q \in \mathscr{F}$. Thus

$$
\gamma_{1}(A)=\inf _{\mathscr{F}} \sum_{Q \in \mathscr{F}}|Q|^{1 / n}=\frac{1}{\sqrt{n}} \inf _{\mathscr{F}} \sum_{Q \in \mathscr{F}} \operatorname{diam} Q \geqq \frac{1}{\sqrt{n}} \gamma_{1}\{r>0 ; S(x, r) \cap A \neq \emptyset\}
$$

as assertcd.

As an immediate consequence of this lemma we get

Lemma 9.3. Let $f: \Omega \rightarrow \boldsymbol{R}^{n}$ be a quasiregular mapping, not reducing to a constant map on any component of $\Omega$. Then $f$ satisfies the condition $L$.

Finally in view of Proposition 9.3 we conclude:

Theorem 9.2. Every quasiregular mapping $f: \Omega \rightarrow \boldsymbol{R}^{n}, f \not$ constant, is discrete and open.

\subsection{Measure of $B_{f}$ and $f\left(B_{f}\right)$.}

Theorem 9.3. For any quasiregular map $f: \Omega \rightarrow \boldsymbol{R}^{n}, f \not \equiv$ constant, mes $B_{f}=0$. Consequently also mes $f\left(B_{f}\right)=0$.

The proof is based on two lemmas.

Lemma 9.4. Let $f: \Omega \rightarrow \boldsymbol{R}^{n}$ be a mapping of class $W_{p}^{1}(\Omega), p>n, J_{f}(x) \geqq 0$ a.e. Let $x_{0} \in \Omega$ be a point of differentiability of $f$ and a Lebesgue point for the Jacobian, $J_{f}\left(x_{0}\right)>0$. Then there exists a neighbourhood $D$ of $x_{0}$ such that the point $z=f\left(x_{0}\right) \in f(D)-f(\partial D)$ and

$$
\mu(z ; f, D)=1 \text {. }
$$

Proof. We may assume without loss of generality that $x_{0}=0, z=f\left(x_{0}\right)=0$, $D f\left(x_{0}\right)=I-$ the unit matrix. Since $f$ is differentiable at the point $x_{0}=0$, then the expansion

holds, where $v(x)=o(|x|)$.

$$
f(x)=x+v(x)
$$


We take for $D$ a ball $B(0, r)$ with $r$ small enough to satisfy $|f(x)| \geqq(1 / 2)|x|$ for $|x| \leqq r$. The point $z=f\left(x_{0}\right)=0$ is then $(f, D)$-admissible and

$$
\begin{aligned}
\mu(z ; f, D)= & \lim _{\varepsilon \rightarrow 0} \int_{B(0, r)} Q^{\varepsilon}(f(x)) J_{f}(x) d x=\lim _{\varepsilon \rightarrow 0} \int_{B(0, r)} \varrho^{\varepsilon}(f(x)) d x \\
& +\lim _{\varepsilon \rightarrow 0} \int_{B(0, r)} \varrho^{\varepsilon}(f(x))\left(J_{f}(x)-1\right) d x,
\end{aligned}
$$

where $\varrho^{\varepsilon}(y)=\varepsilon^{-n} \Psi\left(\varepsilon^{-1}|y|\right), \Psi \in C^{\infty}\left(\boldsymbol{R}^{1}\right), \Psi(t)=0$ for $t \geqq 1, \int \Psi(|y|) d y=1$. The first limit is exactly equal to 1 . In fact, for $\varepsilon \equiv r$, supp $\varrho^{\varepsilon}(f(x)) \subset B(0,2 \varepsilon)$ and

$$
\begin{gathered}
\lim _{\varepsilon \rightarrow 0} \int_{B(0, r)} \varrho^{\varepsilon}(f(x)) d x=\lim _{\varepsilon \rightarrow 0} \varepsilon^{-n} \int_{B(0,2)} \Psi\left(\mid \varepsilon^{-1} x+\varepsilon^{-1} v(x)\right) d x \\
=\lim _{\varepsilon \rightarrow 0} \int_{B(0,2)} \Psi\left(\left|y+\varepsilon^{-1} v(\varepsilon y)\right|\right) d y=\int_{B(0,2)} \Psi(|y|) d y=1 .
\end{gathered}
$$

To estimate the second limit on the right hand side of (9.12) we use the fact that $x_{0}=0$ is the Lebesgue point of the Jacobian and $J_{f}\left(x_{0}\right)=1$,

$$
\begin{aligned}
& \left|\int_{B(0, r)} \varrho^{\varepsilon}(f(x))\left(J_{f}(x)-1\right) d x\right| \leqq \varepsilon^{-n} \int_{B(0,2 \varepsilon)}\left|\Psi\left(\left|\varepsilon^{-1} x+\varepsilon^{-1} v(x)\right|\right)\right|\left|J_{f}(x)-1\right| d x \\
& \leqq C(n) \underset{B(0,2 \varepsilon)}{f}\left|J_{f}(x)-1\right| d x \rightarrow 0 \quad \text { as } \quad \varepsilon \rightarrow 0 .
\end{aligned}
$$

This completes the proof of the lemma.

Lemma 9.5. Let $f: \Omega \rightarrow \boldsymbol{R}^{n}$ be a quasiregular mapping and let $x_{0}$ be a point of differentiability of $f$ ard the Lebesgue point for the Jacobian, $J_{f}\left(x_{0}\right)>0$. Then $f$ is a local homeomorphism at $x_{0}$.

Proof. In view of the previous lemma for sufficiently small quasinormal neighbourhoods $B\left(x_{0}, r\right)$ of $x_{0}$, the topological index

$$
\mu\left(z ; f, B\left(x_{0}, r\right)\right)=1 \text { for } z \in f\left(B\left(x_{0}, r\right)\right)-f\left(\partial B\left(x_{0}, r\right)\right) .
$$

By Lemma 9.3, $f$ satisfies the condition $L$ and we are justified in using Proposition 9.2 getting

$$
1 \leqq N\left(z, f, B\left(x_{0}, r\right)\right) \leqq \mu\left(z ; f, B\left(x_{0}, r\right)\right)=1 .
$$

In other words, the map $f$ is $1-1$ on the set $f^{-1}\left(f\left(B\left(x_{0}, r\right)\right) \backslash f\left(\partial B\left(x_{0}, r\right)\right)\right) \cap B\left(x_{0}, r\right)$, which is open because $f\left(B\left(x_{0}, r\right)\right)-f\left(\partial B\left(x_{0}, r\right)\right)$ is open, and contains the point $x_{0}$ because $B\left(x_{0}, r\right)$ is a quasinormal neighbourhood of $x_{0}$. Since the map $f$ is open, the inverse map $f^{-1}$ is continuous. This shows that $f$ is a local homeomorphism at $x_{0}$. Finally, Theorem 9.3 follows from Lemma 9.5, which is applicable for almost all points $x_{0} \in \Omega$, giving mes $B_{f}=0$; the assertion that $\operatorname{mes} f\left(B_{f}\right)=0$ is a consequence of the $\mathscr{N}$ property of $f$. 
9.5. Composition of quasiregular mappings. The important question about the composition of quasiregular mappings is somewhat delicate in the set-up of our analytical Definition 2.1. It naturally splits into two questions corresponding to the conditions a) and b) of the definition in Section 2.

Lemma 9.6. Let $\varphi \in W_{n, \mathrm{loc}}^{1}\left(\Omega^{\prime}\right)$ and let $f: \Omega \rightarrow \Omega^{\prime}$ be a quasiregular mapping of a domain $\Omega$ into $\Omega^{\prime}$. Then $f_{*} \varphi=\varphi \circ f \in W_{n, 1 \mathrm{oc}}^{1}(\Omega)$. Moreover, for almost every $x \in \Omega$

$$
\frac{\partial\left(f_{*} \varphi\right)}{\partial x^{i}}(x)=\sum_{k=1}^{n} \frac{\partial \varphi}{\partial y^{k}}(f(x)) \frac{\partial f^{k}}{\partial x^{i}}, \quad i=1,2, \ldots, n
$$

i.e., the usual chain rule for differentiation of the composite function holds.

Proof. Let $x_{0} \in \Omega$ be an arbitrary point and let $B=B\left(x_{0}, r\right)$ be a quasinormal neighbourhood of $x_{0}, f\left(x_{0}\right) \in f(B)-f(\partial B)$. Let $D \subset \boldsymbol{R}^{n}-f(\partial B)$ be the component containing $f\left(x_{0}\right)$ and let $\varphi_{h}(y), h=1,2, \ldots$ be a sequence of $C_{0}^{\infty}(D)$ functions bounded in $W_{n}^{1}\left(\boldsymbol{R}^{n}\right)$ and approximating $\varphi$ in the sense of $W_{n}^{1}(U)$ for an open $U$ such that $f\left(x_{0}\right) \in U \subset \subset D$. Then $V=f^{-1}(U) \cap B$ is a neighbourhood of $x_{0}$. The functions $\varphi_{h}(f(x))$ obviously belong to $W_{n}^{1}(\Omega)$; the formula

$$
\frac{\partial\left(f_{*} \varphi_{h}\right)}{\partial x^{i}}(x)=\sum_{k=1}^{n} \frac{\partial \varphi_{h}}{\partial y^{k}}(f(x)) \frac{\partial f^{k}}{\partial x^{i}} \quad i=1,2, \ldots
$$

holds for almost every $x \in \Omega$, and $\left(\varphi_{h} \circ f\right)(x) \rightarrow(\varphi \circ f)(x)$ for almost every $x \in V$. By the well-known property of Sobolev spaces it is enough to show that the integrals

$$
\int_{V}\left|\frac{\partial\left(\varphi_{h} \circ f\right)(x)}{\partial x^{i}}\right|^{n} d x \quad i=1,2, \ldots, n
$$

are bounded uniformly in $h$. The quasiregularity of $f$ implies

$$
\left|\frac{\partial\left(\varphi_{h} \circ f\right)(x)}{\partial x^{i}}\right|^{n} \leqq K\left|\left(\nabla \varphi_{h}\right)(f(x))\right|^{n} J_{f}(x)
$$

for almost every $x \in \Omega$.

Since $\nabla \varphi_{h} \in L^{\infty}\left(\boldsymbol{R}^{n}\right)$, supp $\nabla \varphi_{h} \subset D \subset \boldsymbol{R}^{n}-f(\partial B)$, we are justified in using the change of variables formula (8.13)

$$
\int_{B}\left|\frac{\partial\left(\varphi_{h} \circ f\right)(x)}{\partial x^{i}}\right|^{n} d x \leqq K \int_{R^{n}}\left|\nabla \varphi_{h}(y)\right|^{n} \mu(y ; f, B) d y=K \int_{D}\left|\nabla \varphi_{h}(y)\right|^{n} \mu(y ; f, B) d y .
$$

Since $D$ is the $f\left(x_{0}\right)$-component of $\boldsymbol{R}^{n}-f(\partial B)$, then $\mu(y ; f, B)=\mu\left(f\left(x_{0}\right) ; f, B\right)$ for all $y \in D$. The integrals $\int_{D}\left|\nabla \varphi_{h}(y)\right|^{n} d y$ are bounded uniformly in $h$, say 
by $M$. Hence

$$
\int_{V}\left|\frac{\partial\left(\varphi_{h} \circ f\right)(x)}{\partial x^{i}}\right|^{n} d x \leqq K \mu\left(f\left(x_{0}\right) ; f, B\right) \cdot M
$$

and the required uniform estimate follows.

Now we can prove

Theorem 9.4. If $f: \Omega \rightarrow \Omega^{\prime} \subset \boldsymbol{R}^{n}$, and $g: \Omega^{\prime} \rightarrow \boldsymbol{R}^{n}$ are $K_{1}$ and $K_{2}$-quasiregular mappings, respectively, then the composition $g \circ f: \Omega \rightarrow \boldsymbol{R}^{n}$ is $K_{1} \cdot K_{2}$-quasiregular.

Proof. In view of Lemma 9.6 we have to prove only that the condition b) of the definition is fulfilled with the dilatation constant estimated by $K_{1} \cdot K_{2}$. The chain rule of Lemma 9.6 gives the formulas

$$
D(g \circ f)=D g \circ D f \quad \text { and } \quad J_{g \circ f}(x)=J_{g}(f(x)) \cdot J_{f}(x) .
$$

We need to prove the inequality

$$
|D(g \circ f)(x)|^{n} \leqq K_{1} \cdot K_{2} J_{g \circ f}(x)
$$

for almost every $x \in \Omega$.

This follows if we multiply both sides of the inequalities

and

$$
|D f(x)|^{n} \leqq K_{1} J_{f}(x)
$$

$$
|D g(f(x))|^{n} \leqq K_{2} J_{g}(f(x))
$$

valid for almost every $x \in \Omega$. The first is clear and the second holds, for almost every $x \in \Omega$, since the map $f$ satisfies the condition $\mathscr{N}^{-1}$.

\section{References}

[1] Ahlfors, L.: Conditions for quasiconformal deformations in several variables. - Contributions to analysis, edited by L. V. Ahlfors et al., Academic Press, New York-London, 1974, 19-25.

[2] Banach, S.: Sur les lignes rectifiables et les surfaces dont l'arie est finie. - Fund. Math. 7, $1925,225-237$.

[3] Bers, L.: Quasiconformal mappings, with applications to differential equations, function theory and topology. - Bull Amer. Math. Soc. 83: 6, 1977, 1083-1100.

[4] BoJARSKĬ, B. V. (Боя рский, Б. В.) : Гомеоморфные решения систем Бельтрами. - Dokl. Akad. Nauk SSSR 102, 1955, 661-664.

[5] BojARSKIĬ, B. V.: Generalized solutions of a system of differential equations of first order and elliptic type with discontinuous coefficients. - Math. Sb. N. S. 43 (85), 1957, 451-503 (Russian).

[6] Bojarskil̆, B.: Nonlinear overdetermined systems of equations and infinitesimal deformations of quasiconformal mappings. - Proceedings of an All-Union Conference on Partial Differential Equations, Moscow State Univ., Moscow, 1976, Moskov. GOS. Univ., Meh.-Mat. Fakul'tet, Moscow, 1978, 53-56 (Russian). 
[7] Bojarskiř, B., and T. IwANIEC: Topics in quasiconformal theory in several variables. - Proceedings of the First Finnish-Polish Summer School in Complex Analysis, Podlesice, 1977, Part II, Univ. Łódź, Łódź, 1978, 21-214.

[8] Bojarskiľ, B., and T. Iwaniec: Some new concepts in the analytical theory of QC-maps in $R^{n}, n \geqq 3$, and differential geometry. - The Corference on Global Analysis, Garwitz, DDR, October 1981 (not published).

[9] Bojarskiľ, B., and T. IwANIEC: Another approach to Liouville theorem. - Math. Nachr. 107, 1982, 253-262.

[10] Bojarskil̆, B., and T. IwANIEC: Analytical methods in quasiconformal mapping theory. In preparation.

[11] Calderon, A. P.: On differentiability of absolute continuous functions. - Riv. Mat. Univ. Parma 2, 1951, 203-213.

[12] Elcrat, A., and N. G. Meyers: Some results on regularity for solutions of non-linear elliptic systems and quasiregular functions. - Duke Math. J. 42, 1975, 121-136.

[13] Gehring, F. W.: Rings and quasiconformal mappings in space. - Trans. Amer. Math. Soc. 103, $1962,353-393$.

[14] Gehring, F. W.: The $L^{p}$-integrability of the partial derivatives of a quasiconformal mapping. Acta Math. 130, 1973, 265-277.

[15] Giaquinta, M.: Multiple integrals in the calculus of variations and non-linear elliptic systems. Sonderforschungsbereich 72, preprint no. 443, Bonn, 1981.

[16] Granlund, S., P. Lindqvist, and O. Martio: Conformally invariant variational integrals. Trans. Amer. Math. Soc. 277, 1983, 43-73.

[17] HeINZ, E.: An elementary analytic theory of the degree of mapping in $n$-dimensional space. J. Math. Mech. 8, 1959, 231-247.

[18] Ivanov, L. D.: Variations of sets and functions. - Izdat. "Nauka", Moscow, 1975 (Russian).

[19] IwANIEC, T.: The $L^{p}$-integrability of the derivatives of quasiconformal mappings and their generalizations. - Sonderforschungsbereich 72, preprint no. 276, Bonn, 1979.

[20] IwANIEC, T.: Regularity theorems for solutions of partial differential equations for quasiconformal mappings in several dimensions. - Pol. Acad. Sci., preprint no. 153, 1978 and printed in Dissertationes Mathematicae CXCVIII, Warsaw, 1982.

[21] IwANIEC, T.: On $L^{p}$-integrability in PDE's and quasiregular mappings for large exponents. Ann. Acad. Sci. Fenn. Ser. A I Math. 7, 1982, 301-322.

[22] Iwaniec, T.: Projections onto gradient fields and $L^{p}$-estimates for degenerated elliptic operators. - Studia Math. T. LXXV. 1983, 293-312.

[23] Lavrentiev, M.: Sur une classe de représentations continues. - Rec. math. Moscou 42, 1935, $407-423$.

[24] Lehto, O., and K. I. ViRTANen: Quasikonforme Abbildungen. - Springer-Verlag, BerlinHeidelberg-New York, 1965.

[25] Martio, O.: On the integrability of the derivatives of a quasiregular mapping. - Math. Scand. $35,1974,43-48$.

[26] Martio, O., S. Rickman, and J. VÄISÄLÄ: Definitions for quasiregular mappings. - Ann. Acad. Sci. Fenn. Ser. A I Math. 448, 1969, 1-40.

[27] Martio, O., S. Rickman, and J. VÄISÄLÄ: Distortion and singularities of quasiregular mappings. - Ann. Acad. Sci. Fenn. Ser. A I Math. 465, 1970, 1-13.

[28] Martio, O., S. RickMAN, and J. VÄIIÄLÄ: Topological and metric properties of quasiregular mappings. - Ann. Acad. Sci. Fenn. Ser. A I Math. 488, 1971, 1-31.

[29] ReŠEtNJAK, JU. G.: Spatial mappings with bounded distortion. Sibirsk. Mat. Ž. 8, 1967, 629-658 (Russian).

[30] ReŠEtNJAK, Ju. G.: The set of singular points of solutions of certain nonlinear equations of elliptic type. - Sibirsk. Mat. Ž. 9, 1968, 354-367 (Russian). 
[31] REŠETNJAK, JU. G.: Mappings with bounded distortion as extremals of integrals of Dirichlet type. - Sibirsk. Mat. Ž. 9, 1968, 652-666 (Russian).

[32] Rickman, S.: A defect relation for quasimeromorphic mappings. - Ann. of Math. (2) 114, 1981, 165-191.

[33] SAKs, S.: Theory of the integral. - Warsaw, 1937.

[34] Soвolev, S. (Соболев, С.): Об одной теореме функционального анализа. - Mat. Sb. 4, 1938, 471-497.

[35] Sobolev, S. L.: Applications of functional analysis in mathematical physics. - Leningrad, 1950 (Russian). English translation: Translations of Mathematical Monographs, Vol. 7, American Mathematical Society, Providence, Rhode Island, 1963.

[36] Stein, E.: Singular integrals and differentiability properties of functions. - Princeton University Press, Princeton, N. J. 1970.

[37] Titus, C. J., and G. S. Young: The extension of interiority, with some applications. - Trans. Amer. Math. Soc. 103, 1962, 329-340.

[38] VÄISÄLÄ, J.: Lectures on $n$-dimensional quasiconformal mappings. - Lecture Notes in Mathematics 229, Springer-Verlag, Berlin-Heidelberg-New York, 1971.

[39] VÄISÄLÄ, J.: A survey of quasiregular maps in $R^{n}$-Proceedings of the International Congress of Mathematicians, Helsinki 1978, 685-691.

[40] VekUA, I. N.: Generalized analytic functions. - Pergamon Press, London, 1962.

[41] VitušKın, A. G.: On multidimensional variations. - Gosudatstv. Izdat. Tehn.-Teor. Lit., Moscow, 1955 (Russian).

[42] REŠETNJAK, JU. G.: Spatial mappings with bounded distortion. Izdat. Nauka, Moscow, 1982.

[43] REŠETNJAK, JU. G.: Stability theorems in geometry and analysis. Izdat. Nauka, Moscow, 1982.

University of Warsaw

Department of Mathematics

Warsaw

Poland

Received 17 March 1983 